\title{
Partial Gaussian bounds for degenerate differential operators II
}

\author{
A. F. M. TER Elst and El MaAti OuhabaZ
}

\begin{abstract}
Let $A=-\sum \partial_{k} c_{k l} \partial_{l}$ be a degenerate sectorial differential operator with complex bounded mesaurable coefficients. Let $\Omega \subset \mathbb{R}^{d}$ be open and suppose that $A$ is strongly elliptic on $\Omega$. Further, let $\chi \in C_{\mathrm{b}}^{\infty}\left(\mathbb{R}^{d}\right)$ be such that an $\varepsilon$ neighbourhood of $\operatorname{supp} \chi$ is contained in $\Omega$. Let $v \in(0,1]$ and suppose that the $c_{k l \mid \Omega} \in C^{0, v}(\Omega)$. Then we prove (Hölder) Gaussian kernel bounds for the kernel of the operator $u \mapsto \chi S_{t}(\chi u)$, where $S$ is the semigroup generated by $-A$. Moreover, if $v=1$ and the coefficients are real, then we prove Gaussian bounds for the kernel of the operator $u \mapsto \chi S_{t} u$ and for the derivatives in the first variable. Finally we show boundedness on $L_{p}\left(\mathbb{R}^{d}\right)$ of restricted Riesz transforms.
\end{abstract}

Mathematics Subject Classification (2010): 35J70 (primary); 35K08 (secondary).

\section{Introduction}

If $A$ is a strongly elliptic second-order operator on $\mathbb{R}^{d}$ in divergence form with complex bounded Hölder continuous coefficients, then it is well known that it generates a holomorphic semigroup $S$ which satisfies Gaussian kernel bounds and Gaussian bounds for first order derivatives in each of the variables. If $A$ is merely partially strongly elliptic on an open set $\Omega \subset \mathbb{R}^{d}$ then in general Gaussian bounds on $\mathbb{R}^{d}$ fail, but in a previous paper [5] we showed Gaussian kernel bounds on good parts of $\Omega$ if the coefficients of $A$ are real and measurable. Precisely, if $\chi \in C_{\mathrm{b}}^{\infty}(\Omega, \mathbb{R})$ and if $A$ is strongly elliptic on supp $\chi$, then for all $t>0$ the operator $M_{\chi} S_{t} M_{\chi}$ has a Hölder continuous kernel satisfying (Hölder) Gaussian bounds, where $M_{\chi}$ is the multiplication operator with the function $\chi$. In this paper we extend this to (Hölder) derivatives of the kernel if the coefficients of the operator $A$ are complex Hölder continuous on $\Omega$ and the distance $d\left(\operatorname{supp} \chi, \Omega^{\mathrm{c}}\right)>0$, that is an $\varepsilon$-neighbourhood of supp $\chi$ is still in $\Omega$. If in addition the coefficients are in $W^{1, \infty}(\Omega)$ and real on

Most of this work was carried out whilst the first named author visited the University of Bordeaux I and the second named author was visiting the Department of Mathematics at the University of Auckland. Both authors wish to thank the University of Bordeaux I, the University of Auckland and the CNRS for financial support. Part of this work is supported by the Marsden Fund Council from Government funding, administered by the Royal Society of New Zealand.

Received January 11, 2012; accepted November 12, 2012. 
$\mathbb{R}^{d}$, then we also show that for all $t>0$ the operator $M_{\chi} S_{t}$ has a kernel $K_{t}$ satisfying Gaussian bounds. This is remarkable, since there is no cut-off for the operator $M_{\chi} S_{t}$ on the right. Moreover, we show that there exists a representative of the kernel $K_{t}$ such that $(t, x, y) \mapsto K_{t}(x, y)$ is measurable on $(0, \infty) \times \mathbb{R}^{d} \times \mathbb{R}^{d}$ and $x \mapsto K_{t}(x, y)$ is once differentiable for all $y \in \mathbb{R}^{d}$ and $t>0$, and the derivatives satisfy (Hölder) Gaussian bounds. This allows to prove boundedness of the restricted Riesz transform $\nabla M_{\chi}(I+A)^{-1 / 2}$ on $L_{p}\left(\mathbb{R}^{d}\right)$ for all $p \in(1, \infty)$.

Throughout this paper the field is $\mathbb{C}$. Fix $d \in \mathbb{N}$ and for all $k, l \in\{1, \ldots, d\}$ let $c_{k l}: \mathbb{R}^{d} \rightarrow \mathbb{C}$ be a measurable bounded function. Suppose that the matrix $C(x):=\left(c_{k l}(x)\right)$ is uniformly sectorial for all $x \in \mathbb{R}^{d}$, i.e., there exists a $\theta \in\left[0, \frac{\pi}{2}\right)$ such that

$$
\sum_{k, l=1}^{d} c_{k l}(x) \xi_{k} \bar{\xi}_{l} \in \Sigma_{\theta}
$$

for all $\left(\xi_{1}, \ldots, \xi_{d}\right) \in \mathbb{C}^{d}$ and $x \in \mathbb{R}^{d}$, where

$$
\Sigma_{\theta}=\left\{r e^{i \alpha}: r \geq 0 \text { and } \alpha \in[-\theta, \theta]\right\} .
$$

Define the form $\mathfrak{a}: W^{1,2}\left(\mathbb{R}^{d}\right) \times W^{1,2}\left(\mathbb{R}^{d}\right) \rightarrow \mathbb{C}$ by

$$
\mathfrak{a}(u, v)=\sum_{k, l=1}^{d} \int_{\mathbb{R}^{d}} c_{k l}\left(\partial_{k} u\right) \overline{\partial_{l} v}
$$

Then $\mathfrak{a}$ is a densely defined sectorial form. In general $\mathfrak{a}$ is not closable, but nevertheless one can assign a semigroup generator $A$ with $\mathfrak{a}$ as follows. If $u, f \in L_{2}\left(\mathbb{R}^{d}\right)$ then $u \in D(A)$ and $A u=f$ if and only if there exist $u_{1}, u_{2}, \ldots \in W^{1,2}\left(\mathbb{R}^{d}\right)$ such that $\lim u_{n}=u$ in $L_{2}\left(\mathbb{R}^{d}\right), \sup \operatorname{Re} \mathfrak{a}\left(u_{n}\right)<\infty$ and $\lim \mathfrak{a}\left(u_{n}, v\right)=(f, v)$ for all $v \in W^{1,2}\left(\mathbb{R}^{d}\right)$. The operator $A$ is well defined and is $m$-sectorial by Theorem 1.1 in [3]. If $\mathfrak{a}$ is closable then $A$ is the operator associated with the closure $\overline{\mathfrak{a}}$ of the form $\mathfrak{a}$ in the sense of Kato [15]. We call $A$ the sectorial degenerate differential operator with coefficients $\left(c_{k l}\right)$. Formally, $A=-\sum_{k, l} \partial_{l} c_{k l} \partial_{k}$. We denote by $S=\left(S_{t}\right)_{t>0}$ the contraction semigroup generated by $-A$ on $L_{2}\left(\mathbb{R}^{d}\right)$. Then $S$ is holomorphic on the open sector $\Sigma_{\theta_{\mathfrak{a}}}^{\circ}$, where throughout this paper we define $\theta_{\mathfrak{a}}=\frac{\pi}{2}-\theta$. Let $\Omega \subset \mathbb{R}^{d}$ open. We suppose that the coefficients of $A$ are strongly elliptic on $\Omega$, that is, there exists a $\mu>0$ such that

$$
\operatorname{Re} \sum_{k, l=1}^{d} c_{k l}(x) \xi_{k} \overline{\xi_{l}} \geq \mu|\xi|^{2}
$$

for all $\xi \in \mathbb{C}^{d}$ and a.e. $x \in \Omega$.

The main results of this paper are the following. The first theorem is for complex Hölder continuous coefficients on $\Omega$, but with a multiplication operator on both sides of the semigroup. 
Theorem 1.1. Let $A$ be a sectorial degenerate differential operator with coefficients $\left(c_{k l}\right)$, where $c_{k l}: \mathbb{R}^{d} \rightarrow \mathbb{C}$ is a bounded measurable function for all $k, l \in$ $\{1, \ldots, d\}$. Let $\Omega \varsubsetneqq \mathbb{R}^{d}$ be open and suppose that $\left(c_{k l}\right)$ is strongly elliptic on $\Omega$. Let $v \in(0,1)$ and suppose that $c_{k l \mid \Omega} \in C^{0, v}(\Omega)$ for all $k, l \in\{1, \ldots, d\}$. Let $\chi \in C_{\mathrm{b}}^{\infty}\left(\mathbb{R}^{d}\right)$ with $\chi \neq 0$ and suppose $d\left(\operatorname{supp} \chi, \Omega^{\mathrm{c}}\right)>0$. Then there exists $a$ continuous function $(z, x, y) \mapsto K_{z}(x, y)$ from $\Sigma_{\theta_{\mathfrak{a}}}^{\circ} \times \mathbb{R}^{d} \times \mathbb{R}^{d}$ into $\mathbb{C}$ such that the following is valid.

- The function $K_{z}$ is the kernel of the operator $M_{\chi} S_{z} M_{\chi}$ for all $z \in \Sigma_{\theta_{a}}^{\circ}$, where $S$ is the semigroup generated by $-A$.

- The function $K_{z}$ is once differentiable in each variable and the derivative with respect to one variable is differentiable in the other variable.

- For every multi-index $\alpha, \beta$ with $|\alpha|,|\beta| \leq 1, \kappa>0$ and $\tau \in[0,1)$ there exist $a, b>0$ such that

$$
\left|\left(\partial_{x}^{\alpha} \partial_{y}^{\beta} K_{z}\right)(x, y)\right| \leq a|z|^{-d / 2}|z|^{-(|\alpha|+|\beta|) / 2}(1+|z|)^{\frac{d+|\alpha|+|\beta|}{2}} e^{-b \frac{|x-y|^{2}}{|z|}}
$$

and

$$
\begin{aligned}
& \left|\left(\partial_{x}^{\alpha} \partial_{y}^{\beta} K_{z}\right)(x+h, y+k)-\left(\partial_{x}^{\alpha} \partial_{y}^{\beta} K_{z}\right)(x, y)\right| \\
\leq & a|z|^{-d / 2}|z|^{-(|\alpha|+|\beta|) / 2}\left(\frac{|h|+|k|}{|x-y|+\sqrt{|z|}}\right)^{v}(1+|z|)^{\frac{d+|\alpha|+|\beta|+v}{2}} e^{-b \frac{|x-y|^{2}}{|z|}}
\end{aligned}
$$

for all $z \in \Sigma_{\theta_{\mathfrak{a}}}^{\circ}$ and $x, y, h, k \in \mathbb{R}^{d}$ with $|h|+|k| \leq \tau|x-y|+\kappa \sqrt{|z|}$.

The second result is for merely one multiplication operator on the left of the semigroup, but it requires that the coefficients of the operator are real on $\mathbb{R}^{d}$ and uniformly Lipschitz on $\Omega$.

Theorem 1.2. Let A be a sectorial degenerate differential operator with real coefficients $\left(c_{k l}\right)$, where $c_{k l}: \mathbb{R}^{d} \rightarrow \mathbb{R}$ is a bounded measurable function for all $k, l \in$ $\{1, \ldots, d\}$. Let $S$ be the semigroup generated by - A. Let $\Omega \varsubsetneqq \mathbb{R}^{d}$ be open and suppose that $\left(c_{k l}\right)$ is strongly elliptic on $\Omega$. Suppose that $c_{k l \mid \Omega} \in W^{1, \infty}(\Omega)$ for all $k, l \in$ $\{1, \ldots, d\}$. Let $\chi \in C_{\mathrm{b}}^{\infty}\left(\mathbb{R}^{d}\right)$ with $\chi \neq 0$ and suppose $d\left(\operatorname{supp} \chi, \Omega^{\mathrm{c}}\right)>0$. Then there exists a measurable function $(t, x, y) \mapsto K_{t}(x, y)$ from $(0, \infty) \times \mathbb{R}^{d} \times \mathbb{R}^{d}$ into $\mathbb{R}$ such that the following is valid.

- The function $K_{t}$ is a kernel of $M_{\chi} S_{t}$ for all $t>0$.

- The function $x \mapsto K_{t}(x, y)$ is continuously differentiable on $\mathbb{R}^{d}$ for all $t>0$ and $y \in \mathbb{R}^{d}$.

- The function $t \mapsto K_{t}(x, y)$ is continuous for all $x, y \in \mathbb{R}^{d}$.

- For every multi-index $\alpha$ with $|\alpha| \leq 1, v \in(0,1), \varepsilon>0, \kappa>0$ and $\tau \in[0,1)$ there exist $a, b>0$ such that

$$
\left|\left(\partial_{x}^{\alpha} K_{t}\right)(x, y)\right| \leq a t^{-d / 2} t^{-|\alpha| / 2} e^{\varepsilon t} e^{-b \frac{|x-y|^{2}}{t}}
$$


and

$$
\left|\left(\partial_{x}^{\alpha} K_{t}\right)(x+h, y)-\left(\partial_{x}^{\alpha} K_{t}\right)(x, y)\right| \leq a t^{-d / 2} t^{-|\alpha| / 2}\left(\frac{|h|}{|x-y|+\sqrt{t}}\right)^{v} e^{\varepsilon t} e^{-b \frac{|x-y|^{2}}{t}}
$$

for all $t>0$ and $x, y, h \in \mathbb{R}^{d}$ with $|h| \leq \tau|x-y|+\kappa \sqrt{t}$.

In Theorem 1.1 the function $(t, x, y) \mapsto K_{t}(x, y)$ is continuous, whilst it is not clear whether the function $(t, x, y) \mapsto K_{t}(x, y)$ is continuous in the setting of Theorem 1.2. Likely, there even does not exists a continuous function which is equal to this function almost everywhere on $(0, \infty) \times \mathbb{R}^{d} \times \mathbb{R}^{d}$. On the other hand, we prove measurability jointly in the three variables and do not work with an equivalent class of functions, for which the representative changes all the time. Since there are uncountable many $y \in \mathbb{R}^{d}$ this complicates the proof.

We also investigate boundedness on $L_{p}$ of Riesz transform type operators. We obtain the following result.

Theorem 1.3. Let $A$ be a sectorial degenerate differential operator with complex coefficients $\left(c_{k l}\right)$. Let $\Omega \varsubsetneqq \mathbb{R}^{d}$ be open and suppose that $\left(c_{k l}\right)$ is strongly elliptic on $\Omega$. Let $\chi \in C_{\mathrm{b}}^{\infty}\left(\mathbb{R}^{d}\right)$ with $\chi \neq 0$ and $d\left(\operatorname{supp} \chi, \Omega^{\mathrm{c}}\right)>0$. Then one has the following.

(a) Let $v \in(0,1)$ and suppose that $c_{k l \mid \Omega} \in C^{0, v}(\Omega)$ for all $k, l \in\{1, \ldots, d\}$. Then the Riesz transforms $\nabla M_{\chi}(I+A)^{-1 / 2} M_{\chi}$ are bounded on $L_{p}\left(\mathbb{R}^{d}\right)$ for all $p \in(1, \infty)$.

(b) Suppose that $c_{k l \mid \Omega} \in W^{1, \infty}(\Omega)$ and $c_{k l}$ is real valued for all $k, l \in\{1, \ldots, d\}$. Then the Riesz transforms $\nabla M_{\chi}(I+A)^{-1 / 2}$ are bounded on $L_{p}\left(\mathbb{R}^{d}\right)$ for all $p \in(1, \infty)$.

(c) Let $v \in(0,1)$ and suppose that $c_{k l \mid \Omega} \in C^{0, v}(\Omega)$ for all $k, l \in\{1, \ldots, d\}$. Then the Riesz transforms $\nabla M_{\chi}(I+A)^{-1 / 2}$ are bounded on $L_{2}\left(\mathbb{R}^{d}\right)$.

Using Morrey and Campanato spaces we prove Theorem 1.1 as in [5], if the operator is strongly elliptic on $\mathbb{R}^{d}$ and the coefficients are Hölder continuous on $\mathbb{R}^{d}$. We carefully control all the constants and show that they depend only on the ellipticity constant on $\Omega$ and on the Hölder continuity of the coefficients on $\Omega$. Then the theorem follows by approximation arguments. We prove a quantitive version of Theorem 1.1 in Section 2.

Since

$$
M_{\chi}^{2} S_{t}=M_{\chi} S_{t} M_{\chi}+M_{\chi}\left[M_{\chi}, S_{t}\right]
$$

and one can use Theorem 1.1 to handle the first term, it suffices to obtain good estimates on the commutator to derive the bounds of Theorem 1.2. This is done in Section 3.

Finally, in Section 4 we prove the boundedness of the Riesz transforms of Theorem 1.3 and the boundedness of several other Riesz transforms. For strongly elliptic operators in divergence form with complex bounded measurable coefficients the boundedness of the Riesz transforms on $L_{2}\left(\mathbb{R}^{d}\right)$ was the longstanding 
open Kato problem until it was solved by Auscher-Hofmann-Lacey-McIntoshTchamitchian [4]. For Hölder continuous coefficients the Kato problem was solved earlier by McIntosh [16] and a simplified proof was given in [7]. In the proof of Theorem 1.3(a) we adapt this simplified proof. In [6] Theorem 1.2 we proved Theorem 1.3(a) for merely measurable coefficients, but with the restriction that the coefficients are real symmetric and $p \in(1,2]$.

In the proofs we need various times to transfer semigroup estimates into Gaussian bounds, with control of large time behaviour, using the Davies perturbation method. Note that we deduce polynomial growth for large time for the kernel bounds in Theorem 1.1. The techniques are more or less folklore, however scattered over the literature. In the appendix we collect them together for the convenience of the reader. Finally, by decomposing $\chi$ into its real and imaginary part, for simplicity we may and do assume throughout the rest of this paper that the various cut-off functions $\chi, \tilde{\chi}, \ldots$ are always real valued.

\section{Complex Hölder continuous coefficients}

We start this section with the definition of a number of classes of coefficients and operators. The main aim is to obtain results for elements of these classes and that the constants involved are uniformly for a given class.

Let $\theta \in\left[0, \frac{\pi}{2}\right)$ and $M>0$. Define $\mathcal{S}(\theta, M)$ to be the set of all measurable $C: \mathbb{R}^{d} \rightarrow \mathbb{C}^{d \times d}$ such that

$$
\begin{aligned}
& \langle C(x) \xi, \xi\rangle \in \Sigma_{\theta} \text { for all } x \in \mathbb{R}^{d} \text { and } \xi \in \mathbb{C}^{d} \text {, and, } \\
& \|C(x)\| \leq M \quad \text { for all } x \in \mathbb{R}^{d},
\end{aligned}
$$

where $\|C(x)\|$ is the $\ell_{2}$-norm of $C(x)$ in $\mathbb{C}^{d}$ and $\langle\cdot, \cdot\rangle$ is the inner product on $\mathbb{C}^{d}$. For all $C \in \mathcal{S}(\theta, M)$ define the sectorial form form $\mathfrak{a}_{C}: W^{1,2}\left(\mathbb{R}^{d}\right) \times W^{1,2}\left(\mathbb{R}^{d}\right) \rightarrow \mathbb{C}$ by

$$
\mathfrak{a}_{C}(u, v)=\int_{\mathbb{R}^{d}} \sum_{k, l=1}^{d} c_{k l}\left(\partial_{k} u\right) \overline{\left(\partial_{l} v\right)}
$$

and let $A_{C}$ and $S^{C}$ be the associated operator and semigroup. Here and in the sequel $c_{k l}(x)$ is the appropriate matrix coefficient of $C(x)$. If no confusion is possible then we drop the $C$ and write $\mathfrak{a}=\mathfrak{a}_{C}, A=A_{C}$ and $S=S^{C}$. For all $C \in \mathcal{S}(\theta, M)$ define $\mathfrak{R C}: \mathbb{R}^{d} \rightarrow \mathbb{C}$ by

$$
(\mathfrak{R} C)(x)=\frac{1}{2}\left(C(x)+C(x)^{*}\right) .
$$

Then $\Re C \in \mathcal{S}(0, M)$ and $A_{\Re C}$ is self-adjoint. Moreover, $\mathfrak{a}_{\Re C}(u)=\operatorname{Re} \mathfrak{a}(u)$ for all $u \in W^{1,2}\left(\mathbb{R}^{d}\right)$. Next, let $\mathcal{E}(\theta, M)$ be the set of all $C \in \mathcal{S}(\theta, M)$ such that there exists a $\mu_{0}>0$ such that $\operatorname{Re}\langle C(x) \xi, \xi\rangle \geq \mu_{0}|\xi|^{2}$ for all $x \in \mathbb{R}^{d}$ and $\xi \in \mathbb{C}^{d}$. We emphasise that the constant $\mu_{0}$ depends on $C$. 
Let $Y \subset \mathbb{R}^{d}$ be a set, $\theta \in\left[0, \frac{\pi}{2}\right)$ and $\mu, M>0$. Let $\mathcal{S}(Y, \theta, \mu, M)$ be the set of all $C \in \mathcal{S}(\theta, M)$ such that

$$
\operatorname{Re}\langle C(x) \xi, \xi\rangle \geq \mu|\xi|^{2} \text { for all } x \in Y \text { and } \xi \in \mathbb{C}^{d}
$$

and define

$$
\mathcal{E}(Y, \theta, \mu, M)=\mathcal{S}(Y, \theta, \mu, M) \cap \mathcal{E}(\theta, M) .
$$

Next, let $v \in(0,1]$ and suppose that $Y$ contains at least two elements $x, y$ with $0<|x-y| \leq 1$. The space $C^{0, v}(Y)$ is the space of all Hölder continuous functions on $Y$ with seminorm

$$
\||| u||_{C^{0, \nu}(Y)}=\sup \left\{\frac{|u(x)-u(y)|}{|x-y|^{v}}: x, y \in Y, 0<|x-y| \leq 1\right\} .
$$

Let $\mathcal{S}^{v}(Y, \theta, \mu, M)$ be the set of all $C \in \mathcal{S}(Y, \theta, \mu, M)$ such that

$$
\left\|c_{k l \mid Y} \mid\right\|_{C^{0, v}(Y)} \leq M \text { for all } k, l \in\{1, \ldots, d\},
$$

and define

$$
\mathcal{E}^{v}(Y, \theta, \mu, M)=\mathcal{S}^{v}(Y, \theta, \mu, M) \cap \mathcal{E}(\theta, M) .
$$

Finally, let $\mathcal{E} \mathcal{H}^{v}(Y, \theta, \mu, M)$ be the set of all $C \in \mathcal{E}^{v}(Y, \theta, \mu, M)$ such that $\left\|\left|c_{k l}\right|\right\|_{C^{0, v}\left(\mathbb{R}^{d}\right)}<\infty$ for all $k, l \in\{1, \ldots, d\}$. If $C \in \mathcal{S}^{v}(Y, \theta, \mu, M)$ then $A_{C}$ is sectorial on $L_{2}\left(\mathbb{R}^{d}\right)$, whilst $A_{C}$ is strongly elliptic on $\mathbb{R}^{d}$ if $C \in \mathcal{E}^{v}(Y, \theta, \mu, M)$. Finally, $A_{C}$ is strongly elliptic with Hölder continuous coefficients on $\mathbb{R}^{d}$ if $C \in$ $\mathcal{E} \mathcal{H}^{v}(Y, \theta, \mu, M)$. In any case, $A_{C}$ is strongly elliptic on the set $Y$ with ellipticity constant at least $\mu$.

In the proof of the theorems we frequently need the Davies perturbation. For all $\rho \in \mathbb{R}$ and $\psi \in W^{1, \infty}\left(\mathbb{R}^{d}\right)$ define the multiplication operator $U_{\rho}$ by $U_{\rho} u=e^{-\rho \psi} u$. For all $n \in \mathbb{N}$ let

$$
\mathcal{D}_{n}=\left\{\psi \in W^{n, \infty}\left(\mathbb{R}^{d}, \mathbb{R}\right):\left\|\sum_{1 \leq|\alpha| \leq n}\left|\partial^{\alpha} \psi\right|^{2}\right\|_{\infty} \leq 1\right\} .
$$

Thus

$$
\mathcal{D}_{1}=\left\{\psi \in W^{1, \infty}\left(\mathbb{R}^{d}, \mathbb{R}\right):\|\nabla \psi\|_{\infty} \leq 1\right\} .
$$

Let $C \in \mathcal{S}(\theta, M)$. Define $S_{t}^{(C, \rho)}=S_{t}^{(\rho)}=U_{\rho} S_{t} U_{-\rho}$ to be the Davies perturbation of $S_{t}$ for all $t>0$. Let $-A_{C, \rho}=-A_{\rho}$ be the generator of $S^{(C, \rho)}$. Moreover, define the form $\mathfrak{a}_{C, \rho}$ by

$$
\mathfrak{a}_{C, \rho}(u, v)=\mathfrak{a}_{C}\left(U_{-\rho} u, U_{\rho} v\right)
$$

with form domain $D\left(\mathfrak{a}_{C, \rho}\right)=W^{1,2}\left(\mathbb{R}^{d}\right)$. Then $A_{C, \rho}$ is the operator associated with $\mathfrak{a}_{C, \rho}$

We frequently need the following lemma for estimates on $L_{2}$. 
Lemma 2.1. Let $\theta \in\left[0, \frac{\pi}{2}\right)$ and $\mu, M>0$. Then one has the following.

(a) If $C \in \mathcal{S}(\theta, M)$ then $\left\|S_{t}^{(\rho)}\right\|_{2 \rightarrow 2} \leq e^{\omega \rho^{2} t}$ for all $t>0, \psi \in \mathcal{D}_{1}$ and $\rho \in \mathbb{R}$, where $\omega=3(1+\tan \theta)^{2}\left(1+d^{2} M\right)$.

(b) Let $Y \subset \mathbb{R}^{d}$ and $\alpha \in\left(-\theta_{\mathfrak{a}}, \theta_{\mathfrak{a}}\right)$. Then

$$
e^{i \alpha} C \in \mathcal{S}\left(Y, \theta+|\alpha|, \mu \frac{\cos (\theta+|\alpha|)}{\cos \theta}, M\right)
$$

for all $C \in \mathcal{S}(Y, \theta, \mu, M)$.

(c) If $\chi \in W^{1, \infty}\left(\mathbb{R}^{d}\right)$ and $C \in \mathcal{S}(\operatorname{supp} \chi, \theta, \mu, M)$, then $M_{\chi} D\left(A_{\Re C}^{1 / 2}\right) \subset W^{1,2}\left(\mathbb{R}^{d}\right)$ and

$$
\sum_{m=1}^{d}\left\|\left(\partial_{m} M_{\chi}-M_{\partial_{m} \chi}\right) u\right\|_{2}^{2} \leq \frac{\|\chi\|_{\infty}^{2}}{\mu}\left\|A_{\Re C}^{1 / 2} u\right\|_{2}^{2}
$$

for all $u \in D\left(A_{\Re C}^{1 / 2}\right)$.

(d) If $\chi \in W^{1, \infty}\left(\mathbb{R}^{d}\right)$ and $C \in \mathcal{S}(\operatorname{supp} \chi, \theta, \mu, M)$, then $M_{\chi} S_{t} L_{2}\left(\mathbb{R}^{d}\right) \subset W^{1,2}\left(\mathbb{R}^{d}\right)$ and

$$
\left\|\left(\partial_{m} M_{\chi}-M_{\partial_{m} \chi}\right) S_{t} u\right\|_{2} \leq \frac{\|\chi\|_{\infty}}{\sqrt{\mu \sin \theta_{\mathfrak{a}}}} t^{-1 / 2}\|u\|_{2}
$$

for all $t>0, u \in L_{2}\left(\mathbb{R}^{d}\right)$ and $m \in\{1, \ldots, d\}$.

Remark 2.2. Note that $\partial_{m} M_{\chi}-M_{\partial_{m} \chi} \supset M_{\chi} \partial_{m}$ in Statements (c) and (d).

The rotational invariance of Statement (b) allows to consider various bounds on kernels $K_{z}$ merely for $z \in(0, \infty)$. Then the uniform bounds for $z$ in a sector $\Sigma_{\theta^{\prime}}^{\circ}$ with $\theta^{\prime} \in\left(0, \theta_{\mathfrak{a}}\right)$ follow since all bounds depend only on $\chi, \Omega, \mu, M, v$ and $\theta$.

Proof. (a). This follows from (14) in [3].

(b). Let $x \in Y$ and $\xi \in \mathbb{C}^{d}$. Then

$$
\begin{aligned}
\operatorname{Re}\left\langle e^{i \alpha} C(x) \xi, \xi\right\rangle & =\cos \alpha \operatorname{Re}\langle C(x) \xi, \xi\rangle-\sin \alpha \operatorname{Im}\langle C(x) \xi, \xi\rangle \\
& \geq \cos \alpha \operatorname{Re}\langle C(x) \xi, \xi\rangle-\sin |\alpha| \tan \theta \operatorname{Re}\langle C(x) \xi, \xi\rangle \\
& =\frac{\cos (\theta+|\alpha|)}{\cos \theta} \operatorname{Re}\langle C(x) \xi, \xi\rangle \\
& \geq \mu|\xi|^{2} \frac{\cos (\theta+|\alpha|)}{\cos \theta}
\end{aligned}
$$

This proves Statement (b).

(c). Let $\varepsilon>0$ and $n \in \mathbb{N}$. Set $C_{n}=C+\frac{1}{n} I$. Then $C_{n} \in \mathcal{E}(\operatorname{supp} \chi, \theta, \mu, M+1)$. 
Let $u \in W^{1,2}\left(\mathbb{R}^{d}\right)$. Then

$$
\begin{aligned}
\sum_{m=1}^{d}\left\|\left(\partial_{m} M_{\chi}-M_{\partial_{m} \chi}\right) u\right\|_{2}^{2} & =\sum_{m=1}^{d} \int\left|\chi \partial_{m} u\right|^{2} \\
& \leq \frac{1}{\mu} \int \operatorname{Re} \sum_{k, l=1}^{d}\left(c_{k l}+\frac{1}{n} \delta_{k l}\right) \chi^{2}\left(\partial_{k} u\right) \overline{\partial_{l} u} \\
& \leq \frac{\|\chi\|_{\infty}^{2}}{\mu}\left\|A_{\Re C_{n}}^{1 / 2} u\right\|_{2}^{2} \\
& \leq \frac{\|\chi\|_{\infty}^{2}}{\mu}\left\|\left(\varepsilon I+A_{\Re C_{n}}\right)^{1 / 2} u\right\|_{2}^{2} .
\end{aligned}
$$

Now let $u \in L_{2}\left(\mathbb{R}^{d}\right), v \in W^{1,2}\left(\mathbb{R}^{d}\right)$ and $m \in\{1, \ldots, d\}$. Then

$$
\begin{aligned}
\left|\left(\left(\varepsilon I+A_{\Re C_{n}}\right)^{-1 / 2} u, \partial_{m}(\chi v)\right)\right| & =\left|\left(\left(\partial_{m} M_{\chi}-M_{\partial_{m} \chi}\right)\left(\varepsilon I+A_{\Re C_{n}}\right)^{-1 / 2} u, v\right)\right| \\
& \leq \frac{\|\chi\|_{\infty}^{2}}{\mu}\|u\|_{2}\|v\|_{2} .
\end{aligned}
$$

Taking the limit $n \rightarrow \infty$ and using [3, Theorem 3.7] (cf. [19, Theorem 3.2]), one deduces that

$$
\left|\left(\left(\varepsilon I+A_{\Re C}\right)^{-1 / 2} u, \partial_{m}(\chi v)\right)\right| \leq \frac{\|\chi\|_{\infty}^{2}}{\mu}\|u\|_{2}\|v\|_{2} .
$$

So

$$
\left|\left(u, \partial_{m}(\chi v)\right)\right| \leq \frac{\|\chi\|_{\infty}^{2}}{\mu}\left\|\left(\varepsilon I+A_{\Re C}\right)^{1 / 2} u\right\|_{2}\|v\|_{2}
$$

for all $u \in D\left(A_{\mathfrak{R} C}^{1 / 2}\right), v \in W^{1,2}\left(\mathbb{R}^{d}\right)$ and $m \in\{1, \ldots, d\}$. Taking the limit $\varepsilon \downarrow 0$ one obtains

$$
\left|\left(u,\left(M_{\chi} \partial_{m}+M_{\partial_{m} \chi}\right) v\right)\right| \leq \frac{\|\chi\|_{\infty}^{2}}{\mu}\left\|A_{\Re C}^{1 / 2} u\right\|_{2}\|v\|_{2} .
$$

Therefore

$$
\left|\left(M_{\chi} u, \partial_{m} v\right)\right| \leq\left(\frac{\|\chi\|_{\infty}^{2}}{\mu}\left\|A_{\Re C}^{1 / 2} u\right\|_{2}+\left\|\partial_{m} \chi\right\|_{\infty}\|u\|_{2}\right)\|v\|_{2}
$$

for all $v \in W^{1,2}\left(\mathbb{R}^{d}\right)$ and $m \in\{1, \ldots, d\}$. So $M_{\chi} u \in W^{1,2}\left(\mathbb{R}^{d}\right)$ and then the estimate (2.1) follows from (2.2).

(d). Let $\alpha \in\left(0, \theta_{\mathfrak{a}}\right)$. Then it follows from Statements (a) and (b) that $\left\|S_{z}^{(\rho)}\right\|_{2 \rightarrow 2} \leq$ $e^{\omega \rho^{2}|z|}$ for all $z \in \Sigma_{\alpha}^{\circ}, \rho \in \mathbb{R}$ and $\psi \in \mathcal{D}_{1}$, where $\omega=3(1+\tan (\theta+\alpha))^{2}\left(1+d^{2} M\right)$. Hence the Cauchy representation formula gives

$$
\left\|A_{\rho} S_{t}^{(\rho)}\right\|_{2 \rightarrow 2} \leq \frac{1}{t \sin \alpha} e^{2 \omega \rho^{2} t}
$$


for all $t>0, \rho \in \mathbb{R}$ and $\psi \in \mathcal{D}_{1}$. If $C \in \mathcal{E}(\operatorname{supp} \chi, \theta, \mu, M)$ then

$$
\begin{aligned}
\left\|\left(\partial_{m} M_{\chi}-M_{\partial_{m} \chi}\right) S_{t}^{C} u\right\|_{2}^{2} & \leq \frac{\|\chi\|_{\infty}^{2}}{\mu}\left\|A_{\Re C}^{1 / 2} S_{t}^{C} u\right\|_{2}^{2} \\
& =\frac{\|\chi\|_{\infty}^{2}}{\mu} \operatorname{Re}\left(A_{C} S_{t}^{C} u, S_{t}^{C} u\right) \\
& \leq \frac{\|\chi\|_{\infty}^{2}}{\mu}\left\|A_{C} S_{t}^{C} u\right\|_{2}\left\|S_{t}^{C} u\right\|_{2} \\
& \leq \frac{\|\chi\|_{\infty}^{2}}{\mu} \frac{1}{t \sin \alpha}\|u\|_{2}^{2} .
\end{aligned}
$$

Now take the limit $\alpha \uparrow \theta_{\mathfrak{a}}$. Finally, let $C \in \mathcal{S}(\operatorname{supp} \chi, \theta, \mu, M)$. For all $n \in \mathbb{N}$ define $C_{n}=C+\frac{1}{n} I$. Since $\lim _{n \rightarrow \infty} S_{t}^{C_{n}}=S_{t}^{C}$ strongly in $\mathcal{L}\left(L_{2}\left(\mathbb{R}^{d}\right)\right)$ by [3] Corollary 3.9, now Statement (d) follows as in the proof of Statement (c).

The next theorem is a uniform version of Theorem 1.1.

Theorem 2.3. Let $\Omega \varsubsetneqq \mathbb{R}^{d}$ be open, $\theta \in\left[0, \frac{\pi}{2}\right), \mu, M>0, v \in(0,1), \kappa>0$, $\tau \in[0,1), \theta^{\prime} \in\left(0, \theta_{\mathfrak{a}}\right)$ and $\chi \in C_{\mathrm{b}}^{\infty}\left(\mathbb{R}^{d}\right)$ with $\chi \neq 0$ and $d\left(\operatorname{supp} \chi, \Omega^{\mathrm{c}}\right)>0$. Then there exist $a, b>0$ such that for every $C \in \mathcal{S}^{\nu}(\Omega, \theta, \mu, M)$ there exists a function $(z, x, y) \mapsto K_{z}(x, y)$ from $\Sigma_{\theta_{\mathfrak{a}}}^{\circ} \times \mathbb{R}^{d} \times \mathbb{R}^{d}$ into $\mathbb{C}$ such that the following is valid.

(a) The function $(z, x, y) \mapsto K_{z}(x, y)$ is continuous from $\Sigma_{\theta_{\mathfrak{a}}}^{\circ} \times \mathbb{R}^{d} \times \mathbb{R}^{d}$ into $\mathbb{C}$.

(b) For all $z \in \Sigma_{\theta^{\prime}}^{\circ}$ the function $K_{z}$ is the kernel of the operator $M_{\chi} S_{z} M_{\chi}$.

(c) For all $x, y \in \mathbb{R}^{d}$ the function $z \mapsto K_{z}(x, y)$ is holomorphic from $\Sigma_{\theta^{\prime}}^{\circ}$ into $\mathbb{C}$.

(d) For all $z \in \Sigma_{\theta^{\prime}}^{\circ}$ the function $K_{z}$ is once differentiable in each variable and the derivative with respect to one variable is differentiable in the other variable. Moreover, for every multi-index $\alpha, \beta$ with $0 \leq|\alpha|,|\beta| \leq 1$ one has

$$
\left|\left(\partial_{x}^{\alpha} \partial_{y}^{\beta} K_{z}\right)(x, y)\right| \leq a|z|^{-d / 2}|z|^{-(|\alpha|+|\beta|) / 2}(1+|z|)^{\frac{d+|\alpha|+|\beta|}{2}} e^{-b \frac{|x-y|^{2}}{|z|}}
$$

and

$$
\begin{aligned}
& \left|\left(\partial_{x}^{\alpha} \partial_{y}^{\beta} K_{z}\right)(x+h, y+k)-\left(\partial_{x}^{\alpha} \partial_{y}^{\beta} K_{z}\right)(x, y)\right| \\
\leq & a|z|^{-d / 2}|z|^{-(|\alpha|+|\beta|) / 2}\left(\frac{|h|+|k|}{|x-y|+\sqrt{|z|}}\right)^{v}(1+|z|)^{\frac{d+|\alpha|+|\beta|+v}{2}} e^{-b \frac{|x-y|^{2}}{|z|}}
\end{aligned}
$$

for all $x, y, h, k \in \mathbb{R}^{d}$ with $|h|+|k| \leq \tau|x-y|+\kappa \sqrt{|z|}$.

Proof. We first prove the theorem with $\mathcal{S}^{\nu}(\Omega, \theta, \mu, M)$ replaced by $\mathcal{E} \mathcal{H}^{\nu}(\Omega, \theta, \mu, M)$. For strongly elliptic operators on $\mathbb{R}^{d}$ in divergence form and Hölder continuous coefficients all the kernels with stated holomorphy and continuity properties are well known. The main point is to derive the uniform bounds. We emphasise that the 
constants in the proof do not depend on the ellipticity constant $\mu_{0}$ for elements in $\mathcal{E} \mathcal{H}^{v}(\Omega, \theta, \mu, M)$, nor on the Hölder continuity of the coefficients on $\Omega^{\mathrm{c}}$. Then we will approximate elements of $\mathcal{E}^{\nu}(\Omega, \theta, \mu, M)$ by elements of $\mathcal{E} \mathcal{H}^{\nu}(\Omega, \theta, \mu, M)$ and finally approximate elements of $\mathcal{S}^{v}(\Omega, \theta, \mu, M)$ by elements of $\mathcal{E}^{v}(\Omega, \theta, \mu, M)$.

Without loss of generality we may assume that $d \geq 3$. Let $F \subset \Omega$ be a closed set with $F \neq \varnothing$ and $d\left(F, \Omega^{\mathrm{c}}\right)>0$. Let $r_{0}=\frac{1}{3} d\left(F, \Omega^{\mathrm{c}}\right)$. There exist $\chi_{1}, \chi_{2} \in C_{\mathrm{b}}^{\infty}\left(\mathbb{R}^{d}\right)$ such that $0 \leq \chi_{1}, \chi_{2} \leq 11$ and

$$
\begin{aligned}
& \chi_{1}(x)=\left\{\begin{array}{l}
0 \text { if } d(x, F)<r_{0}, \\
1 \text { if } d\left(x, \Omega^{\mathrm{c}}\right)<r_{0},
\end{array}\right. \\
& \chi_{2}(x)=\left\{\begin{array}{l}
1 \text { if } d(x, F)<2 r_{0}, \\
0 \text { if } x \notin \Omega,
\end{array}\right.
\end{aligned}
$$

for all $x \in \mathbb{R}^{d}$. Let $M^{\prime}=2\left\|\chi_{2}\right\|_{W^{1, \infty}\left(\mathbb{R}^{d}\right)} M+\left\|\chi_{1}\right\|_{W^{1, \infty}\left(\mathbb{R}^{d}\right)}+1$. Let $C \in$ $\mathcal{E} \mathcal{H}^{v}(\Omega, \theta, \mu, M)$. Define $C^{\prime}=\chi_{2} C+\chi_{1} I$. Then $C^{\prime} \in \mathcal{E} \mathcal{H}^{v}\left(\mathbb{R}^{d}, \theta, \mu \wedge 1, M^{\prime}\right)$. Since the operator $A_{C^{\prime}}$ is a strongly elliptic operator on $\mathbb{R}^{d}$ with $C^{0, v}$-coefficients, it satisfies various kinds of De Giorgi estimates. On bounded open sets these are proved by Giaquinta [11,12], or Xu-Zuily [21]. The global estimates follow from [9, Proposition 3.5] and [8, Proposition 2.6]. Precisely, there exist $c_{D G}^{\prime}, c_{D G}^{\prime \prime}>0$ and for all $v^{\prime} \in(0,1)$ a $c_{D G}>0$, depending only on $\mu, M^{\prime}, v$ and $v^{\prime}$, such that

$$
\int_{B(x, r)}|\nabla u|^{2} \leq c_{D G}\left(\frac{r}{R}\right)^{d-2+2 v^{\prime}} \int_{B(x, R)}|\nabla u|^{2}
$$

and

$$
\begin{aligned}
& \sum_{k=1}^{d} \int_{B(x, r)}\left|\partial_{k} u-\left\langle\partial_{k} u\right\rangle_{x, r}\right|^{2} \\
& \leq c_{D G}^{\prime}\left(\frac{r}{R}\right)^{d+2 \tilde{v}} \sum_{k=1}^{d} \int_{B(x, R)}\left|\partial_{k} u-\left\langle\partial_{k} u\right\rangle_{x, R}\right|^{2}+c_{D G}^{\prime \prime} R^{2 v} \sum_{k=1}^{d} \int_{B(x, R)}|\nabla u|^{2}
\end{aligned}
$$

for all $R \in(0,1], r \in(0, R]$ and $u \in W^{1,2}(B(x, R))$ satisfying $A_{C^{\prime}} u=0$ weakly on $B(x, R)$, where $\tilde{v}=\frac{1}{2}(1+v)$.

Write $\mathfrak{a}=\mathfrak{a}_{C}, A=A_{C}$ and $S=S^{C}$. It is well known that the semigroup generated by $S$ has a kernel $K^{S}$ satisfying continuity, holomorphy and Gaussian properties similar to (a)-(d) in the theorem. Define $K_{z}(x, y)=\chi(x) K_{z}^{S}(x, y) \chi(y)$. Then $K_{z}$ is the kernel of $M_{\chi} S_{z} M_{\chi}$. Moreover, $K$ satisfies Properties (a)-(c).

Note that $\mathfrak{a}(u, v)=\mathfrak{a}_{C^{\prime}}(u, v)$ for all $u, v \in W^{1,2}\left(\mathbb{R}^{d}\right)$ with supp $u \subset F$. For all $\gamma \in[0, d]$ let $M_{2, \gamma}\left(\mathbb{R}^{d}\right)$ be the Morrey space and for all $\gamma \in[0, d+2)$ let $\mathcal{M}_{2, \gamma}\left(\mathbb{R}^{d}\right)$ be the Campanato space as defined in [5] Section 2. 
For all $\gamma \in[0, d)$ let $P(\gamma)$ be the following hypothesis:

For all $\chi \in C_{\mathrm{b}}^{\infty}\left(\mathbb{R}^{d}\right)$ with supp $\chi \subset F$ there exist $a_{1}, \omega_{1}>0$, depending only on $\chi, \Omega, \theta, \mu, M$ and $v$, such that

$$
\left\|M_{\chi} S_{t}^{(\rho)} u\right\|_{M_{2, \gamma}\left(\mathbb{R}^{d}\right)} \leq a_{1} t^{-\gamma / 4} e^{\omega_{1}\left(1+\rho^{2}\right) t}\|u\|_{2}
$$

and

$$
\left\|\nabla M_{\chi} S_{t}^{(\rho)} u\right\|_{M_{2, \gamma}\left(\mathbb{R}^{d}\right)} \leq a_{1} t^{-\gamma / 4} t^{-1 / 2} e^{\omega_{1}\left(1+\rho^{2}\right) t}\|u\|_{2}
$$

uniformly for all $t>0, u \in L_{2}\left(\mathbb{R}^{d}\right), \rho \in \mathbb{R}$ and $\psi \in \mathcal{D}_{1}$.

Arguing as in the proof of Lemma 3.3 in [5] it follows from (2.3) and the De Giorgi estimates (2.6) that $P(\gamma)$ is valid for all $\gamma \in[0, d)$.

Next, for all $\gamma \in[0, d+2 v]$ let $P^{\prime}(\gamma)$ be the following hypothesis:

For all $\chi \in C_{\mathrm{b}}^{\infty}\left(\mathbb{R}^{d}\right)$ with supp $\chi \subset F$ there exist $a_{1}, \omega_{1}>0$, depending only on $\chi, \Omega, \theta, \mu, M$ and $v$, such that

$$
\left\|M_{\chi} S_{t}^{(\rho)} u\right\|_{\mathcal{M}_{2, \gamma}\left(\mathbb{R}^{d}\right)} \leq a_{1} t^{-\gamma / 4} e^{\omega_{1}\left(1+\rho^{2}\right) t}\|u\|_{2}
$$

and

$$
\left\|\nabla M_{\chi} S_{t}^{(\rho)} u\right\|_{\mathcal{M}_{2, \gamma}\left(\mathbb{R}^{d}\right)} \leq a_{1} t^{-\gamma / 4} t^{-1 / 2} e^{\omega_{1}\left(1+\rho^{2}\right) t}\|u\|_{2}
$$

uniformly for all $t>0, u \in L_{2}\left(\mathbb{R}^{d}\right), \rho \in \mathbb{R}$ and $\psi \in \mathcal{D}_{2}$.

Since $M_{2, \gamma} \cap L_{2}=\mathcal{M}_{2, \gamma} \cap L_{2}$ for all $\gamma \in[0, d)$, with equivalent norms, one deduces from $P(\gamma)$ that also $P^{\prime}(\gamma)$ is valid for all $\gamma \in[0, d)$. But arguing as in [8], proof of Proposition 3.2 and the proof of Lemma 3.3 in [5] it follows from the De Giorgi estimates (2.7) that $P^{\prime}(\gamma)$ is valid for all $\gamma \in[0, d+2 v]$. Hence there are $a, \omega>0$, depending only on $\chi, \Omega, \theta, \mu, M$ and $\nu$, such that

$$
\left\|\partial^{\alpha} M_{\chi} S_{t}^{(\rho)} u\right\|_{\infty} \leq a t^{-d / 4} t^{-|\alpha| / 2} e^{\omega\left(1+\rho^{2}\right) t}\|u\|_{2}
$$

and

$$
\left\|(I-L(h)) \partial^{\alpha} M_{\chi} S_{t}^{(\rho)} u\right\|_{\infty} \leq a t^{-d / 4} t^{-|\alpha| / 2}\left(\frac{|h|}{\sqrt{t}}\right)^{\nu} e^{\omega\left(1+\rho^{2}\right) t}\|u\|_{2}
$$

for all multi-indices $\alpha$ with $|\alpha| \leq 1, t>0, u \in L_{2}\left(\mathbb{R}^{d}\right), \rho \in \mathbb{R}$ and $\psi \in \mathcal{D}_{2}$. Here $L(h)$ denotes left translation, defined by $(L(h) u)(x)=u(x-h)$. Next, $\left\|S_{t}^{(\rho)}\right\|_{2 \rightarrow 2} \leq e^{\omega_{0} \rho^{2} t}$ for all $t>0$, where $\omega_{0}=3(1+\tan \theta)^{2}\left(1+d^{2} M\right)$ by Lemma 2.1(a).

Then the bounds of Property (d) follow from Lemma A.1 in Appendix A uniformly for all $z \in \Sigma_{\theta^{\prime}}^{\circ}$ and $C \in \mathcal{E} \mathcal{H}^{\nu}(\Omega, \theta, \mu, M)$. This proves the theorem with $\mathcal{S}^{\nu}(\Omega, \theta, \mu, M)$ replaced by $\mathcal{E H}^{\nu}(\Omega, \theta, \mu, M)$. 
Let $\chi \in C_{\mathrm{b}}^{\infty}\left(\mathbb{R}^{d}\right)$ with $\chi \neq 0$ and $d\left(\operatorname{supp} \chi, \Omega^{\mathrm{c}}\right)>0$. Let $r_{0}=\frac{1}{2} d\left(\operatorname{supp} \chi, \Omega^{\mathrm{c}}\right)$ and let $\Omega^{\prime}=\left\{x \in \Omega: d(x, \operatorname{supp} \chi)<r_{0}\right\}$. Then $\Omega^{\prime}$ is open, $d\left(\operatorname{supp} \chi,\left(\Omega^{\prime}\right)^{\mathfrak{c}}\right)>0$ and $d\left(\Omega^{\prime}, \Omega^{\mathrm{c}}\right) \geq r_{0}>0$.

Let $\alpha, \beta$ be multi-indices with $|\alpha|,|\beta| \leq 1$. Using the Cauchy representation formula on the sector $\Sigma_{\theta_{\mathfrak{a}}}^{\circ}$ one deduces that $\partial_{t} \partial_{x}^{\alpha} \partial_{y}^{\beta} K_{z}^{C}$ satisfies Hölder type Gaussian bounds uniformly for all $C \in \mathcal{E} \mathcal{H}^{v}\left(\Omega^{\prime}, \theta, \mu, M\right)$, where $K^{C}$ is the kernel associated with $z \mapsto M_{\chi} S_{z}^{C} M_{\chi}$. Hence the set of functions

$$
\left\{(z, x, y) \mapsto\left(\partial_{x}^{\alpha} \partial_{y}^{\beta} K_{z}^{C}\right)(x, y): C \in \mathcal{E} \mathcal{H}^{\nu}\left(\Omega^{\prime}, \theta, \mu, M\right)\right\}
$$

is equicontinuous on compact subsets of $\Sigma_{\theta_{\mathfrak{a}}}^{\circ} \times \mathbb{R}^{d} \times \mathbb{R}^{d}$.

Fix $\tau \in C_{c}^{\infty}\left(B\left(0, r_{0}\right)\right)$ with $\tau \geq 0$ and $\int \tau=1$. For all $n \in \mathbb{N}$ define $\tau_{n} \in C_{c}^{\infty}\left(\mathbb{R}^{d}\right)$ by $\tau_{n}(x)=n^{d} \tau(n x)$. Now let $C \in \mathcal{E}^{\nu}(\Omega, \theta, \mu, M)$. For all $n \in \mathbb{N}$ and $k, l \in\{1, \ldots, d\}$ define $c_{k l}^{(n)}=c_{k l} * \tau_{n}$ and define $C^{(n)}=\left(c_{k l}^{(n)}\right)$. Then $C^{(n)} \in \mathcal{E} \mathcal{H}^{\nu}\left(\Omega^{\prime}, \theta, \mu, M\right)$ for all $n \in \mathbb{N}$. Write $S^{(n)}=S^{C^{(n)}}$, etc. Since $\left\{(z, x, y) \mapsto\left(\partial_{x}^{\alpha} \partial_{y}^{\beta} K_{z}^{(n)}\right)(x, y): n \in \mathbb{N}\right\}$ is equicontinuous on compact subsets of $\Sigma_{\theta_{\mathfrak{a}}}^{\circ} \times \mathbb{R}^{d} \times \mathbb{R}^{d}$ for all $|\alpha|,|\beta| \leq 1$ it follows with a diagonal argument from the Arzéla-Ascoli theorem that there exists a subsequence $\left(K^{\left(n_{k}\right)}\right)_{k \in \mathbb{N}}$ of $\left(K^{(n)}\right)_{n \in \mathbb{N}}$ such that $K^{(\alpha, \beta)}=\lim _{k \rightarrow \infty} \partial_{x}^{\alpha} \partial_{y}^{\beta} K^{\left(n_{k}\right)}$ exists uniformly on compact subsets of $\Sigma_{\theta_{\mathfrak{a}}}^{\circ} \times \mathbb{R}^{d} \times \mathbb{R}^{d}$ and every multi-index $\alpha, \beta$ with $|\alpha|,|\beta| \leq 1$. Then $(z, x, y) \mapsto K_{z}^{(\alpha, \beta)}(x, y)$ is continuous on $\Sigma_{\theta_{\mathfrak{a}}}^{\circ} \times \mathbb{R}^{d} \times \mathbb{R}^{d}$. Set $K=K^{(\alpha, \beta)}$ where $|\alpha|=|\beta|=0$. Obviously for every $z \in \Sigma_{\theta_{\mathfrak{a}}}^{\circ}$ the function $K_{z}$ is once differentiable in each variable and the derivative with respect to one variable is differentiable in the other variable. Also $K_{z}$ satisfies all the Gaussian bounds from the theorem and the constants in the Gaussians depend only on $\chi, \Omega, \theta^{\prime}, \mu, M$ and $\nu$. Let $z \in \Sigma_{\theta_{\mathfrak{a}}}^{\circ}$. Let $u, v \in C_{c}^{\infty}\left(\mathbb{R}^{d}\right)$. We shall prove below in Lemma 2.4 that $\lim _{n \rightarrow \infty} S_{z}^{(n)} u=S_{z}^{C} u$ in $L_{2}\left(\mathbb{R}^{d}\right)$. Hence

$$
\begin{aligned}
\left(S_{z}^{C} u, v\right) & =\lim _{k \rightarrow \infty}\left(S_{z}^{\left(n_{k}\right)} u, v\right) \\
& =\lim _{k \rightarrow \infty} \iint K_{z}^{\left(n_{k}\right)}(x, y) u(y) \overline{v(x)} d x d y \\
& =\iint K_{z}(x, y) u(y) \overline{v(x)} d x d y .
\end{aligned}
$$

Since $K$ satisfies Gaussian bounds it follows that $K_{z}$ is the kernel of $S_{z}^{C}$. This proves the theorem with $\mathcal{S}^{\nu}(\Omega, \theta, \mu, M)$ replaced by $\mathcal{E}^{\nu}(\Omega, \theta, \mu, M)$.

Finally, let $C \in \mathcal{S}^{\nu}(\Omega, \theta, \mu, M)$. For all $n \in \mathbb{N}$ define $C_{n}=C+\frac{1}{n} I$. Then $C_{n} \in \mathcal{E}^{\nu}(\Omega, \theta, \mu, M+1)$ for all $n \in \mathbb{N}$. Since $\lim _{n \rightarrow \infty} S_{z}^{C_{n}} u=S_{z}^{C} u$ in $L_{2}\left(\mathbb{R}^{d}\right)$ for all $z \in \Sigma_{\theta_{\mathfrak{a}}}^{\circ}$ and $u \in L_{2}\left(\mathbb{R}^{d}\right)$ by [3] Corollary 3.9, a similar approximation argument as in the previous step completes the proof of Theorem 2.3.

It remains to show the next lemma. 
Lemma 2.4. Let $\theta \in\left[0, \frac{\pi}{2}\right)$ and $M>0$. Let $C \in \mathcal{E}(\theta, M)$ and $\tau \in C_{c}^{\infty}\left(\mathbb{R}^{d}\right)$ with $\tau \geq 0$ and $\int \tau=1$. For all $n \in \mathbb{N}$ define $\tau_{n} \in C_{c}^{\infty}\left(\mathbb{R}^{d}\right)$ by $\tau_{n}(x)=n^{d} \tau(n x)$ and set $C^{(n)}=\left(c_{k l}^{(n)}\right)$, where $c_{k l}^{(n)}=\tau_{n} * c_{k l}$ for all $k, l \in\{1, \ldots, d\}$. Then $C^{(n)} \in$ $\mathcal{E}(\theta, M)$ for all $n \in \mathbb{N}$. Write $S^{(n)}=S^{C^{(n)}}, S=S^{C}$, etc. Then $\lim _{n \rightarrow \infty} S_{z}^{(n)}=S_{z}$ strongly in $\mathcal{L}\left(L_{2}\left(\mathbb{R}^{d}\right)\right)$ for all $z \in \Sigma_{\theta_{\mathfrak{a}}}^{\circ}$.

Proof. Let $\eta \in L_{\infty}\left(\mathbb{R}^{d}\right)$ and $u \in L_{2}\left(\mathbb{R}^{d}\right)$. Then

$$
((I-L(x)) \eta) u=(I-L(x))(\eta u)-(L(x) \eta)(I-L(x)) u
$$

for all $x \in \mathbb{R}^{d}$. So

$$
\left\|\left(\eta-\tau_{n} * \eta\right) u\right\|_{2} \leq\left\|\eta u-\tau_{n} *(\eta u)\right\|_{2}+\|\eta\|_{\infty} \int_{\mathbb{R}^{d}} \tau_{n}(x)\|(I-L(x)) u\|_{2} d x
$$

for all $n \in \mathbb{N}$ and $\lim \left\|\left(\eta-\tau_{n} * \eta\right) u\right\|_{2}=0$.

There exists a $\mu_{0}>0$ such that $\operatorname{Re}\langle C(x) \xi, \xi\rangle \geq \mu_{0}|\xi|^{2}$ for all $x \in \mathbb{R}^{d}$ and $\xi \in \mathbb{C}^{d}$. Then $\operatorname{Re}\left\langle C^{(n)}(x) \xi, \xi\right\rangle \geq \mu_{0}|\xi|^{2}$ and $\left\|C^{(\bar{n})}(x)\right\| \leq M$ for all $x \in \mathbb{R}^{d}$, $\xi \in \mathbb{C}^{d}$ and $n \in \mathbb{N}$. Hence there exists a $c>0$ such that $\left\|\nabla S_{t}^{(n)} u\right\|_{2} \leq c t^{-1 / 2}\|u\|_{2}$ for all $t>0, u \in L_{2}\left(\mathbb{R}^{d}\right)$ and $n \in \mathbb{N}$ (cf. the proof of (13) in [5]). By increasing $c$ if necessary, it follows similarly that $\left\|\nabla S_{t} u\right\|_{2} \leq c t^{-1 / 2}\|u\|_{2}$, and $\left\|\nabla S_{t}^{(n) *} u\right\|_{2} \leq$ $c t^{-1 / 2}\|u\|_{2}$ for all $t>0$ and $u \in L_{2}\left(\mathbb{R}^{d}\right)$.

Without loss of generality we may assume that $z \in(0, \infty)$. Next, let $u, v \in$ $L_{2}\left(\mathbb{R}^{d}\right)$. Then

$$
\begin{aligned}
\left(\left(S_{z}^{(n)}-S_{z}\right) u, v\right) & =\int_{0}^{z} \frac{d}{d s}\left(S_{z-s} u, S_{s}^{(n) *} v\right) d s \\
& =\int_{0}^{z}\left(A S_{z-s} u, S_{s}^{(n) *} v\right)-\left(S_{z-s} u, A_{n}^{*} S_{s}^{(n) *} v\right) d s \\
& =\int_{0}^{z} \mathfrak{a}\left(\left(S_{z-s} u, S_{s}^{(n) *} v\right)-\mathfrak{a}_{n}\left(\left(S_{z-s} u, S_{s}^{(n) *} v\right) d s\right.\right. \\
& =\sum_{k, l=1}^{d} \int_{0}^{z}\left(\left(c_{k l}-c_{k l}^{(n)}\right) \partial_{k} S_{z-s} u, \partial_{l} S_{s}^{(n) *} v\right) d s
\end{aligned}
$$

for all $n \in \mathbb{N}$. So

$$
\left\|\left(S_{z}^{(n)}-S_{z}\right) u\right\|_{2} \leq c \sum_{k, l=1}^{d} \int_{0}^{z}\left\|\left(c_{k l}-c_{k l}^{(n)}\right) \partial_{k} S_{z-s} u\right\|_{2} s^{-1 / 2} d s
$$

and the lemma follows from the Lebesgue dominated convergence theorem.

The proof of Theorem 2.3 together with Lemma 2.4 gives estimates which we need in the proof of Proposition 3.7. 
Lemma 2.5. Let $\Omega \varsubsetneqq \mathbb{R}^{d}$ be open, $\theta \in\left[0, \frac{\pi}{2}\right), \mu, M>0, v \in(0,1)$ and $\chi \in$ $C_{\mathrm{b}}^{\infty}\left(\mathbb{R}^{d}\right)$ with $\chi \neq 0$ and $d\left(\operatorname{supp} \chi, \Omega^{\mathrm{c}}\right)>0$. Then there exist $a, \omega>0$ such that for every $C \in \mathcal{S}^{v}(\Omega, \theta, \mu, M)$ one has $M_{\chi} S_{t} u \in W^{1, \infty}\left(\mathbb{R}^{d}\right)$ and

$$
\left\|\partial^{\alpha} M_{\chi} S_{t} u\right\|_{\infty} \leq a t^{-d / 4} t^{-|\alpha| / 2} e^{\omega t}\|u\|_{2}
$$

for all multi-indices $\alpha$ with $|\alpha| \leq 1, t>0$ and $u \in L_{2}\left(\mathbb{R}^{d}\right)$.

Proof. Let $\chi \in C_{\mathrm{b}}^{\infty}\left(\mathbb{R}^{d}\right)$ with $\chi \neq 0$ and $d\left(\operatorname{supp} \chi, \Omega^{\mathrm{c}}\right)>0$. Let $r_{0}=\frac{1}{2} d\left(\operatorname{supp} \chi, \Omega^{\mathrm{c}}\right)$ and $\Omega^{\prime}=\left\{x \in \Omega: d(x, \operatorname{supp} \chi)<r_{0}\right\}$. By (2.8) there exist $a, \omega>0$ such that

$$
\left\|\partial^{\alpha} M_{\chi} S_{t}^{C} u\right\|_{\infty} \leq a t^{-d / 4} t^{-|\alpha| / 2} e^{\omega t}\|u\|_{2}
$$

for all $C \in \mathcal{E} \mathcal{H}^{\nu}(\Omega, \theta, \mu, M)$, multi-indices $\alpha$ with $|\alpha| \leq 1, t>0$ and $u \in L_{2}\left(\mathbb{R}^{d}\right)$. Let $C \in \mathcal{E}^{v}(\Omega, \theta, \mu, M)$. Let $\tau \in C_{c}^{\infty}\left(B\left(0, r_{0}\right)\right)$ with $\tau \geq 0$ and $\int \tau=1$. For all $n \in \mathbb{N}$ let $C^{(n)}$ be as in Lemma 2.4. Then $C^{(n)} \in \mathcal{E} \mathcal{H}^{\nu}(\Omega, \theta, \mu, M)$. Let $t>0$ and $u \in L_{2}\left(\mathbb{R}^{d}\right)$. Then

$$
\left|\left(S_{t}^{C^{(n)}} u, M_{\chi} \partial^{\alpha} v\right)\right|=\left|\left(\partial^{\alpha} M_{\chi} S_{t}^{C^{(n)}} u, v\right)\right| \leq a t^{-d / 4} t^{-|\alpha| / 2} e^{\omega t}\|u\|_{2}\|v\|_{1}
$$

for all multi-indices $\alpha$ with $|\alpha| \leq 1, v \in C_{c}^{\infty}\left(\mathbb{R}^{d}\right)$ and $n \in \mathbb{N}$. Now take the limit $n \rightarrow \infty$ and use Lemma 2.4. It follows that

$$
\left|\left(M_{\chi} S_{t}^{C} u, \partial^{\alpha} v\right)\right| \leq a t^{-d / 4} t^{-|\alpha| / 2} e^{\omega t}\|u\|_{2}\|v\|_{1}
$$

for all $v \in C_{c}^{\infty}\left(\mathbb{R}^{d}\right)$ and $|\alpha| \leq 1$. Choosing $|\alpha|=0$, it follows that $M_{\chi} S_{t} u \in$ $L_{\infty}\left(\mathbb{R}^{d}\right)$. Next $|\alpha|=1$ and the density of $C_{c}^{\infty}\left(\mathbb{R}^{d}\right)$ in $W^{1,1}\left(\mathbb{R}^{d}\right)$ give that $(2.10)$ is valid for all $v \in W^{1,1}\left(\mathbb{R}^{d}\right)$ and $|\alpha|=1$. Hence $M_{\chi} S_{t} u \in W^{1, \infty}\left(\mathbb{R}^{d}\right)$ and (2.9) is valid.

Finally, if $C \in \mathcal{S}^{v}(\Omega, \theta, \mu, M)$ use the approximation $C_{n}=C+\frac{1}{n} I$ as at the end of the proof of Theorem 2.3 and argue similarly.

\section{Real $W^{1, \infty}$-coefficients}

Let $\Omega \subset \mathbb{R}^{d}$ be open, $\theta \in\left[0, \frac{\pi}{2}\right)$ and $\mu, M>0$. Define $\mathcal{S}^{1}(\Omega, \theta, \mu, M$, real) to be the set of all $C \in \mathcal{S}^{1}(\Omega, \theta, \mu, M)$ such that $c_{k l}$ is real valued for all $k, l \in\{1, \ldots, d\}$. If $C \in \mathcal{S}^{1}\left(\Omega, \theta, \mu, M\right.$, real) then $\mathfrak{a}_{C}$ is closable. Moreover, if $\chi \in C_{\mathrm{b}}^{\infty}\left(\mathbb{R}^{d}\right)$ with $\operatorname{supp} \chi \subset \Omega$ then there exists an $a>0$ such that $\left\|M_{\chi} u\right\|_{W^{1,2}\left(\mathbb{R}^{d}\right)} \leq a\|u\|_{D\left(\mathfrak{a}_{C}\right)}$ for all $u \in W^{1,2}\left(\mathbb{R}^{d}\right)$. Hence $M_{\chi}\left(D\left(\overline{\mathfrak{a}_{C}}\right)\right) \subset W^{1,2}\left(\mathbb{R}^{d}\right)$ and $\left\|M_{\chi} u\right\|_{W^{1,2}\left(\mathbb{R}^{d}\right)} \leq$ $a\|u\|_{D\left(\overline{\mathfrak{a}_{C}}\right)}$ for all $u \in D\left(\overline{\mathfrak{a}_{C}}\right)$. In particular, $M_{\chi} S_{t} u \in W^{1,2}\left(\mathbb{R}^{d}\right)$ for all $t>0$ and $u \in L_{2}\left(\mathbb{R}^{d}\right)$.

Throughout the remaining of this paper we set

$$
\tilde{c}_{k l}=c_{k l}+c_{l k}
$$

for all $k, l \in\{1, \ldots, d\}$, whenever $C \in \mathcal{S}(\theta, M)$. 
Lemma 3.1. Let $\Omega \subset \mathbb{R}^{d}$ be open, $\theta \in\left[0, \frac{\pi}{2}\right)$ and $\mu, M>0$. Let $C \in$ $\mathcal{E}^{1}\left(\Omega, \theta, \mu, M\right.$, real). Let $\omega=3 d^{2} M$. Then

$$
\left\|S_{t}^{(\rho)}\right\|_{\infty \rightarrow \infty} \leq e^{\omega\left(|\rho|+\rho^{2}\right) t}
$$

for all $t>0, \rho \in \mathbb{R}$ and $\psi \in \mathcal{D}_{2}$.

Proof. Let $\rho \in \mathbb{R}$ and $\psi \in \mathcal{D}_{2}$. Obviously the form $a_{\rho}$ is real. Integration by parts gives

$$
\begin{aligned}
\mathfrak{a}_{\rho}(u, v)= & \mathfrak{a}(u, v)-\rho \int \sum \tilde{c}_{k l}\left(\partial_{k} u\right)\left(\partial_{l} \psi\right) \bar{v}-\rho \int \sum\left(\partial_{l} c_{k l}\right)\left(\partial_{k} \psi\right) u \bar{v} \\
& -\rho \int \sum c_{k l}\left(\partial_{k} \partial_{l} \psi\right) u \bar{v}-\rho^{2} \int \sum c_{k l}\left(\partial_{k} \psi\right)\left(\partial_{l} \psi\right) u \bar{v}
\end{aligned}
$$

for all $u, v \in W^{1,2}\left(\mathbb{R}^{d}, \mathbb{R}\right)$. Then the lemma follows from [17] Corollary 4.10.

It follows from Lemma 3.1 that the conditions of the next lemma are valid for $p=1$.

Lemma 3.2. Let $\Omega \subset \mathbb{R}^{d}$ be open, $\theta \in\left[0, \frac{\pi}{2}\right), \mu, M>0$ and $p \in[1, \infty)$. Suppose that for all $\varepsilon>0$ and $\chi \in C_{\mathrm{b}}^{\infty}\left(\mathbb{R}^{d}\right)$ with $\chi \neq 0$ and $d\left(\operatorname{supp} \chi, \Omega^{\mathrm{c}}\right)>0$ there exist $a, \omega>0$ such that

$$
\left\|M_{\chi} S_{t}^{(\rho)} u\right\|_{p} \leq a t^{-\frac{d}{2}\left(1-\frac{1}{p}\right)} e^{\omega \rho^{2} t} e^{\varepsilon t}\|u\|_{1}
$$

for all $C \in \mathcal{E}^{1}\left(\Omega, \theta, \mu, M\right.$, real), $t>0, u \in L_{1} \cap L_{2}, \rho \in \mathbb{R}$ and $\psi \in \mathcal{D}_{2}$. Then for all $q \in[p, \infty], v \in(0,1), \varepsilon>0$ and $\chi \in C_{\mathrm{b}}^{\infty}\left(\mathbb{R}^{d}\right)$ with $\frac{1}{p}-\frac{1}{q}<\frac{1-v}{d}, \chi \neq 0$ and $d\left(\operatorname{supp} \chi, \Omega^{\mathrm{c}}\right)>0$ there exist $a, \omega>0$ such that

$$
\begin{aligned}
\left\|M_{\chi} S_{t}^{(\rho)} u\right\|_{q} & \leq a t^{-\frac{d}{2}\left(1-\frac{1}{q}\right)} e^{\omega \rho^{2} t} e^{\varepsilon t}\|u\|_{1} \quad \text { and } \\
\left\|(I-L(h)) M_{\chi} S_{t}^{(\rho)} u\right\|_{q} & \leq a|h|^{v} t^{-\frac{d}{2}\left(1-\frac{1}{q}\right)} t^{-\frac{v}{2}} e^{\omega \rho^{2} t} e^{\varepsilon t}\|u\|_{1}
\end{aligned}
$$

for all $C \in \mathcal{E}^{1}\left(\Omega, \theta, \mu, M\right.$, real), $t>0, u \in L_{1} \cap L_{2}, \rho \in \mathbb{R}, \psi \in \mathcal{D}_{2}$ and $h \in \mathbb{R}^{d}$. Proof. Let $C \in \mathcal{E}^{1}(\Omega, \theta, \mu, M), \varepsilon>0, \rho \in \mathbb{R}, \psi \in \mathcal{D}_{2}, \chi \in C_{\mathrm{b}}^{\infty}\left(\mathbb{R}^{d}\right)$ and suppose $\chi \neq 0$ and $d\left(\operatorname{supp} \chi, \Omega^{\mathrm{c}}\right)>0$. Then

$$
\begin{aligned}
\mathfrak{a}_{\rho}\left(M_{\chi} u, v\right)-\mathfrak{a}_{\rho}\left(u, M_{\chi} v\right)= & -\sum\left(M_{\partial_{l} \chi} \partial_{k} M_{\widetilde{c}_{k l}} u, v\right)+\sum\left(M_{\left(\partial_{l} \chi\right)\left(\partial_{k} c_{k l}\right)} u, v\right) \\
& -\sum\left(M_{\partial_{l} \partial_{k} \chi} M_{c_{k l}} u, v\right) \\
& -\rho \sum\left(M_{\widetilde{c}_{k l}} M_{\partial_{k} \psi} M_{\partial_{l} \chi} u, v\right)
\end{aligned}
$$


for all $u, v \in W^{1,2}\left(\mathbb{R}^{d}\right)$. Note that $\left(\partial_{l} \chi\right)\left(\partial_{k} c_{k l}\right)$ is a bounded function on $\mathbb{R}^{d}$ since supp $\chi \subset \Omega$ and $c_{k l} \in W^{1, \infty}(\Omega)$. Since $C$ is elliptic for all $u, v \in L_{2}\left(\mathbb{R}^{d}\right)$ one deduces

$$
\begin{aligned}
& \left(M_{\chi} S_{t}^{(\rho)} u-S_{t}^{(\rho)} M_{\chi} u, v\right) \\
= & -\int_{0}^{t} \frac{d}{d s}\left(M_{\chi} S_{t-s}^{(\rho)} u, S_{s}^{(\rho) *} v\right) d s \\
= & \int_{0}^{t}\left(-\left(M_{\chi} A_{\rho} S_{t-s}^{(\rho)} u, S_{s}^{(\rho) *} v\right)+\left(M_{\chi} S_{t-s}^{(\rho)} u, A_{\rho}^{*} S_{s}^{(\rho) *} v\right)\right) d s \\
= & \int_{0}^{t} \mathfrak{a}_{\rho}\left(M_{\chi} S_{t-s}^{(\rho)} u, S_{s}^{(\rho) *} v\right)-\mathfrak{a}_{\rho}\left(S_{t-s}^{(\rho)} u, M_{\chi} S_{s}^{(\rho) *} v\right) d s \\
= & -\sum_{k, l=1}^{d} \int_{0}^{t}\left(S_{s}^{(\rho)} M_{\partial_{l} \chi} \partial_{k} M_{\widetilde{c}_{k l}} S_{t-s}^{(\rho)} u, v\right) d s+R
\end{aligned}
$$

where $R$ is the contribution of the last three terms in (3.1), which do not have a derivative. Therefore

$$
\begin{aligned}
M_{\chi^{2}} S_{t}^{(\rho)}-M_{\chi} S_{t}^{(\rho)} M_{\chi} & =M_{\chi}\left[M_{\chi}, S_{t}^{(\rho)}\right] \\
& =-\sum_{k, l=1}^{d} \int_{0}^{t} M_{\chi} S_{s}^{(\rho)} M_{\partial_{k} \chi} \partial_{l} M_{\tilde{c}_{k l}} S_{t-s}^{(\rho)} d s+R^{\prime}
\end{aligned}
$$

with $R^{\prime}$ the contribution of the last three terms in (3.1).

Fix $k, l \in\{1, \ldots, d\}$. Then

$$
\begin{aligned}
& \int_{0}^{t} M_{\chi} S_{s}^{(\rho)} M_{\partial_{k} \chi} \partial_{l} M_{\tilde{c}_{k l}} S_{t-s}^{(\rho)} d s \\
& =\int_{0}^{t / 2} M_{\chi} S_{s}^{(\rho)} M_{\partial_{k} \chi} \partial_{l} M_{\tilde{c}_{k l}} S_{t-s}^{(\rho)} d s+\int_{t / 2}^{t} M_{\chi} S_{s}^{(\rho)} M_{\partial_{k} \chi} \partial_{l} M_{\tilde{c}_{k l}} S_{t-s}^{(\rho)} d s
\end{aligned}
$$

for all $t>0$. By Theorem 2.3 there are $a, \omega>0$ such that

$$
\begin{aligned}
\left\|M_{\chi} S_{s}^{(\rho)} M_{\partial_{k} \chi} \partial_{l}\right\|_{1 \rightarrow q} & \leq a s^{-\frac{d}{2}\left(1-\frac{1}{q}\right)} s^{-1 / 2} e^{\omega \rho^{2} s} e^{\varepsilon s} \quad \text { and } \\
\left\|(I-L(h)) M_{\chi} S_{s}^{(\rho)} M_{\partial_{k} \chi} \partial_{l}\right\|_{1 \rightarrow q} & \leq a|h|^{v} s^{-\frac{d}{2}\left(1-\frac{1}{q}\right)} s^{-\frac{1+v}{2}} e^{\omega \rho^{2} s} e^{\varepsilon s}
\end{aligned}
$$

for all $s>0, \rho \in \mathbb{R}, \psi \in \mathcal{D}_{2}$ and $h \in \mathbb{R}^{d}$. Suppose from now on that $C$ is real valued. Since the matrix of coefficients is real it follows from Lemma 3.1 that there exists an $\omega^{\prime}>0$ such that $\left\|S_{t}^{(\rho)}\right\|_{1 \rightarrow 1} \leq e^{\omega^{\prime} \rho^{2} t} e^{\varepsilon t}$ for all $t>0, \rho \in \mathbb{R}$ and $\psi \in \mathcal{D}_{2}$. 
Then

$$
\begin{aligned}
& \left\|\int_{t / 2}^{t} M_{\chi} S_{s}^{(\rho)} M_{\partial_{k} \chi} \partial_{l} M_{\tilde{c}_{k l}} S_{t-s}^{(\rho)} u d s\right\|_{q} \\
\leq & 2 a M \int_{\frac{t}{2}}^{t} s^{-\frac{d}{2}\left(1-\frac{1}{q}\right)} s^{-1 / 2} e^{\omega \rho^{2} s} e^{\varepsilon s} e^{\omega^{\prime} \rho^{2}(t-s)} e^{\varepsilon(t-s)}\|u\|_{1} d s \\
\leq & 2^{d+1} a M t^{-\frac{d}{2}\left(1-\frac{1}{q}\right)} t^{1 / 2} e^{\left(\omega+\omega^{\prime}\right) \rho^{2} t} e^{\varepsilon t}\|u\|_{1} \\
\leq & 2^{d+1} a \varepsilon^{-1 / 2} M t^{-\frac{d}{2}\left(1-\frac{1}{q}\right)} e^{\left(\omega+\omega^{\prime}\right) \rho^{2} t} e^{2 \varepsilon t}\|u\|_{1}
\end{aligned}
$$

for all $t>0, u \in L_{1} \cap L_{2}, \rho \in \mathbb{R}$ and $\psi \in \mathcal{D}_{2}$. Similarly,

$$
\begin{aligned}
& \left\|\int_{t / 2}^{t}(I-L(h)) M_{\chi} S_{s}^{(\rho)} M_{\partial_{k} \chi} \partial_{l} M_{\tilde{c}_{k l}} S_{t-s}^{(\rho)} u d s\right\|_{q} \\
\leq & 2^{d+1} a \varepsilon^{-1 / 2} M|h|^{\nu} t^{-\frac{d}{2}\left(1-\frac{1}{q}\right)} t^{-\frac{\nu}{2}} e^{\left(\omega+\omega^{\prime}\right) \rho^{2} t} e^{2 \varepsilon t}\|u\|_{1}
\end{aligned}
$$

for all $t>0, u \in L_{1} \cap L_{2}, \rho \in \mathbb{R}, \psi \in \mathcal{D}_{2}$ and $h \in \mathbb{R}^{d}$.

Next we estimate the $L_{1} \rightarrow L_{q}$ norm of the first term in (3.3). There exists a $\tilde{\chi} \in C_{\mathrm{b}}^{\infty}\left(\mathbb{R}^{d}\right)$ such that $\tilde{\chi}(x)=1$ for all $x \in \operatorname{supp} \chi$ and $d\left(\operatorname{supp} \tilde{\chi}, \Omega^{\mathrm{c}}\right)>0$. Then by assumption there exist $a, \omega>0$ such that

$$
\left\|\tilde{\chi} S_{t}^{(\rho)} u\right\|_{p} \leq a t^{-\frac{d}{2}\left(1-\frac{1}{p}\right)} e^{\omega \rho^{2} t} e^{\varepsilon t}\|u\|_{1}
$$

for all $t>0, u \in L_{1} \cap L_{2}, \rho \in \mathbb{R}$ and $\psi \in \mathcal{D}_{2}$. By Theorem 2.3 there are $a^{\prime}, \omega^{\prime}>0$ such that

$$
\begin{aligned}
\left\|M_{\chi} S_{s}^{(\rho)} M_{\partial_{k} \chi} \partial_{l}\right\|_{p \rightarrow q} & \leq a^{\prime} s^{-\frac{d}{2}\left(\frac{1}{p}-\frac{1}{q}\right)} s^{-1 / 2} e^{\omega^{\prime} \rho^{2} s} e^{\varepsilon s} \text { and } \\
\left\|(I-L(h)) M_{\chi} S_{s}^{(\rho)} M_{\partial_{k} \chi} \partial_{l}\right\|_{p \rightarrow q} & \leq a^{\prime}|h|^{\nu} s^{-\frac{d}{2}\left(\frac{1}{p}-\frac{1}{q}\right)} s^{-\frac{1+v}{2}} e^{\omega^{\prime} \rho^{2} s} e^{\varepsilon s}
\end{aligned}
$$

for all $s>0, \rho \in \mathbb{R}, \psi \in \mathcal{D}_{2}$ and $h \in \mathbb{R}^{d}$. Then

$$
\begin{aligned}
& \left\|\int_{0}^{t / 2} M_{\chi} S_{s}^{(\rho)} M_{\partial_{k} \chi} \partial_{l} M_{\tilde{c}_{k l}} S_{t-s}^{(\rho)} u d s\right\|_{q} \\
\leq & \int_{0}^{t / 2}\left\|M_{\chi} S_{s}^{(\rho)} M_{\partial_{k} \chi} \partial_{l}\right\|_{p \rightarrow q}\left\|M_{\tilde{c}_{k l}} M_{\tilde{\chi}} S_{t-s}^{(\rho)} u\right\|_{p} d s \\
\leq & 2 a a^{\prime} M \int_{0}^{\frac{t}{2}} s^{-\frac{d}{2}\left(\frac{1}{p}-\frac{1}{q}\right)} s^{-1 / 2} e^{\omega^{\prime} \rho^{2} s} e^{\varepsilon s}(t-s)^{-\frac{d}{2}\left(1-\frac{1}{q}\right)} e^{\omega \rho^{2}(t-s)} e^{\varepsilon(t-s)}\|u\|_{1} d s \\
\leq & \frac{2^{\frac{d}{2}\left(\frac{1}{p}-\frac{1}{q}\right)+1} a a^{\prime} \varepsilon^{-1 / 2} M}{\frac{1}{2}-\frac{d}{2}\left(\frac{1}{p}-\frac{1}{q}\right)} t^{-\frac{d}{2} g\left(1-\frac{1}{p}\right)} e^{\left(\omega+\omega^{\prime}\right) \rho^{2} t} e^{2 \varepsilon t}\|u\|_{1}
\end{aligned}
$$


for all $t>0, u \in L_{1} \cap L_{2}, \rho \in \mathbb{R}$ and $\psi \in \mathcal{D}_{2}$. Similarly

$$
\begin{aligned}
& \left\|\int_{0}^{t / 2}(I-L(h)) M_{\chi} S_{s}^{(\rho)} M_{\partial_{k} \chi} \partial_{l} M_{\tilde{c}_{k l}} S_{t-s}^{(\rho)} u d s\right\|_{q} \\
\leq & \frac{2^{\frac{d}{2}\left(\frac{1}{p}-\frac{1}{q}\right)+1} a a^{\prime} \varepsilon^{-\frac{1}{2}} M|h|^{v}}{\frac{1-v}{2}-\frac{d}{2}\left(\frac{1}{p}-\frac{1}{q}\right)} t^{-\frac{d}{2}\left(1-\frac{1}{p}\right)} t^{-\frac{v}{2}} e^{\left(\omega+\omega^{\prime}\right) \rho^{2} t} e^{2 \varepsilon t}\|u\|_{1}
\end{aligned}
$$

for all $t>0, u \in L_{1} \cap L_{2}, \rho \in \mathbb{R}, \psi \in \mathcal{D}_{2}$ and $h \in \mathbb{R}^{d}$.

The term $R^{\prime}$ in (3.2) can be estimated similarly. Using Theorem 2.3 to estimate the $L_{1} \rightarrow L_{q}$ norms of $M_{\chi} S_{t}^{(\rho)} M_{\chi}$ and $(I-L(h)) M_{\chi} S_{t}^{(\rho)} M_{\chi}$ one deduces that there are $a, \omega>0$ such that

$$
\begin{aligned}
\left\|M_{\chi^{2}} S_{t}^{(\rho)} u\right\|_{q} & \leq a t^{-\frac{d}{2}\left(1-\frac{1}{q}\right)} e^{\omega \rho^{2} t} e^{\varepsilon t}\|u\|_{1} \quad \text { and } \\
\left\|(I-L(h)) M_{\chi^{2}} S_{t}^{(\rho)} u\right\|_{q} & \leq a|h|^{\nu} t^{-\frac{d}{2}\left(1-\frac{1}{q}\right)} t^{-\frac{\nu}{2}} e^{\omega \rho^{2} t} e^{\varepsilon t}\|u\|_{1}
\end{aligned}
$$

for all $t>0, u \in L_{1} \cap L_{2}, \rho \in \mathbb{R}, \psi \in \mathcal{D}_{2}$ and $h \in \mathbb{R}^{d}$. Then the lemma follows.

Lemma 3.3. Let $\Omega \varsubsetneqq \mathbb{R}^{d}$ be open, $\theta \in\left[0, \frac{\pi}{2}\right), \mu, M>0, v \in(0,1), \varepsilon>0$ and $\chi \in C_{\mathrm{b}}^{\infty}\left(\mathbb{R}^{d}\right)$ with $\chi \neq 0$ and $d\left(\operatorname{supp} \chi, \Omega^{\mathrm{c}}\right)>0$. There there exist $a>0$ and $\omega \geq 0$ such that

$$
\begin{aligned}
\left\|M_{\chi} S_{t}^{(\rho)} u\right\|_{\infty} & \leq a t^{-d / 2} e^{\omega \rho^{2} t} e^{\varepsilon t}\|u\|_{1} \quad \text { and } \\
\left\|(I-L(h)) M_{\chi} S_{t}^{(\rho)} u\right\|_{\infty} & \leq a|h|^{v} t^{-d / 2} t^{-v / 2} e^{\omega \rho^{2} t} e^{\varepsilon t}\|u\|_{1}
\end{aligned}
$$

for all $C \in \mathcal{S}^{1}(\Omega, \theta, \mu, M$, real $), t>0, u \in L_{1} \cap L_{2}, \rho \in \mathbb{R}, \psi \in \mathcal{D}_{2}$ and $h \in \mathbb{R}^{d}$. In particular, $M_{\chi} S_{t} u \in C^{0, v}\left(\mathbb{R}^{d}\right)$ for all $t>0$ and $u \in L_{1}\left(\mathbb{R}^{d}\right)$.

Proof. It follows by induction from Lemmas 3.1 and 3.2 that the current lemma is valid if $\mathcal{S}^{1}(\Omega, \theta, \mu, M$, real $)$ is replaced by $\mathcal{E}^{1}(\Omega, \theta, \mu, M$, real $)$. Then by approximating $C$ by $C+\frac{1}{n} I$ the lemma follows.

Next we turn to derivatives of the semigroup.

Lemma 3.4. Let $u \in W^{1,2}\left(\mathbb{R}^{d}\right), v \in L_{2}\left(\mathbb{R}^{d}\right)$ and $k \in\{1, \ldots, d\}$. Then

$$
\begin{aligned}
\left(\partial_{k} u, v\right) & =\frac{1}{c_{0}} \int_{0}^{\infty} \frac{\left(\left(I-L\left(r e_{k}\right)\right)^{2} u, v\right)}{r} \frac{d r}{r} \\
& =\frac{1}{c_{0}} \int_{0}^{\infty} \frac{\left(I-L\left(r e_{k}\right)\right) u,\left(I-L\left(-r e_{k}\right) v\right)}{r} \frac{d r}{r}
\end{aligned}
$$

where $c_{0}=\int_{0}^{\infty} \frac{\left(1-e^{-r}\right)^{2}}{r} \frac{d r}{r}$. 
Proof. Write $D=\frac{d}{d x_{k}}$, the skew-adjoint operator in $L_{2}\left(\mathbb{R}^{d}\right)$.

Then $\left(\left(I-L\left(r e_{k}\right)\right)^{2} u, v\right)=\left(\left(I-e^{-r D}\right)^{2} u, v\right)$. Now the lemma follows from Fourier theory (or spectral theory).

Lemma 3.5. Let $\Omega \varsubsetneqq \mathbb{R}^{d}$ be open, $\theta \in\left[0, \frac{\pi}{2}\right), \mu, M>0, v \in(0,1), \varepsilon \in(0,1]$ and $\chi \in C_{\mathrm{b}}^{\infty}\left(\mathbb{R}^{d}\right)$ with $\chi \neq 0$ and $d\left(\operatorname{supp} \chi, \Omega^{\mathrm{c}}\right)>0$. Then there exists an $a>0$ such that

$$
\begin{aligned}
\left\|\partial_{m} M_{\chi} S_{t}\right\|_{1 \rightarrow \infty} & \leq a t^{-d / 2} t^{-1 / 2} e^{\varepsilon t} \text { and } \\
\left\|(I-L(h)) \partial_{m} M_{\chi} S_{t}\right\|_{1 \rightarrow \infty} & \leq a t^{-d / 2} t^{-1 / 2}\left(\frac{|h|}{\sqrt{t}}\right)^{v} e^{\varepsilon t}
\end{aligned}
$$

for all $C \in \mathcal{E}^{1}(\Omega, \theta, \mu, M$, real $), t>0, m \in\{1, \ldots, d\}$ and $h \in \mathbb{R}^{d}$.

Proof. We only prove the second estimate, the proof of the first one is similar. We argue as in the proof of Lemma 3.2 and use the commutator (3.2). There exists a $\tilde{\chi} \in C_{\mathrm{b}}^{\infty}\left(\mathbb{R}^{d}\right)$ such that $\tilde{\chi}(x)=1$ for all $x \in \operatorname{supp} \chi$ and $d\left(\operatorname{supp} \tilde{\chi}, \Omega^{\mathrm{c}}\right)>0$. Now we have

$$
\begin{aligned}
& (I-L(h)) \partial_{m}\left(M_{\chi^{2}} S_{t}-M_{\chi} S_{t} M_{\chi}\right) \\
& =-\sum_{k, l=1}^{d} \int_{0}^{t}(I-L(h)) \partial_{m} M_{\chi} S_{s} M_{\partial_{k} \chi} \partial_{l} M_{\tilde{c}_{k l}} M_{\tilde{\chi}} S_{t-s} d s+R,
\end{aligned}
$$

where $R$ is the contribution of the second and third term in (3.1), which do not have a derivative. Note that the last term in (3.1) vanishes since $\rho=0$. Again we split the integral in two parts. Fix $k, l \in\{1, \ldots, d\}$. Then

$$
\begin{aligned}
\left\|\int_{t / 2}^{t}(I-L(h)) \partial_{m} M_{\chi} S_{s} M_{\partial_{k} \chi} \partial_{l} M_{\tilde{c}_{k l}} M_{\tilde{\chi}} S_{t-s} d s\right\|_{1 \rightarrow \infty} \\
\quad \leq \int_{t / 2}^{t}\left\|(I-L(h)) \partial_{m} M_{\chi} S_{s} M_{\partial_{k} \chi} \partial_{l}\right\|_{1 \rightarrow \infty}\left\|M_{\tilde{c}_{k l}} M_{\tilde{\chi}} S_{t-s}\right\|_{1 \rightarrow 1} d s \\
\quad \leq a t^{-d / 2}\left(\frac{|h|}{\sqrt{t}}\right)^{v} e^{\varepsilon t}
\end{aligned}
$$

for a suitable $a>0$, by the estimates of Theorem 2.3 and Lemma 3.1. 
For the integral over $\left(0, \frac{t}{2}\right)$ we use Lemma 3.4 and write

$$
\begin{aligned}
&\left\|\int_{0}^{t / 2}(I-L(h)) \partial_{m} M_{\chi} S_{s} M_{\partial_{k} \chi} \partial_{l} M_{\tilde{c}_{k l}} M_{\tilde{\chi}} S_{t-s} d s\right\|_{1 \rightarrow \infty} \\
&= c_{0}^{-1} \sup _{\substack{u, v \in W^{1,2}\left(\mathbb{R}^{d}\right) \cap L_{1}\left(\mathbb{R}^{d}\right) \\
\|u\|_{1},\|v\|_{1} \leq 1}} \mid \int_{0}^{t / 2} \int_{0}^{\infty}\left(\left(I-L\left(r e_{l}\right)\right) M_{\tilde{c}_{k l}} M_{\tilde{\chi}} S_{t-s} u,\right. \\
&\left.\leq c_{0}^{-1} \sup _{\substack{u, v \in W^{1,2}\left(\mathbb{R}^{d}\right) \cap L_{1}\left(\mathbb{R}^{d}\right) \\
\|u\|_{1},\|v\|_{1} \leq 1}} \int_{0}^{t / 2} \int_{0}^{\infty} \|\left(I-L\left(r e_{l}\right)\right) M_{\tilde{c}_{k l} \chi} S_{s}^{*} M_{\chi} \partial_{m}(I-L(-h)) v\right) \frac{d r}{r^{2}} d s \mid \\
& \times \times\left(I-L\left(-r e_{l}\right)\right) M_{\partial_{k} \chi} S_{s}^{*} M_{\chi} \partial_{m}(I-L(-h)) v \|_{1} \frac{d r}{r^{2}} d s,
\end{aligned}
$$

where $c_{0}$ is as in Lemma 3.4. Next split the integral over $(0, \infty)$ in two parts: $(0,1]$ and $[1, \infty)$. There exist $v_{1}, v_{2} \in(0,1)$ such that $v_{2}+v<1$ and $v_{1}+v_{2}>1$. By Theorem 2.3 and Lemma 3.3 there exists an $a>0$ such that

$$
\begin{aligned}
&\left\|\left(I-L\left(h_{1}\right)\right) M_{\tilde{c}_{k l}} M_{\tilde{\chi}} S_{s}\right\|_{1 \rightarrow \infty} \leq a s^{-d / 2}\left(\frac{\left|h_{1}\right|}{\sqrt{s}}\right)^{\nu_{1}} e^{\varepsilon s}, \\
&\left\|M_{\tilde{c}_{k l}} M_{\tilde{\chi}} S_{s}\right\|_{1 \rightarrow \infty} \leq a s^{-d / 2} e^{\varepsilon s}, \\
&\left\|\left(I-L\left(h_{1}\right)\right) M_{\partial_{k} \chi} S_{s}^{*} M_{\chi} \partial_{m}\left(I-L\left(h_{2}\right)\right)\right\|_{1 \rightarrow 1} \leq a s^{-1 / 2}\left(\frac{\left|h_{1}\right|}{\sqrt{s}}\right)^{\nu_{2}}\left(\frac{\left|h_{2}\right|}{\sqrt{s}}\right)^{v} e^{\varepsilon s} \text { and } \\
&\left\|M_{\partial_{k} \chi} S_{s}^{*} M_{\chi} \partial_{m}\left(I-L\left(h_{2}\right)\right)\right\|_{1 \rightarrow 1} \leq a s^{-1 / 2}\left(\frac{\left|h_{2}\right|}{\sqrt{s}}\right)^{v} e^{\varepsilon s}
\end{aligned}
$$

for all $s>0$ and $h_{1}, h_{2} \in \mathbb{R}^{d}$. Let $u, v \in W^{1,2}\left(\mathbb{R}^{d}\right) \cap L_{1}\left(\mathbb{R}^{d}\right)$. Then

$$
\begin{aligned}
\int_{0}^{t / 2} & \int_{0}^{1}\left\|\left(I-L\left(r e_{l}\right)\right) M_{\tilde{c}_{k l}} M_{\tilde{\chi}} S_{t-s} u\right\|_{\infty}\left\|\left(I-L\left(-r e_{l}\right)\right) M_{\partial_{k} \chi} S_{s}^{*} M_{\chi} \partial_{m}(I-L(-h)) v\right\|_{1} \\
& \times \frac{d r}{r^{2}} d s \\
\leq & a^{2} \int_{0}^{t / 2} \int_{0}^{1}(t-s)^{-d / 2}\left(\frac{r}{\sqrt{t-s}}\right)^{\nu_{1}} e^{\varepsilon(t-s)} s^{-1 / 2}\left(\frac{|h|}{\sqrt{s}}\right)^{v}\left(\frac{r}{\sqrt{s}}\right)^{\nu_{2}} e^{\varepsilon s}\|u\|_{1}\|v\|_{1} \\
& \times \frac{d r}{r^{2}} d s \\
= & a^{\prime} t^{-d / 2} t^{-1 / 2}\left(\frac{|h|}{\sqrt{t}}\right)^{v} e^{\varepsilon t} t^{\frac{2-\left(v_{1}+v_{2}\right)}{2}}\|u\|_{1}\|v\|_{1} \\
\leq & a^{\prime} \varepsilon^{-\frac{2-\left(\nu_{1}+v_{2}\right)}{2}} t^{-d / 2} t^{-1 / 2}\left(\frac{|h|}{\sqrt{t}}\right)^{v} e^{2 \varepsilon t}\|u\|_{1}\|v\|_{1}
\end{aligned}
$$


for a suitable $a^{\prime}>0$. Similarly, since left translations are isometries, one deduces

$$
\begin{aligned}
& \int_{0}^{t / 2} \int_{1}^{\infty}\left\|\left(I-L\left(r e_{l}\right)\right) M_{\tilde{c}_{k l}} M_{\tilde{\chi}} S_{t-s} u\right\|_{\infty}\left\|\left(I-L\left(-r e_{l}\right)\right) M_{\partial_{k} \chi} S_{s}^{*} M_{\chi} \partial_{m}(I-L(-h)) v\right\|_{1} \\
& \quad \times \frac{d r}{r^{2}} d s \\
& \leq 4 a^{2} \int_{0}^{t / 2} \int_{1}^{\infty}(t-s)^{-d / 2} e^{\varepsilon(t-s)} s^{-1 / 2}\left(\frac{|h|}{\sqrt{s}}\right)^{v} e^{\varepsilon s}\|u\|_{1}\|v\|_{1} \frac{d r}{r^{2}} d s \\
& =a^{\prime \prime} t^{-d / 2} t^{1 / 2}\left(\frac{|h|}{\sqrt{t}}\right)^{v} e^{\varepsilon t}\|u\|_{1}\|v\|_{1} \\
& \leq a^{\prime \prime} \varepsilon^{-1} t^{-d / 2} t^{-1 / 2}\left(\frac{|h|}{\sqrt{t}}\right)^{v} e^{2 \varepsilon t}\|u\|_{1}\|v\|_{1}
\end{aligned}
$$

for a suitable $a^{\prime \prime}>0$. As before, the contribution of the term $R$ in (3.4) can be estimated similarly and we leave the rest of the proof to the reader.

We next replace $\mathcal{E}^{1}\left(\Omega, \theta, \mu, M\right.$, real) by $\mathcal{S}^{1}(\Omega, \theta, \mu, M$, real) in Lemma 3.5.

Lemma 3.6. Let $\Omega \varsubsetneqq \mathbb{R}^{d}$ be open, $\theta \in\left[0, \frac{\pi}{2}\right), \mu, M>0, v \in(0,1), \varepsilon \in(0,1]$ and $\chi \in C_{\mathrm{b}}^{\infty}\left(\mathbb{R}^{d}\right)$ with $\chi \neq 0$ and $d\left(\operatorname{supp} \chi, \Omega^{\mathrm{c}}\right)>0$. Then there exists an $a>0$ such that

$$
\begin{gathered}
\left\|\partial_{m} M_{\chi} S_{t}\right\|_{1 \rightarrow \infty} \leq a t^{-d / 2} t^{-1 / 2} e^{\varepsilon t} \text { and } \\
\left\|(I-L(h)) \partial_{m} M_{\chi} S_{t}\right\|_{1 \rightarrow \infty} \leq a t^{-d / 2} t^{-1 / 2}\left(\frac{|h|}{\sqrt{t}}\right)^{v} e^{\varepsilon t}
\end{gathered}
$$

for all $C \in \mathcal{S}^{1}(\Omega, \theta, \mu, M$, real $), t>0, m \in\{1, \ldots, d\}$ and $h \in \mathbb{R}^{d}$. In particular, $M_{\chi} S_{t} u \in W^{1+v, \infty}\left(\mathbb{R}^{d}\right)$ for all $t>0$ and $u \in L_{1}\left(\mathbb{R}^{d}\right)$.

Proof. By Lemma 3.5 there exists an $a>0$ such that

$$
\left\|\partial_{m} M_{\chi} S_{t}\right\|_{1 \rightarrow \infty} \leq a t^{-d / 2} t^{-1 / 2} e^{\varepsilon t}
$$

for all $C \in \mathcal{E}^{1}(\Omega, \theta, \mu, M+1$, real $), t>0$ and $m \in\{1, \ldots, d\}$. Fix $C \in$ $\mathcal{S}^{1}(\Omega, \theta, \mu, M$, real $)$. For all $n \in \mathbb{N}$ define $C^{(n)}=C+\frac{1}{n} I$. Let $t>0, m \in$ $\{1, \ldots, d\}$ and $u, v \in C_{c}^{\infty}\left(\mathbb{R}^{d}\right)$. Then it follows from (3.5) that

$$
\left|\left(M_{\chi} S_{t}^{C^{(n)}} u, \partial_{m} v\right)\right| \leq a t^{-d / 2} t^{-1 / 2} e^{\varepsilon t}\|u\|_{1}\|v\|_{1}
$$

for all $n \in \mathbb{N}$. Since $\lim S_{t}^{C^{(n)}}=S_{t}^{C}$ strongly in $L_{2}\left(\mathbb{R}^{d}\right)$ by [3] Corollary 3.9 it follows that

$$
\left|\left(M_{\chi} S_{t}^{C} u, \partial_{m} v\right)\right| \leq a t^{-d / 2} t^{-1 / 2} e^{\varepsilon t}\|u\|_{1}\|v\|_{1}
$$

for all $u, v \in C_{c}^{\infty}\left(\mathbb{R}^{d}\right)$. By continuity it then follows that (3.6) is valid for all $u \in L_{1}\left(\mathbb{R}^{d}\right)$ and $v \in C_{c}^{\infty}\left(\mathbb{R}^{d}\right)$. This implies that $M_{\chi} S_{t}^{C} u \in W^{1, \infty}\left(\mathbb{R}^{d}\right)$ and the first estimate of the lemma is valid. The second one follows similarly. 
It follows from Lemma 3.3 that for each $t>0$ there exists a measurable function $L_{t}: \mathbb{R}^{d} \times \mathbb{R}^{d} \rightarrow \mathbb{R}$ such that $L_{t}$ is a kernel of $M_{\chi} S_{t}$ and $L_{t}$ satisfies Gaussian bounds. But then it is unclear whether $x \mapsto L_{t}(x, y)$ is Hölder continuous or differentiable for some $y \in \mathbb{R}^{d}$. Even worse, there is no reason that the combined map $(t, x, y) \mapsto L_{t}(x, y)$ from $(0, \infty) \times \mathbb{R}^{d} \times \mathbb{R}^{d}$ into $\mathbb{R}$ is measurable. In order to circumvent this measurability problem with the uncountable many null sets, we first obtain a measurable map on $(0, \infty) \times \mathbb{R}^{d} \times \mathbb{R}^{d}$ for the kernels of $M_{\chi} S_{t}$ and its derivatives $\partial_{m} M_{\chi} S_{t}$ and then consider continuity and differential properties in Theorem 3.9.

Proposition 3.7. Let $\Omega \varsubsetneqq \mathbb{R}^{d}$ be open, $\theta \in\left[0, \frac{\pi}{2}\right), \mu, M>0$ and $\chi \in C_{\mathrm{b}}^{\infty}\left(\mathbb{R}^{d}\right)$ with $\chi \neq 0, \varepsilon>0$ and $d\left(\operatorname{supp} \chi, \Omega^{\mathrm{c}}\right)>0$. Then there exist $a, b>0$ such that for all $C \in \mathcal{S}^{1}(\Omega, \theta, \mu, M$, real) and $m \in\{1, \ldots, d\}$ there exist measurable functions $(t, x, y) \mapsto K_{t}(x, y)$ and $(t, x, y) \mapsto K_{t}^{(m)}(x, y)$ from $(0, \infty) \times \mathbb{R}^{d} \times \mathbb{R}^{d}$ into $\mathbb{R}$ such that $K_{t}$ is a kernel of the operator $M_{\chi} S_{t}$ and $K_{t}^{(m)}$ is a kernel of the operator $\partial_{m} M_{\chi} S_{t}$ for all $t>0$. Moreover,

$$
\begin{aligned}
\left|K_{t}(x, y)\right| & \leq a t^{-d / 2} e^{\varepsilon t} e^{-b \frac{|x-y|^{2}}{t}} \text { and } \\
\left|K_{t}^{(m)}(x, y)\right| & \leq a t^{-d / 2} t^{-1 / 2} e^{\varepsilon t} e^{-b \frac{|x-y|^{2}}{t}}
\end{aligned}
$$

for all $t>0$ and $(x, y) \in \mathbb{R}^{d} \times \mathbb{R}^{d}$.

Proof. Let $a, \omega$ be as in Lemma 3.3 (with $v=\frac{1}{2}$ ). Let $C \in \mathcal{S}^{1}(\Omega, \theta, \mu, M$, real). Then $M_{\chi} S_{t}$ is a continuous operator from $L_{2}\left(\mathbb{R}^{d}\right)$ into $L_{\infty}\left(\mathbb{R}^{d}\right)$ for all $t>0$ by Lemma 2.5. Moreover, $z \mapsto S_{z}$ is a holomorphic contraction semigroup on $\Sigma_{\theta_{\mathfrak{a}}}^{\circ}$. Hence $M_{\chi} S_{z}$ maps $L_{2}\left(\mathbb{R}^{d}\right)$ continuously into $L_{\infty}\left(\mathbb{R}^{d}\right)$ for all $z \in \Sigma_{\theta_{\mathfrak{a}}}^{\circ}$ and $z \mapsto M_{\chi} S_{z} u$ is holomorphic for all $u \in L_{2}\left(\mathbb{R}^{d}\right)$. It follows from the discussion after Definition 1.8 in [2] and [2] Theorem 3.1 that there exists a measurable function $(z, x, y) \mapsto K_{z}(x, y)$ from $\Sigma_{\theta_{\mathfrak{a}}}^{\circ} \times \mathbb{R}^{d} \times \mathbb{R}^{d}$ into $\mathbb{C}$ such that $z \mapsto K_{z}(x, y)$ is holomorphic for all $x, y \in \mathbb{R}^{d}$ and $K_{z}$ is the kernel of $M_{\chi} S_{z}$ for all $z \in \Sigma_{\theta_{\mathfrak{a}}}^{\circ}$. In particular, $K_{t}$ is a kernel of $M_{\chi} S_{t}$ for all $t>0$. By the usual minimising argument the estimates of Lemma 3.3 give Gaussian bounds for the kernel $K_{t}$ for each $t>0$. Precisely, for each $t>0$ one has $\left|K_{t}\right| \leq G_{t}$ a.e. on $\mathbb{R}^{d} \times \mathbb{R}^{d}$, where $G_{t}(x, y)=a t^{-d / 2} e^{\varepsilon t} e^{-b \frac{|x-y|^{2}}{t}}$ for all $t>0$ and $x, y \in \mathbb{R}^{d}$, where $b$ depends only on $\omega$ and $d$. Obviously $(t, x, y) \mapsto G_{t}(x, y)$ is a continuous function from $(0, \infty) \times \mathbb{R}^{d} \times \mathbb{R}^{d}$ into $\mathbb{R}$, therefore it is measurable. Then $\left(-G_{t}\right) \vee K_{t} \wedge G_{t}$ is also a kernel of $M_{\chi} S_{t}$ for each $t>0$. Now the proposition follows for $M_{\chi} S_{t}$ with $K_{t}$ replaced by $\left(-G_{t}\right) \vee K_{t} \wedge G_{t}$.

The argument for $\partial_{m} M_{\chi} S_{t}$ is similar.

The next lemma is also valid for complex coefficients. The complex version will be used in the proof of Proposition 4.11. 
Lemma 3.8. Let $\Omega \varsubsetneqq \mathbb{R}^{d}$ be open, $\theta \in\left[0, \frac{\pi}{2}\right), \mu, M>0, C \in \mathcal{S}^{1}(\Omega, \theta, \mu, M)$ and $\chi \in C_{\mathrm{b}}^{\infty}\left(\mathbb{R}^{d}\right)$ with $\chi \neq 0$ and $d\left(\operatorname{supp} \chi, \Omega^{\mathrm{c}}\right)>0$. Let $\tilde{\chi} \in C_{\mathrm{b}}^{\infty}\left(\mathbb{R}^{d}\right)$ be such that $d\left(\operatorname{supp} \tilde{\chi}, \Omega^{\mathrm{c}}\right)>0$ and $\tilde{\chi}(x)=1$ for all $x \in \operatorname{supp} \chi$. Then

$$
\begin{aligned}
M_{\chi}^{2} S_{t}= & M_{\chi} S_{t} M_{\chi}-\sum_{k, l=1}^{d} \int_{0}^{t} M_{\chi} S_{s} M_{\partial_{k} \chi} \partial_{l} M_{\tilde{c}_{k l}} M_{\tilde{\chi}} S_{t-s} d s \\
& +\sum_{k, l=1}^{d} \int_{0}^{t} M_{\chi} S_{s}\left(M_{\partial_{l} \tilde{c}_{k l}} M_{\partial_{k} \chi} S_{t-s}-M_{c_{k l}} M_{\partial_{k} \partial_{l} \chi} S_{t-s}\right) d s
\end{aligned}
$$

for all $t>0$, where the operators act in $L_{2}\left(\mathbb{R}^{d}\right)$.

Proof. It follows from Lemma 2.1(d) that the integrals on the right hand side of (3.7) are convergent.

If $C \in \mathcal{E}^{1}(\Omega, \theta, \mu, M)$ then (3.7) follows from (3.2). For all $n \in \mathbb{N}$ let $C_{n}=$ $C+\frac{1}{n} I$. Then $C_{n} \in \mathcal{E}^{1}(\Omega, \theta, \mu, M+1)$. We use (3.7) with respect to $C^{(n)}$. Write $S=S^{C}$ and $S^{(n)}=S^{C_{n}}$. It follows from Lemma 2.1(d) that there exist $a, \omega>0$ such that

$$
\left\|M_{\chi} S_{s}^{(n)} M_{\partial_{k} \chi} \partial_{l}\right\|_{2 \rightarrow 2} \leq a s^{-1 / 2} e^{\omega s}
$$

for all $s>0$ and $n \in \mathbb{N}$. Obviously $\left\|S_{s}^{(n)}\right\|_{2 \rightarrow 2} \leq 1$ for all $s>0$ and $n \in \mathbb{N}$.

Let $u, v \in L_{2}\left(\mathbb{R}^{d}\right)$. Then $\lim _{n \rightarrow \infty} S_{s}^{(n)} u=S_{s} u$ in $L_{2}\left(\mathbb{R}^{d}\right)$ for all $s \in(0, t]$ by [3, Corollary 3.9]. Note that $M_{\tilde{\chi}} S_{s} u \in W^{1,2}\left(\mathbb{R}^{d}\right)$ for all $s>0$. Let $k, l \in$ $\{1, \ldots, d\}$. Then

$$
\begin{aligned}
& \left|\left(M_{\chi} S_{s}^{(n)} M_{\partial_{k} \chi} \partial_{l} M_{\tilde{c}^{(n)} k l} M_{\tilde{\chi}} S_{t-s}^{(n)} u, v\right)-\left(M_{\chi} S_{s} M_{\partial_{k} \chi} \partial_{l} M_{\tilde{c}_{k l}} M_{\tilde{\chi}} S_{t-s} u, v\right)\right| \\
\leq & \frac{1}{n}\left|\left(M_{\chi} S_{s}^{(n)} M_{\partial_{k} \chi} \partial_{l} S_{t-s}^{(n)} u, v\right)\right| \\
& +\left|\left(M_{\tilde{c}_{k l}}\left(S_{t-s}^{(n)} u-S_{t-s} u\right), \partial_{l} M_{\partial_{k} \chi} S_{s}^{(n)^{*}} M_{\chi} v\right)\right| \\
& +\left|\left(\partial_{l} M_{\tilde{c}_{k l}} M_{\tilde{\chi}} S_{t-s} u, M_{\partial_{k} \chi}\left(S_{s}^{(n)^{*}} M_{\chi} v-S_{s}^{*} M_{\chi} v\right)\right)\right|
\end{aligned}
$$

for all $s \in(0, t)$ and $n \in \mathbb{N}$. So

$$
\lim _{n \rightarrow \infty}\left(M_{\chi} S_{s}^{(n)} M_{\partial_{k} \chi} \partial_{l} M_{c^{(n)} k l} M_{\widetilde{\chi}} S_{t-s}^{(n)} u, v\right)=\left(M_{\chi} S_{s} M_{\partial_{k} \chi} \partial_{l} M_{\tilde{c}_{k l}} M_{\widetilde{\chi}} S_{t-s} u, v\right)
$$

for all $s \in(0, t)$. Moreover,

$$
\left|\left(M_{\chi} S_{s}^{(n)} M_{\partial_{k} \chi} \partial_{l} M_{c^{(n)} k l} M_{\widetilde{\chi}} S_{t-s}^{(n)} u, v\right)\right| \leq a s^{-1 / 2} e^{\omega s}\|u\|_{2}\|v\|_{2}
$$

for all $s \in(0, t)$ and $n \in \mathbb{N}$. So

$$
\begin{aligned}
& \lim _{n \rightarrow \infty} \int_{0}^{t}\left(M_{\chi} S_{s}^{(n)} M_{\partial_{k} \chi} \partial_{l} M_{c^{(n)}{ }_{k l}} M_{\tilde{\chi}} S_{t-s}^{(n)} u, v\right) d s \\
& =\int_{0}^{t}\left(M_{\chi} S_{s} M_{\partial_{k} \chi} \partial_{l} M_{\tilde{c}_{k l}} M_{\widetilde{\chi}} S_{t-s} u, v\right) d s
\end{aligned}
$$

by the Lebesgue dominated convergence theorem. 
One can treat similarly the last term on the right hand side of (3.7) and the lemma follows.

The next theorem is a uniform version of Theorem 1.2.

Theorem 3.9. Let $\Omega \varsubsetneqq \mathbb{R}^{d}$ be open, $\theta \in\left[0, \frac{\pi}{2}\right), \mu, M>0, v \in(0,1), \kappa>0$, $\tau \in[0,1), \varepsilon \in(0,1]$ and $\chi \in C_{\mathrm{b}}^{\infty}\left(\mathbb{R}^{d}\right)$ with $\chi \neq 0$ and $d\left(\operatorname{supp} \chi, \Omega^{\mathrm{c}}\right)>0$. Then there exist $a, b>0$ such that for all $C \in \mathcal{S}^{1}(\Omega, \theta, \mu, M$, real) there exists $a$ measurable function $(t, x, y) \mapsto K_{t}(x, y)$ from $(0, \infty) \times \mathbb{R}^{d} \times \mathbb{R}^{d}$ into $\mathbb{R}$ such that the following is valid.

(a) For all $t>0$ the function $K_{t}$ is a kernel of $M_{\chi} S_{t}$.

(b) For all $t>0$ and $y \in \mathbb{R}^{d}$ the function $x \mapsto K_{t}(x, y)$ is continuously differentiable on $\mathbb{R}^{d}$.

(c) The function $t \mapsto\left(\partial_{x}^{\alpha} K_{t}\right)(x, y)$ is continuous for all $x, y \in \mathbb{R}^{d}$ and multiindex $\alpha$ with $|\alpha| \leq 1$.

(d) For every multi-index $\alpha$ with $|\alpha| \leq 1$ one has

$$
\left|\left(\partial_{x}^{\alpha} K_{t}\right)(x, y)\right| \leq a t^{-d / 2} t^{-|\alpha| / 2} e^{\varepsilon t} e^{-b \frac{|x-y|^{2}}{t}}
$$

and

$$
\begin{aligned}
& \left|\left(\partial_{x}^{\alpha} K_{t}\right)(x+h, y)-\left(\partial_{x}^{\alpha} K_{t}\right)(x, y)\right| \\
& \leq a t^{-d / 2} t^{-|\alpha| / 2}\left(\frac{|h|}{|x-y|+\sqrt{t}}\right)^{v} e^{\varepsilon t} e^{-b \frac{|x-y|^{2}}{t}}
\end{aligned}
$$

for all $t>0$ and $x, y, h \in \mathbb{R}^{d}$ with $|h| \leq \tau|x-y|+\kappa \sqrt{t}$.

(e) $\left(\partial^{\alpha} M_{\chi} S_{t} u\right)(x)=\int_{\mathbb{R}^{d}}\left(\partial_{x}^{\alpha} K_{t}\right)(x, y) u(y) d y$ for all $t>0, u \in L_{1}\left(\mathbb{R}^{d}\right), x \in$ $\mathbb{R}^{d}$ and multi-index $\alpha$ with $|\alpha| \leq 1$.

Proof. Without loss of generality we may prove the proposition with $M_{\chi} S_{t}$ replaced by $M_{\chi}^{2} S_{t}$. We consider each of the operators in the terms on the right hand side of (3.7). It is possible to differentiate all terms in (3.7) at least once in $L_{2}$-sense. Let $\alpha$ be a multi-index with $|\alpha| \leq 1$. Clearly $\partial^{\alpha} M_{\chi} S_{t} M_{\chi}$ has a kernel satisfying the stated requirements by Theorem 2.3. Let $k, l \in\{1, \ldots, d\}$. For all $t>0$ consider the operator

$$
\int_{0}^{t} \partial^{\alpha} M_{\chi} S_{s} M_{\partial_{k} \chi} \partial_{l} M_{\tilde{c}_{k l}} M_{\tilde{\chi}} S_{t-s} d s .
$$

By Theorem 2.3 there exist a continuous function $(t, x, y) \mapsto L_{t}^{(1, \alpha)}(x, y)$ from $(0, \infty) \times \mathbb{R}^{d} \times \mathbb{R}^{d}$ into $\mathbb{R}$ and suitable constants $a_{1}, b_{1}>0$ such that for all $t>0$ the function $L_{t}^{(1, \alpha)}$ is a kernel of the operator $\partial^{\alpha} M_{\chi} S_{t} M_{\partial_{k} \chi}$,

$$
\begin{aligned}
\left|L_{t}^{(1, \alpha)}(x, y)\right| & \leq a_{1} t^{-d / 2} t^{-|\alpha| / 2} e^{\varepsilon t} e^{-b_{1} \frac{|x-y|^{2}}{t}} \text { and } \\
\left|L_{t}^{(1, \alpha)}(x+h, y)-L_{t}^{(1, \alpha)}(x, y)\right| & \leq a_{1} t^{-d / 2} t^{-|\alpha| / 2}\left(\frac{|h|}{\sqrt{t}}\right)^{v} e^{\varepsilon t} e^{-b_{1} \frac{|x-y|^{2}}{t}}
\end{aligned}
$$


for all $t>0$ and $x, y, h \in \mathbb{R}^{d}$ with $|h| \leq \frac{1}{2}|x-y|+\sqrt{t}$. Next, by Proposition 3.7 there exist a measurable function $(t, x, y) \mapsto L_{t}^{(2)}(x, y)$ from $(0, \infty) \times \mathbb{R}^{d} \times \mathbb{R}^{d}$ into $\mathbb{R}$ and suitable constants $a_{2}, b_{2}>0$ such that for all $t>0$ the function $L_{t}^{(2)}$ is a kernel of the operator $\partial_{l} M_{\tilde{c}_{k l}} M_{\tilde{\chi}} S_{t}$ and

$$
\left|L_{t}^{(2)}(x, y)\right| \leq a_{2} t^{-d / 2} t^{-1 / 2} e^{\varepsilon t} e^{-b_{2} \frac{|x-y|^{2}}{t}}
$$

for all $t>0$ and $x, y \in \mathbb{R}^{d}$. Define the function $(t, x, y) \mapsto L_{t}^{(3, \alpha)}(x, y)$ from $(0, \infty) \times \mathbb{R}^{d} \times \mathbb{R}^{d}$ into $\mathbb{R}$ by

$$
L_{t}^{(3, \alpha)}(x, y)=\int_{0}^{t} \int_{\mathbb{R}^{d}} L_{s}^{(1, \alpha)}(x, z) L_{t-s}^{(2)}(z, y) d z d s
$$

Since the convolution of two Gaussians is a Gaussian, it follows that the integral is convergent and $L^{(3, \alpha)}$ has appropriate Gaussian bounds. Moreover, $(t, x, y) \mapsto$ $L_{t}^{(3, \alpha)}(x, y)$ is measurable and $L_{t}^{(3, \alpha)}$ is a kernel of the operator (3.9) for all $t>0$. Define $L_{t}^{(3)}=L^{(3, \beta)}$ if $|\beta|=0$. It is an elementary exercise in integration theory to prove that $x \mapsto L_{t}^{(3, \alpha)}(x, y)$ is continuous for all $t>0$ and $y \in \mathbb{R}^{d}$, that $t \mapsto L_{t}^{(3, \alpha)}(x, y)$ is continuous for all $x, y \in \mathbb{R}^{d}$ and that for all $t>0$ and $y \in \mathbb{R}^{d}$ the function $x \mapsto L_{t}^{(3)}(x, y)$ is differentiable and $\left(\partial_{x}^{\alpha} L_{t}^{(3)}\right)(x, y)=L_{t}^{(3, \alpha)}(x, y)$ for all $x \in \mathbb{R}^{d}$.

The last term in (3.7) can be treated in a similar way and Statements (a)-(c) and the first part of Statement (d) follow. Let $K$ be the so obtained kernel.

Next, let $t>0$ and $u \in L_{1}\left(\mathbb{R}^{d}\right)$. Since $(x, y) \mapsto\left(\partial_{x}^{\alpha} K_{t}\right)(x, y)$ is a kernel of $\partial^{\alpha} M_{\chi} S_{t}$ it follows that $\left(\partial^{\alpha} M_{\chi} S_{t} u\right)(x)=\int_{\mathbb{R}^{d}}\left(\partial_{x}^{\alpha} K_{t}\right)(x, y) u(y) d y$ for a.e. $x \in \mathbb{R}^{d}$. Hence this is valid for all $x \in \mathbb{R}^{d}$ since $\partial^{\alpha} M_{\chi} S_{t} u$ is continuous by Lemmas 3.3 and 3.6. This proves Statement (e).

Finally we establish the Hölder Gaussian bounds of Statement (d). Set $\tilde{v}=$ $\frac{1}{2}(1+v)$. By Lemma 3.6 there exists an $a_{1}>0$, depending only on $\Omega, \theta, \mu, M, v$, $\varepsilon$ and $\chi$, such that

$$
\left\|(I-L(h)) \partial_{m} M_{\chi} S_{t}\right\|_{1 \rightarrow \infty} \leq a_{1} t^{-d / 2} t^{-1 / 2}\left(\frac{|h|}{\sqrt{t}}\right)^{\tilde{v}} e^{\varepsilon t}
$$

for all $t>0, m \in\{1, \ldots, d\}$ and $h \in \mathbb{R}^{d}$. Let $x, h \in \mathbb{R}^{d}, t>0$ and $m \in\{1, \ldots, d\}$. Then it follows from Statement (e) that

$$
\begin{aligned}
\left|\int\left(\left(\partial_{x, m} K_{t}\right)(x, y)-\left(\partial_{x, m} K_{t}\right)(x-h, y)\right) u(y) d y\right| & =\left|\left((I-L(h)) \partial_{m} M_{\chi} S_{t} u\right)(x)\right| \\
& \leq a_{1} t^{-d / 2} t^{-1 / 2}\left(\frac{|h|}{\sqrt{t}}\right)^{\tilde{v}} e^{\varepsilon t}\|u\|_{1}
\end{aligned}
$$


for all $u \in L_{1}\left(\mathbb{R}^{d}\right)$. Hence there exists a null set $N_{x, h, t, m} \subset \mathbb{R}^{d}$ such that

$$
\left|\left(\partial_{x, m} K_{t}\right)(x, y)-\left(\partial_{x, m} K_{t}\right)(x-h, y)\right| \leq a_{1} t^{-d / 2} t^{-1 / 2}\left(\frac{|h|}{\sqrt{t}}\right)^{\tilde{v}} e^{\varepsilon t}
$$

for all $y \in \mathbb{R}^{d} \backslash N_{x, h, t, m}$. Then

$$
N=\bigcup_{x, h \in \mathbb{Q}^{d}} \bigcup_{t \in(0, \infty) \cap \mathbb{Q}} \bigcup_{m=1}^{d} N_{x, h, t, m}
$$

is a null set in $\mathbb{R}^{d}$ and (3.10) is valid for all $y \in \mathbb{R}^{d} \backslash N, x, h \in \mathbb{Q}^{d}, t \in(0, \infty) \cap \mathbb{Q}$ and $m \in\{1, \ldots, d\}$. Then by density and continuity (Statements (b) and (c)) one deduces that (3.10) is valid for all $y \in \mathbb{R}^{d} \backslash N, x, h \in \mathbb{R}^{d}, t>0$ and $m \in\{1, \ldots, d\}$.

Let $a, b$ be as in (3.8). Then it follows as in the proof of Step A of Lemma A.1 that there are $a_{2}, b_{2}>0$, depending only on $a, b, a_{1}, \kappa, \tau, \varepsilon, v$ and $\tilde{v}$, such that

$$
\begin{aligned}
& \left|\left(\partial_{x, m} K_{t}\right)(x, y)-\left(\partial_{x, m} K_{t}\right)(x-h, y)\right| \\
& \leq a_{2} t^{-d / 2} t^{-|\alpha| / 2}\left(\frac{|h|}{|x-y|+\sqrt{t}}\right)^{v} e^{\varepsilon t} e^{-b_{2} \frac{|x-y|^{2}}{t}}
\end{aligned}
$$

for all $y \in \mathbb{R}^{d} \backslash N, x, h \in \mathbb{R}^{d}, t>0$ and $m \in\{1, \ldots, d\}$ with $|h| \leq \tau|x-y|+$ $\kappa \sqrt{t}$. Now the theorem follows by replacing $(t, x, y) \mapsto K_{t}(x, y)$ by the function $(t, x, y) \mapsto K_{t}(x, y) \mathbb{1}_{\mathbb{R}^{d} \backslash N}(y)$.

Corollary 3.10. Let $\Omega \varsubsetneqq \mathbb{R}^{d}$ be open, $\theta \in\left[0, \frac{\pi}{2}\right), \mu, M>0$ and $\chi \in C_{\mathrm{b}}^{\infty}\left(\mathbb{R}^{d}\right)$ with $\chi \neq 0$ and $d\left(\operatorname{supp} \chi, \Omega^{\mathrm{c}}\right)>0$. Then there exists a $c>0$ such that $M_{\chi}(I+$ $A)^{-1} L_{p}\left(\mathbb{R}^{d}\right) \subset W^{1, p}\left(\mathbb{R}^{d}\right)$ and

$$
\left\|\partial_{m} M_{\chi} u\right\|_{p} \leq c\|(I+A) u\|_{p}
$$

for all $C \in \mathcal{S}^{1}(\Omega, \theta, \mu, M$, real $), p \in[1, \infty], u \in D(A)$ and $m \in\{1, \ldots, d\}$.

Proof. The Gaussian bounds of Theorem 3.9 imply bounds $\left\|\partial_{m} M_{\chi} S_{t}\right\|_{p \rightarrow p} \leq$ $c t^{-1 / 2} e^{t / 2}$. Then the corollary follows by a Laplace transform.

\section{Riesz transforms}

In this section we shall prove that various Riesz transforms like $\partial_{k} M_{\chi}(I+A)^{-1 / 2}$ and $\partial_{k} M_{\chi}(I+A)^{-1 / 2} M_{\chi}$ are bounded on $L_{2}$ or $L_{p}$. The first results on $L_{2}$ for the Riesz transform merely use that $\chi \in W^{1, \infty}\left(\mathbb{R}^{d}\right)$.

If $\eta \in W^{1, \infty}\left(\mathbb{R}^{d}\right)$, then

$$
\operatorname{Re} \mathfrak{a}_{C}(\eta u) \leq 2\|\eta\|_{\infty} \operatorname{Re} \mathfrak{a}_{C}(u)+2 M\|\nabla \eta\|_{\infty}^{2}\|u\|_{2}^{2}
$$


for all $u \in W^{1,2}\left(\mathbb{R}^{d}\right), \theta \in\left[0, \frac{\pi}{2}\right), M>0$ and $C \in \mathcal{S}(\theta, M)$. Therefore for self-adjoint operators the boundedness of the Riesz transforms on $L_{2}\left(\mathbb{R}^{d}\right)$ is trivial. Throughout this section let $L=-\sum_{k=1}^{d} \partial_{k}^{2}$ be the Laplacian and let $H=A_{\Re C}$.

The first lemma is a variation of Lemma 2.1(c).

Lemma 4.1. Let $\theta \in\left[0, \frac{\pi}{2}\right), \mu, M>0, \chi \in W^{1, \infty}\left(\mathbb{R}^{d}\right)$ and $C \in \mathcal{S}(\operatorname{supp} \chi, \theta, \mu, M)$. Then $M_{\chi} D\left(H^{1 / 2}\right) \subset W^{1,2}\left(\mathbb{R}^{d}\right)$ and

$$
\left\|\partial_{k} M_{\chi} u\right\|_{2} \leq\left\|(I+L)^{1 / 2} M_{\chi} u\right\|_{2} \leq c_{1}\left\|(I+H)^{1 / 2} u\right\|_{2}
$$

for all $u \in D\left(H^{1 / 2}\right)$ and $k \in\{1, \ldots, d\}$, where

$$
c_{1}=\left(\|\chi\|_{\infty}^{2}+2 \frac{\|\chi\|_{\infty}^{2}}{\mu}+2\|\nabla \chi\|_{\infty}^{2}\right)^{1 / 2} .
$$

Proof. We only have to prove the last estimate. It follows from Lemma 2.1(c) that

$$
\begin{aligned}
\left\|(I+L)^{1 / 2} M_{\chi} u\right\|_{2}^{2} & =\left\|M_{\chi} u\right\|_{2}^{2}+\sum_{k=1}^{d}\left\|\partial_{k} M_{\chi} u\right\|_{2}^{2} \\
& \leq\|\chi\|_{\infty}^{2}\|u\|_{2}^{2}+2 \sum_{k=1}^{d}\left\|\left(\partial_{k} M_{\chi}-M_{\partial_{k} \chi}\right) u\right\|_{2}^{2}+2 \sum_{k=1}^{d}\left\|M_{\partial_{k} \chi} u\right\|_{2}^{2} \\
& \leq\|\chi\|_{\infty}^{2}\|u\|_{2}^{2}+2 \frac{\|\chi\|_{\infty}^{2}}{\mu}\left\|H^{1 / 2} u\right\|_{2}^{2}+2\|(\nabla \chi) u\|_{2}^{2} \\
& \leq c_{1}^{2}\left\|(I+H)^{1 / 2} u\right\|_{2}^{2}
\end{aligned}
$$

as required.

Lemma 4.2. Let $\theta \in\left[0, \frac{\pi}{2}\right), \mu, M>0, \gamma \in(0,1)$ and $\chi \in W^{1, \infty}\left(\mathbb{R}^{d}\right)$. Then there exists a $c_{2}>0$ such that $M_{\chi} D\left(A^{\gamma / 2}\right) \subset D\left(L^{\gamma / 2}\right)$ and

$$
\left\|(I+L)^{\gamma / 2} M_{\chi} u\right\|_{2} \leq c_{2}\left\|(I+A)^{\gamma / 2} u\right\|_{2}
$$

for all $C \in \mathcal{S}(\operatorname{supp} \chi, \theta, \mu, M)$ and $u \in D\left((I+A)^{\gamma / 2}\right)$.

Proof. Let $c_{1}$ be as in Lemma 4.1. Then $M_{\chi}$ is continuous from $D\left((I+H)^{1 / 2}\right)$ into $D\left((I+L)^{1 / 2}\right.$ ) with norm bounded by $c_{1}$. Then by interpolation, Proposition $\mathrm{G}$ together with Theorem $\mathrm{G}$ in [1], it follows that $M_{\chi} D\left((I+H)^{\gamma / 2}\right) \subset D\left((I+L)^{\gamma / 2}\right)$ and

$$
\left\|(I+L)^{\gamma / 2} M_{\chi} u\right\|_{2} \leq c_{1}^{\prime}\left\|(I+H)^{\gamma / 2} u\right\|_{2}
$$

for all $u \in D\left((I+L)^{\gamma / 2}\right)$, where $c_{1}^{\prime}=c_{1}^{\gamma}\|\chi\|_{\infty}^{1-\gamma}$. But $D\left((I+H)^{\gamma / 2}\right)=D((I+$ $A)^{\gamma / 2}$ ) with equivalent norms by [13] Theorem 3.1. Explicitly,

$$
\left\|(I+H)^{\gamma / 2} u\right\|_{2} \leq \frac{1}{1-\tan \frac{\pi \gamma}{4}}\left\|(I+A)^{\gamma / 2} u\right\|_{2}
$$

for all $u \in D\left((I+A)^{\gamma / 2}\right)$. Then the lemma follows. 
Lemma 4.3. Let $\theta \in\left[0, \frac{\pi}{2}\right), \mu, M>0, \chi \in W^{1, \infty}\left(\mathbb{R}^{d}\right)$ and $C \in \mathcal{E}(\operatorname{supp} \chi, \theta, \mu, M)$. Then

$$
\left\|(I+H)^{1 / 2} M_{\chi} u\right\|_{2} \leq 2\left\|(I+H)^{-1 / 2} M_{\chi}(I+A) u\right\|_{2}+4 M^{1 / 2}\|\nabla \chi\|_{\infty}\|u\|_{2}
$$

for all $u \in D(A)$.

Proof. The proof is a variation of the proof of Lemma 1 in [14]. If $u \in D(A)$ then

$$
\begin{aligned}
\left\|(I+H)^{1 / 2} M_{\chi} u\right\|_{2}^{2}= & \operatorname{Re} \mathfrak{a}\left(M_{\chi} u\right)+\left\|M_{\chi} u\right\|_{2}^{2} \\
= & \operatorname{Re} \mathfrak{a}\left(u, M_{\chi}^{2} u\right)-\operatorname{Re} \sum\left(c_{k l} \partial_{k}(\chi u),\left(\partial_{l} \chi\right) u\right) \\
& +\operatorname{Re} \sum\left(c_{k l}\left(\partial_{k} \chi\right) u,\left(\partial_{l} \chi\right) u\right) \\
& +\operatorname{Re} \sum\left(c_{k l}\left(\partial_{k} \chi\right) u, \partial_{l}(\chi u)\right)+\left\|M_{\chi} u\right\|_{2}^{2} .
\end{aligned}
$$

But

$$
\begin{aligned}
\operatorname{Re} \mathfrak{a}\left(u, M_{\chi}^{2} u\right)+\left\|M_{\chi} u\right\|_{2}^{2} & =\operatorname{Re}\left(A u, M_{\chi}^{2} u\right)+\left\|M_{\chi} u\right\|_{2}^{2} \\
& =\operatorname{Re}\left((I+H)^{-1 / 2} M_{\chi}(I+A) u,(I+H)^{1 / 2} M_{\chi} u\right) \\
& \leq\left\|(I+H)^{-1 / 2} M_{\chi}(I+A) u\right\|_{2}\left\|(I+H)^{1 / 2} M_{\chi} u\right\|_{2} \\
& \leq \frac{1}{4}\left\|(I+H)^{1 / 2} M_{\chi} u\right\|_{2}^{2}+\left\|(I+H)^{-1 / 2} M_{\chi}(I+A) u\right\|_{2}^{2}
\end{aligned}
$$

and

$$
\begin{aligned}
- & \operatorname{Re} \sum\left(c_{k l} \partial_{k}(\chi u),\left(\partial_{l} \chi\right) u\right)+\operatorname{Re} \sum\left(c_{k l}\left(\partial_{k} \chi\right) u,\left(\partial_{l} \chi\right) u\right) \\
& +\operatorname{Re} \sum\left(c_{k l}\left(\partial_{k} \chi\right) u, \partial_{l}(\chi u)\right) \\
\leq & 2\left(\operatorname{Re} \mathfrak{a}\left(M_{\chi} u\right)\right)^{1 / 2}\left(\operatorname{Re} \sum \int c_{k l}\left(\partial_{k} \chi\right)\left(\partial_{l} \chi\right)|u|^{2}\right)^{1 / 2} \\
& +\operatorname{Re} \sum \int c_{k l}\left(\partial_{k} \chi\right)\left(\partial_{l} \chi\right)|u|^{2} \\
\leq & 2\left(\operatorname{Re} \mathfrak{a}\left(M_{\chi} u\right)\right)^{1 / 2} M^{1 / 2}\|\nabla \chi\|_{\infty}\|u\|_{2}+M\|\nabla \chi\|_{\infty}^{2}\|u\|_{2}^{2} \\
\leq & \frac{1}{4}\left\|(I+H)^{1 / 2} M_{\chi} u\right\|_{2}^{2}+5 M\|\nabla \chi\|_{\infty}^{2}\|u\|_{2}^{2} .
\end{aligned}
$$

So

$$
\left\|(I+H)^{1 / 2} M_{\chi} u\right\|_{2}^{2} \leq 2\left\|(I+H)^{-1 / 2} M_{\chi}(I+A) u\right\|_{2}^{2}+10 M\|\nabla \chi\|_{\infty}^{2}\|u\|_{2}^{2}
$$

and the lemma follows.

The next lemma is well known, but we need uniform constants. 
Lemma 4.4. Let $0<\gamma<v<1$ and $\eta \in C^{0, v}\left(\mathbb{R}^{d}\right) \cap L_{\infty}\left(\mathbb{R}^{d}\right)$. Let $u \in D\left(L^{\gamma / 2}\right)$. Then $M_{\eta} u \in D\left(L^{\gamma / 2}\right)$ and

$$
\left\|(I+L)^{\gamma / 2} M_{\eta} u\right\|_{2} \leq c_{4}\left(\|\eta\|_{\infty} \vee\|\eta \eta\|_{C^{0, v}}\right)\left\|(I+L)^{\gamma / 2} u\right\|_{2},
$$

where $c_{4}=1+c_{\gamma}^{-1} 2^{d+3} \Gamma\left(\frac{\nu-\gamma}{2}\right)$ and $c_{\gamma}=\int_{0}^{\infty} t^{-1-\frac{\gamma}{2}}\left(1-e^{-t}\right) d t$.

Proof. Let $T$ be the semigroup generated by $-L$ and for all $t>0$ let $G_{t}$ be the kernel of $T_{t}$. If $u \in L_{2}\left(\mathbb{R}^{d}\right)$ and $t>0$ then

$$
\left(\left[T_{t}, M_{\eta}\right] u\right)(x)=\int G_{t}(y)(\eta(x-y)-\eta(x)) u(x-y) d y
$$

for all $x \in \mathbb{R}^{d}$. But

$$
\left|G_{t}(y)(\eta(x-y)-\eta(x))\right| \leq 2 M G_{t}(y)|y|^{\nu} \leq 2^{d+3} M t^{\nu / 2} G_{2 t}(y)
$$

for all $x, y \in \mathbb{R}^{d}$, where $M=\|\eta\|_{\infty} \vee\|\| \eta \|_{C^{0, v}}$. Therefore $\left\|\left[T_{t}, M_{\eta}\right] u\right\|_{2} \leq$ $2^{d+3} M t^{\nu / 2}\|u\|_{2}$. Next,

$$
(I+L)^{\gamma / 2}=\frac{1}{c_{\gamma}} \int_{0}^{\infty} t^{-1-\frac{\gamma}{2}}\left(I-e^{-t} T_{t}\right) d t .
$$

So for all $u \in C_{c}^{\infty}\left(\mathbb{R}^{d}\right)$ one obtains

$$
\left[(I+L)^{\gamma / 2}, M_{\eta}\right] u=-\frac{1}{c_{\gamma}} \int_{0}^{\infty} t^{-1-\frac{\gamma}{2}} e^{-t}\left[T_{t}, M_{\eta}\right] u d t
$$

Therefore

$\left\|\left[(I+L)^{\gamma / 2}, M_{\eta}\right] u\right\|_{2} \leq \frac{2^{d+3} M}{c_{\gamma}} \int_{0}^{\infty} t^{-1+\frac{\nu-\gamma}{2}} e^{-t} d t\|u\|_{2}=\frac{2^{d+3} M \Gamma\left(\frac{\nu-\gamma}{2}\right)}{c_{\gamma}}\|u\|_{2}$

and

$$
\left\|(I+L)^{\gamma / 2} M_{\eta} u\right\|_{2} \leq c_{4}\left\|(I+L)^{\gamma / 2} u\right\|_{2} .
$$

Then by density the lemma follows.

Lemma 4.5. Let $\theta \in\left[0, \frac{\pi}{2}\right), \mu, M>0, v \in(0,1)$ and $\chi \in W^{1, \infty}\left(\mathbb{R}^{d}\right)$. Set $\gamma=\frac{v}{2}$.

Then there exists a $c_{5}>0$ such that $M_{\chi}^{2} D\left(L^{(1+\gamma) / 2}\right) \subset D\left(A^{(1+\gamma) / 2}\right)$ and

$$
\left\|(I+A)^{(1+\gamma) / 2} M_{\chi}^{2} u\right\|_{2} \leq c_{5}\left\|(I+L)^{(1+\gamma) / 2} u\right\|_{2}
$$

for all $C \in \mathcal{E}^{v}(\operatorname{supp} \chi, \theta, \mu, M)$ and $u \in D\left(L^{(1+\gamma) / 2}\right)$. 
Proof. The proof is a variation of the proof in [7]. Let $u \in D\left(L^{(1+\gamma) / 2}\right) \subset$ $W^{1,2}\left(\mathbb{R}^{d}\right)$ and $v \in D\left(A^{*}\right) \subset W^{1,2}\left(\mathbb{R}^{d}\right)$. Then

$$
\begin{aligned}
& \left(M_{\chi}^{2} u,\left(I+A^{*}\right)^{(1+\gamma) / 2} v\right) \\
& =\left(M_{\chi}^{2} u,\left(I+A^{*}\right)\left(I+A^{*}\right)^{-(1-\gamma) / 2} v\right) \\
& =\sum\left(c_{k l} \partial_{k} M_{\chi}^{2} u, \partial_{l}\left(I+A^{*}\right)^{-(1-\gamma) / 2} v\right)+\left(M_{\chi}^{2} u,\left(I+A^{*}\right)^{-(1-\gamma) / 2} v\right) \\
& =\sum\left(c_{k l} M_{\chi} \partial_{k} u, \partial_{l} M_{\chi}\left(I+A^{*}\right)^{-(1-\gamma) / 2} v\right) \\
& -\sum\left(c_{k l} M_{\chi} \partial_{k} u,\left(\partial_{l} \chi\right)\left(I+A^{*}\right)^{-(1-\gamma) / 2} v\right) \\
& +2 \sum\left(c_{k l}\left(\partial_{k} \chi\right) u, \partial_{l} M_{\chi}\left(I+A^{*}\right)^{-(1-\gamma) / 2} v\right) \\
& -2 \sum\left(c_{k l}\left(\partial_{k} \chi\right) u,\left(\partial_{l} \chi\right)\left(I+A^{*}\right)^{-(1-\gamma) / 2} v\right) \\
& +\left(M_{\chi} u, M_{\chi}\left(I+A^{*}\right)^{-(1-\gamma) / 2} v\right) \text {. }
\end{aligned}
$$

Fix $k, l \in\{1, \ldots, d\}$. Then $\chi c_{k l} \in C^{0, v}\left(\mathbb{R}^{d}\right) \cap L_{\infty}\left(\mathbb{R}^{d}\right)$ and \|\|$\chi c_{k l} \|_{C^{0, v}\left(\mathbb{R}^{d}\right)} \leq$ $2 M\|\chi\|_{W^{1, \infty}}$. So

$$
\begin{aligned}
& \left|\left(c_{k l} M_{\chi} \partial_{k} u, \partial_{l} M_{\chi}\left(I+A^{*}\right)^{-(1-\gamma) / 2} v\right)\right| \\
& \quad=\left|\left((I+L)^{\gamma / 2} M_{\chi c_{k l}} \partial_{k} u,(I+L)^{-\gamma / 2} \partial_{l} M_{\chi}\left(I+A^{*}\right)^{-(1-\gamma) / 2} v\right)\right| \\
& \quad \leq\left\|(I+L)^{\gamma / 2} M_{\chi c_{k l}} \partial_{k} u\right\|_{2}\left\|(I+L)^{-\gamma / 2} \partial_{l} M_{\chi}\left(I+A^{*}\right)^{-(1-\gamma) / 2} v\right\|_{2} .
\end{aligned}
$$

If $c_{4}$ is as in Lemma 4.4 , then

$$
\begin{aligned}
\left\|(I+L)^{\gamma / 2} M_{\chi c_{k l}} \partial_{k} u\right\|_{2} & \leq 2 c_{4} M\|\chi\|_{W^{1, \infty}}\left\|(I+L)^{\gamma / 2} \partial_{k} u\right\|_{2} \\
& \leq 2 c_{4} M\|\chi\|_{W^{1, \infty}}\left\|(I+L)^{(1+\gamma) / 2} u\right\|_{2} .
\end{aligned}
$$

Alternatively,

$$
\left\|(I+L)^{-\gamma / 2} \partial_{l} M_{\chi}\left(I+A^{*}\right)^{-(1-\gamma) / 2} v\right\|_{2} \leq\left\|(I+L)^{(1-\gamma) / 2} M_{\chi}\left(I+A^{*}\right)^{-(1-\gamma) / 2} v\right\|_{2} .
$$

By Lemma 4.2 there exists a $c_{2}>0$, depending only on $\theta, \mu, M, v$ and $\chi$, such that

$$
\left\|(I+L)^{(1-\gamma) / 2} M_{\chi} w\right\|_{2} \leq c_{2}\left\|\left(I+A^{*}\right)^{(1-\gamma) / 2} w\right\|_{2}
$$

for all $w \in D\left((I+L)^{(1-\gamma) / 2}\right)$. Hence

$$
\begin{aligned}
\left\|(I+L)^{-\gamma / 2} \partial_{l} M_{\chi}\left(I+A^{*}\right)^{-(1-\gamma) / 2} v\right\|_{2} & \leq c_{2}\left\|\left(I+A^{*}\right)^{(1-\gamma) / 2}\left(I+A^{*}\right)^{-(1-\gamma) / 2} v\right\|_{2} \\
& =c_{2}\|v\|_{2} .
\end{aligned}
$$

The other four terms in (4.1) can be estimated similarly.

Combining the contributions, it follows that there exists a $c>0$, depending only on $\theta, \mu, M, v$ and $\chi$, such that

$$
\left|\left(M_{\chi}^{2} u,\left(I+A^{*}\right)^{(1+\gamma) / 2} v\right)\right| \leq c\left\|(I+L)^{(1+\gamma) / 2} u\right\|_{2}\|v\|_{2}
$$


for all $v \in D\left(A^{*}\right)$. Since $D\left(A^{*}\right)$ is a core for $\left(I+A^{*}\right)^{(1+\gamma) / 2}$, one deduces that $M_{\chi}^{2} u \in D\left(A^{(1+\gamma) / 2}\right)$ and

$$
\left\|(I+A)^{(1+\gamma) / 2} M_{\chi}^{2} u\right\|_{2} \leq c\left\|(I+L)^{(1+\gamma) / 2} u\right\|_{2}
$$

as required.

Now we are able to prove a uniform version of Theorem 1.3(c).

Theorem 4.6. Let $\theta \in\left[0, \frac{\pi}{2}\right), \mu, M>0, v \in(0,1)$ and $\chi \in W^{1, \infty}\left(\mathbb{R}^{d}\right)$. Then there exists a $c>0$ such that $M_{\chi}^{3} u \in W^{1,2}\left(\mathbb{R}^{d}\right)$ and

$$
\left\|\partial_{k} M_{\chi}^{3} u\right\|_{2} \leq c\left\|(I+A)^{1 / 2} u\right\|_{2}
$$

for all $C \in \mathcal{S}^{v}(\operatorname{supp} \chi, \theta, \mu, M), u \in D\left(A^{1 / 2}\right)$ and $k \in\{1, \ldots, d\}$.

Proof. First suppose that $C \in \mathcal{E}^{v}(\Omega, \theta, \mu, M)$. Let $c_{5}>0$ be as in Lemma 4.5. Then by interpolation, Proposition $\mathrm{G}$ together with Theorem $\mathrm{G}$ in [1], one establishes that $M_{\chi}^{2} D\left((I+L)^{1 / 2}\right) \subset D\left((I+A)^{1 / 2}\right)$ and

$$
\left\|(I+A)^{1 / 2} M_{\chi}^{2} u\right\|_{2} \leq c_{5}^{\prime}\left\|(I+L)^{1 / 2} u\right\|_{2}
$$

for all $u \in W^{1,2}\left(\mathbb{R}^{d}\right)=D\left(L^{1 / 2}\right)$, where $c_{5}^{\prime}=c_{5}^{\frac{1}{1+\gamma}}\|\chi\|_{\infty}^{\frac{\gamma}{1+\gamma}}$. Hence if $c_{1}>0$ is as in Lemma 4.1 then

$$
\left\|(I+A)^{1 / 2} M_{\chi}^{3} u\right\|_{2} \leq c_{5}^{\prime}\left\|(I+L)^{1 / 2} M_{\chi} u\right\|_{2} \leq c_{1} c_{5}^{\prime}\left\|(I+H)^{1 / 2} u\right\|_{2} .
$$

So

$$
\left\|(I+A)^{1 / 2} M_{\chi}^{3}(I+H)^{-1 / 2}\right\|_{2 \rightarrow 2} \leq c_{1} c_{5}^{\prime}
$$

and then by duality

$$
\left\|(I+H)^{-1 / 2} M_{\chi}^{3}\left(I+A^{*}\right)^{1 / 2}\right\|_{2 \rightarrow 2} \leq c_{1} c_{5}^{\prime} .
$$

Since $\mathcal{E}^{v}(\Omega, \theta, \mu, M)$ is invariant under taking adjoints, one may replace $A^{*}$ by $A$ in (4.2). Then Lemma 4.3 gives

$$
\begin{aligned}
\left\|(I+H)^{1 / 2} M_{\chi}^{3} u\right\|_{2} & \leq 2\left\|(I+H)^{-1 / 2} M_{\chi}^{3}(I+A) u\right\|_{2}+c_{6}\|u\|_{2} \\
& \leq 2 c_{1} c_{5}^{\prime}\left\|(I+A)^{1 / 2} u\right\|_{2}+c_{6}\|u\|_{2} \\
& \leq\left(2 c_{1} c_{5}^{\prime}+c_{6}\right)\left\|(I+A)^{1 / 2} u\right\|_{2}
\end{aligned}
$$

for all $u \in D(A)$, where $c_{6}=4 M\left\|\nabla\left(\chi^{3}\right)\right\|_{\infty}$. Therefore $\left\|\partial_{k} M_{\chi}^{3} u\right\|_{2} \leq \mu^{-1 / 2}\left\|(I+H)^{1 / 2} M_{\chi}^{3} u\right\|_{2} \leq \mu^{-1 / 2}\left(2 c_{1} c_{5}^{\prime}+c_{6}\right)\left\|(I+A)^{1 / 2} u\right\|_{2}$. 
This extends to all $u \in D\left(A^{1 / 2}\right)$ by density. It follows that

$$
\left|\left(M_{\chi}^{3}(I+A)^{-1 / 2} u, \partial_{k} v\right)\right| \leq \mu^{-1 / 2}\left(2 c_{1} c_{5}^{\prime}+c_{6}\right)\|u\|_{2}\|v\|_{2}
$$

for all $u \in L_{2}\left(\mathbb{R}^{d}\right), v \in W^{1,2}\left(\mathbb{R}^{d}\right), k \in\{1, \ldots, d\}$ and $C \in \mathcal{E}^{v}(\operatorname{supp} \chi, \theta, \mu, M)$. By approximating $C$ by $C+\frac{1}{n} I$ it follows as before that (4.3) extends to all $C \in$ $\mathcal{S}^{v}(\operatorname{supp} \chi, \theta, \mu, M)$ and the theorem follows.

The theorem has many corollaries.

Corollary 4.7. Let $\Omega \varsubsetneqq \mathbb{R}^{d}$ be open, $\theta \in\left[0, \frac{\pi}{2}\right), \mu, M>0, v \in(0,1), p \in(1, \infty)$ and $\chi \in C_{\mathrm{b}}^{\infty}\left(\mathbb{R}^{d}\right)$ with $\chi \neq 0$ and $d\left(\operatorname{supp} \chi, \Omega^{\mathrm{c}}\right)>0$. Then there exists a $c>0$ such that

$$
\left\|\nabla M_{\chi}(I+A)^{-1 / 2} M_{\chi}\right\|_{p \rightarrow p} \leq c
$$

for all $C \in \mathcal{S}^{v}(\Omega, \theta, \mu, M)$.

Proof. For all $t>0$ let $K_{t}$ be the continuous kernel of the operator $M_{\chi} S_{t} M_{\chi}$. Fix $m \in\{1, \ldots, d\}$. By Theorem 2.3 there are $a>0$ and $b \in(0,1)$ such that

$$
\left|\left(\partial_{x, m} K_{t}\right)(x, y)\right| \leq a t^{-d / 2} t^{-1 / 2} e^{t / 2} e^{-b \frac{|x-y|^{2}}{t}}
$$

and

$$
\left|\left(\partial_{x, m} K_{t}\right)(x+h, y+k)-\left(\partial_{x, m} K_{t}\right)(x, y)\right| \leq a t^{-d / 2} t^{-|\alpha| / 2}\left(\frac{|h|+|k|}{\sqrt{t}}\right)^{v} e^{t} e^{-b \frac{|x-y|^{2}}{t}}
$$

for all $t>0$ and $x, y, h, k \in \mathbb{R}^{d}$ with $|h|+|k| \leq \frac{1}{2}|x-y|$. For all $x, y \in \mathbb{R}^{d}$ with $x \neq y$ define

$$
L(x, y)=\frac{1}{\sqrt{\pi}} \int_{0}^{\infty} t^{-1 / 2} e^{-t}\left(\partial_{x, m} K_{t}\right)(x, y) d t .
$$

Then

$$
\left(\partial_{m} M_{\chi}(I+A)^{-1 / 2} M_{\chi} u\right)(x)=\int_{\mathbb{R}^{d}} L(x, y) u(y) d y
$$

for all $u \in L_{2}\left(\mathbb{R}^{d}\right)$ and $x \in \mathbb{R}^{d} \backslash \operatorname{supp} u$. Moreover, for all $x, y, h \in \mathbb{R}^{d}$ with $|h| \leq \frac{1}{2}|x-y|$ one has

$$
\begin{aligned}
|L(x+h, y)-L(x, y)| & \leq \frac{a}{\sqrt{\pi}} \int_{0}^{\infty} t^{-d / 2} t^{-1}\left(\frac{|h|}{\sqrt{t}}\right)^{v} e^{-b \frac{|x-y|^{2}}{t}} d t \\
& =c\left(\frac{|h|}{|x-y|}\right)^{v} \frac{1}{|x-y|^{d}}
\end{aligned}
$$


where $c=\frac{a}{\sqrt{\pi}} \int_{0}^{\infty} t^{-1-\frac{d+v}{2}} e^{-\frac{b}{t}} d t$. Similarly,

$$
|L(x, y+k)-L(x, y)| \leq c\left(\frac{|h|}{|x-y|}\right)^{v} \frac{1}{|x-y|^{d}}
$$

for all $x, y, k \in \mathbb{R}^{d}$ with $|k| \leq \frac{1}{2}|x-y|$. In addition,

$$
|L(x, y)| \leq \frac{a}{\sqrt{\pi}} \frac{1}{|x-y|^{d}} \int_{0}^{\infty} t^{-1-\frac{d+v}{2}} e^{-\frac{b}{t}} d t
$$

for all $x, y \in \mathbb{R}^{d}$ with $x \neq y$. Therefore $\partial_{m} M_{\chi}(I+A)^{-1 / 2} M_{\chi}$ is a CalderónZygmund operator. Since it is bounded on $L_{2}\left(\mathbb{R}^{d}\right)$ by Theorem 4.6 , it is also bounded in $L_{p}\left(\mathbb{R}^{d}\right)$ for all $p \in(1, \infty)$ by Theorem L in [1], or [20].

Corollary 4.8. Let $\Omega \varsubsetneqq \mathbb{R}^{d}$ be open, $\theta \in\left[0, \frac{\pi}{2}\right), \mu, M>0, v \in(0,1), p \in$ $(1, \infty), k, l \in\{1, \ldots, d\}$ and $\chi \in C_{\mathrm{b}}^{\infty}\left(\mathbb{R}^{d}\right)$ with $\chi \neq 0$ and $d\left(\operatorname{supp} \chi, \Omega^{\mathrm{c}}\right)>0$. Then there exists a $c>0$ such that

$$
\left\|\partial_{k} M_{\chi}(I+A)^{-1} M_{\chi} \partial_{l}\right\|_{p \rightarrow p} \leq c
$$

for all $C \in \mathcal{S}^{v}(\Omega, \theta, \mu, M)$.

Proof. Note that in $L_{2}$ the operator $\partial_{k} M_{\chi}(I+A)^{-1 / 2}$ is bounded by Theorem 4.6. So by duality the theorem follows for $p=2$. Then the rest of the proof is similar to the proof of Corollary 4.7.

Corollary 4.9. Let $\Omega \varsubsetneqq \mathbb{R}^{d}$ be open, $\theta \in\left[0, \frac{\pi}{2}\right), \mu, M>0, p \in(1, \infty), m \in$ $\{1, \ldots, d\}$ and $\chi \in C_{\mathrm{b}}^{\infty}\left(\mathbb{R}^{d}\right)$ with $\chi \neq 0$ and $d\left(\operatorname{supp} \chi, \Omega^{\mathrm{c}}\right)>0$. Then there exists ac $>0$ such that

$$
\left\|\partial_{m} M_{\chi}(I+A)^{-1 / 2}\right\|_{p \rightarrow p} \leq c
$$

for all $C \in \mathcal{S}^{1}(\Omega, \theta, \mu, M$, real $)$.

Proof. It suffices to prove the corollary with $M_{\chi}$ replaced by $M_{\chi}^{2}$. We use again a commutator. It follows from Lemma 3.8 that on $L_{2}\left(\mathbb{R}^{d}\right)$ one has

$$
\begin{aligned}
\partial_{m} & M_{\chi}^{2}(I+A)^{-1 / 2}-\partial_{m} M_{\chi}(I+A)^{-1 / 2} M_{\chi} \\
& =\frac{1}{\sqrt{\pi}} \int_{0}^{\infty} t^{-1 / 2} e^{-t} \partial_{m} M_{\chi}\left[M_{\chi}, S_{t}\right] d t \\
& =-\frac{1}{\sqrt{\pi}} \sum_{k, l=1}^{d} \int_{0}^{\infty} \int_{0}^{t} t^{-1 / 2} e^{-t} \partial_{m} M_{\chi} S_{s} M_{\partial_{k} \chi} \partial_{l} M_{\tilde{c}_{k l}} M_{\tilde{\chi}} S_{t-s} d s d t+R,
\end{aligned}
$$


where $R$ is the contribution of the last term in (3.7) and $\tilde{\chi} \in C_{\mathrm{b}}^{\infty}\left(\mathbb{R}^{d}\right)$ is such that $d\left(\operatorname{supp} \tilde{\chi}, \Omega^{\mathrm{c}}\right)>0$ and $\tilde{\chi}(x)=1$ for all $x \in \operatorname{supp} \chi$. Using the Gaussian bounds of Theorem 3.9 it follows that there exists a $c>0$ such that

$$
\begin{aligned}
\left\|\partial_{m} M_{\chi} S_{t} M_{\partial_{k} \chi}\right\|_{p \rightarrow p} & \leq c t^{-1 / 2} e^{t / 2} \text { and } \\
\left\|\partial_{l} M_{\tilde{c}_{k l}} M_{\tilde{\chi}} S_{t}\right\|_{p \rightarrow p} & \leq c t^{-1 / 2} e^{t / 2}
\end{aligned}
$$

for all $k, l \in\{1, \ldots, d\}$ and $t>0$. Then

$$
\begin{aligned}
& \left\|\int_{0}^{\infty} \int_{0}^{t} t^{-1 / 2} e^{-t} \partial_{m} M_{\chi} S_{s} M_{\partial_{k} \chi} \partial_{l} M_{\tilde{c}_{k l}} M_{\tilde{\chi}} S_{t-s} d s d t\right\|_{p \rightarrow p} \\
& \quad \leq c^{2} \int_{0}^{\infty} \int_{0}^{t} t^{-1 / 2} e^{-t / 2} s^{-1 / 2}(t-s)^{-1 / 2} d s d t \\
& \quad=c^{2} \pi \sqrt{2 \pi} .
\end{aligned}
$$

The contribution of $R$ can be estimated similarly and the current corollary follows from Corollary 4.7.

We end with two propositions on second-order Riesz transforms.

Proposition 4.10. Let $\Omega \varsubsetneqq \mathbb{R}^{d}$ be open, $\theta \in\left[0, \frac{\pi}{2}\right), \mu, M>0, p \in(1, \infty)$, $m, n \in\{1, \ldots, d\}$ and $\chi \in C_{\mathrm{b}}^{\infty}\left(\mathbb{R}^{d}\right)$ with $\chi \neq 0$ and $d\left(\operatorname{supp} \chi, \Omega^{\mathrm{c}}\right)>0$. Then there exists a $c>0$ such that $M_{\chi}(I+A)^{-1} L_{p}\left(\mathbb{R}^{d}\right) \subset W^{2, p}\left(\mathbb{R}^{d}\right)$ and

$$
\left\|\partial_{m} \partial_{n} M_{\chi}(I+A)^{-1}\right\|_{p \rightarrow p} \leq c
$$

for all $C \in \mathcal{S}^{1}(\Omega, \theta, \mu, M$, real $)$.

Proof. First let $C \in \mathcal{E} \mathcal{H}^{1}\left(\Omega, \theta, \mu, M\right.$, real). Let $F=\operatorname{supp} \chi$ and define $\chi_{1}, \chi_{2}$ as in (2.4) and (2.5). Let $M^{\prime}=2\left\|\chi_{2}\right\|_{W^{1, \infty}\left(\mathbb{R}^{d}\right)} M+\|\chi\|_{W^{1, \infty}\left(\mathbb{R}^{d}\right)}+1$ and $C^{\prime}=$ $\chi_{2} C+\chi_{1} I$. Then $C^{\prime} \in \mathcal{E} \mathcal{H}^{1}\left(\mathbb{R}^{d}, \theta, \mu \wedge 1, M^{\prime}\right)$. By [8] Proposition 5.1 there exists a suitable $c>0$ such that $\left(I+A^{\prime}\right)^{-1} L_{p}\left(\mathbb{R}^{d}\right) \subset W^{2, p}\left(\mathbb{R}^{d}\right)$ and

$$
\left\|\partial_{m} \partial_{n} u\right\|_{p \rightarrow p} \leq c\left\|\left(I+A^{\prime}\right) u\right\|_{p}
$$

for all $u \in W^{2, p}\left(\mathbb{R}^{d}\right)$, where $A^{\prime}=A_{C^{\prime}}$. Then

$$
\left\|\partial_{m} \partial_{n} M_{\chi} u\right\|_{p \rightarrow p} \leq c\left\|\left(I+A^{\prime}\right) M_{\chi} u\right\|_{p}=c\left\|(I+A) M_{\chi} u\right\|_{p} .
$$

But

$$
(I+A) M_{\chi} u=M_{\chi}(I+A) u-\sum_{k, l=1}^{d}\left(\left(\partial_{l} \chi\right) c_{k l} \partial_{k} u+\partial_{l} c_{k l}\left(\partial_{k} \chi\right) u\right) .
$$

Hence it follows from Corollary 3.10 that there exists a suitable $c^{\prime}>0$ such that

$$
\left\|\partial_{m} \partial_{n} M_{\chi} u\right\|_{p \rightarrow p} \leq c^{\prime}\|(I+A) u\|_{p}
$$

for all $u \in W^{2, p}\left(\mathbb{R}^{d}\right)=D(A)$. Then the proposition follows by approximation. 
On $L_{2}$ the same argument works for operators with complex coefficients.

Proposition 4.11. Let $\Omega \varsubsetneqq \mathbb{R}^{d}$ be open, $\theta \in\left[0, \frac{\pi}{2}\right), \mu, M>0, p \in(1, \infty)$, $m, n \in\{1, \ldots, d\}$ and $\chi \in C_{\mathrm{b}}^{\infty}\left(\mathbb{R}^{d}\right)$ with $\chi \neq 0$ and $d\left(\operatorname{supp} \chi, \Omega^{\mathrm{c}}\right)>0$. Then there exists a $c>0$ such that $M_{\chi}(I+A)^{-1} L_{2}\left(\mathbb{R}^{d}\right) \subset W^{2,2}\left(\mathbb{R}^{d}\right)$ and

$$
\left\|\partial_{m} \partial_{n} M_{\chi}(I+A)^{-1}\right\|_{2 \rightarrow 2} \leq c
$$

for all $C \in \mathcal{S}^{1}(\Omega, \theta, \mu, M)$.

\section{A. Gaussian bounds}

The main aim of this appendix is to transfer weighted semigroup bounds into Gaussian kernel bounds, with optimal large time behaviour and optimal control of the constant in the Gaussian, including the Hölder bounds. Throughout this appendix we write $C^{v}=C^{0, v}\left(\mathbb{R}^{d}\right)$ for all $v \in(0,1)$.

Lemma A.1. Let $N, N^{*} \in \mathbb{N}_{0}$ and $v, v^{*} \in(0,1)$. Let $S$ be a $C_{0}$-semigroup on $L_{2}\left(\mathbb{R}^{d}\right)$ and $T_{1}, T_{2} \in \mathcal{L}\left(L_{2}\left(\mathbb{R}^{d}\right)\right)$ such that $T_{1} S_{t}\left(C_{c}^{\infty}\left(\mathbb{R}^{d}\right)\right) \subset W^{N+v, \infty}\left(\mathbb{R}^{d}\right)$ and $T_{2}^{*} S_{t}^{*}\left(C_{c}^{\infty}\left(\mathbb{R}^{d}\right)\right) \subset W^{N^{*}+v^{*}, \infty}\left(\mathbb{R}^{d}\right)$ for all $t>0$. Assume that $\left[T_{1}, U_{\rho}\right]=$ $\left[T_{2}, U_{\rho}\right]=0$ for all $\rho \in \mathbb{R}$ and $\psi \in \mathcal{D}_{1}$. Let $a_{0}, a_{1}, \omega_{1}, \omega>0$ and suppose that

$$
\left\|U_{\rho} S_{t} U_{-\rho}\right\|_{2 \rightarrow 2} \leq a_{0} e^{\omega \rho^{2} t}
$$

for all $t>0, \rho \in \mathbb{R}$ and $\psi \in \mathcal{D}_{1}$. Moreover, suppose that

$$
\begin{aligned}
\left\|\partial^{\alpha} U_{\rho} T_{1} S_{t} U_{-\rho} u\right\|_{C^{\nu}} & \leq a_{1} t^{-d / 4} t^{-|\alpha| / 2} t^{-v / 2} e^{\omega_{1}\left(1+\rho^{2}\right) t}\|u\|_{2}, \\
\|\| \partial^{\beta} U_{\rho} T_{2}^{*} S_{t}^{*} U_{-\rho} u \|_{C^{\nu^{*}}} & \leq a_{1} t^{-d / 4} t^{-|\beta| / 2} t^{-v^{*} / 2} e^{\omega_{1}\left(1+\rho^{2}\right) t}\|u\|_{2}
\end{aligned}
$$

for all $t>0, \rho \in \mathbb{R}, \psi \in \mathcal{D}_{|\alpha| \vee|\beta| \vee 1}, u \in C_{c}^{\infty}\left(\mathbb{R}^{d}\right)$ and multi-indices $\alpha, \beta$ with $|\alpha| \leq N$ and $|\beta| \leq N^{*}$. Let $c>0$ be such that

$$
\sup \left\{\psi(x)-\psi(y): \psi \in \mathcal{D}_{N \vee N^{*} \vee 1}\right\} \geq c|x-y|
$$

for all $x, y \in \mathbb{R}^{d}$ and set $b=\frac{c^{2}}{4 \omega}$.

Then for all $t>0$ the operator $T_{1} S_{t} T_{2}$ has a continuous kernel $K_{t}$ which is $N_{1}$-times differentiable in the first variable and $N_{2}$-times in the second one, in any order. Moreover, there exists an a $>0$, depending only on $a_{0}, a_{1}, \omega, \omega_{1}, N, N^{*}, v$, $\nu^{*},\left\|T_{1}\right\|$ and $\left\|T_{2}\right\|$ such that

$$
\left|\left(\partial_{x}^{\alpha} \partial_{y}^{\beta} K_{t}\right)(x, y)\right| \leq a t^{-\frac{d+|\alpha|+|\beta|}{2}}\left(1+t+\frac{|x-y|^{2}}{t}\right)^{\frac{d+|\alpha|+|\beta|}{2}} e^{-b \frac{|x-y|^{2}}{t}}
$$

for all $x, y \in \mathbb{R}^{d}, t>0$ and multi-indices $\alpha, \beta$ with $|\alpha| \leq N$ and $|\beta| \leq N^{*}$. 
Finally, let $\gamma, \gamma^{*} \in(0,1), \kappa>0, \tau \in[0,1)$,

$$
0<b_{1}<b(1-\tau)^{2} \frac{c}{c+2 \tau}
$$

and $\alpha, \beta$ multi-indices with $|\alpha| \leq N,|\beta| \leq N^{*},|\alpha|+\gamma \leq N+v$ and $|\beta|+\gamma^{*} \leq$ $N^{*}+v^{*}$. Then there exists an $a>0$, depending only on $a_{0}, a_{1}, b_{1}, N, N^{*}, v, v^{*}$, $\gamma, \gamma^{*}, \kappa, \tau,\left\|T_{1}\right\|$ and $\left\|T_{2}\right\|$ such that

$$
\begin{aligned}
& \left|\left(\partial_{x}^{\alpha} \partial_{y}^{\beta} K_{t}\right)(x+h, y+k)-\left(\partial_{x}^{\alpha} \partial_{y}^{\beta} K_{t}\right)(x, y)\right| \\
& \quad \leq a t^{-\frac{d+|\alpha|+|\beta|}{2}}(1+t)^{\frac{d+|\alpha|+|\beta|+\gamma+\gamma^{*}}{2}}\left(\left(\frac{|h|}{\sqrt{t}+|x-y|}\right)^{\gamma}+\left(\frac{|k|}{\sqrt{t}+|x-y|}\right)^{\gamma^{*}}\right) e^{-b_{1} \frac{|x-y|^{2}}{t}}
\end{aligned}
$$

for all $x, y, h, k \in \mathbb{R}^{d}$ and $t>0$ with $|h|+|k| \leq \kappa \sqrt{t}+\tau|x-y|$.

In the proof of Lemma A.1 we need some estimates which are of independent interest.

Lemma A.2. Let $v \in(0,1)$.

(a) If $u \in C^{v} \cap L_{2}$ then $u \in L_{\infty}$ and

$$
\|u\|_{\infty} \leq \frac{d}{d+v} \varepsilon^{v}\left|\|u\|\left\|_{C^{v}}+|B(1)|^{-1 / 2} \varepsilon^{-d / 2}\right\| u \|_{2}\right.
$$

for all $\varepsilon \in(0,1]$.

(b) If $k \in\{1, \ldots, d\}$ and $u \in W^{1+v, \infty}\left(\mathbb{R}^{d}\right)$ then

$$
\left\|\partial_{k} u\right\|_{\infty} \leq \frac{1}{1+v} \varepsilon^{v}\left\|\partial_{k} u\right\| C_{C^{v}}+\varepsilon^{-(1-v)}\|\| u\|\|_{C^{v}}
$$

for all $\varepsilon \in(0,1]$.

(c) If $u \in W^{1, \infty}\left(\mathbb{R}^{d}\right)$, then

$$
\|\| u\|\|_{C^{\gamma}} \leq 2\|\nabla u\|_{\infty}^{\gamma}\|u\|_{\infty}^{1-\gamma}
$$

for all $\gamma \in(0,1)$.

(d) If $0<\gamma<v$, then

$$
\left.\|\| u\left\|\left.\right|_{C^{\gamma}} \leq 2\right\|\|u\|\right|_{C^{v}} ^{\frac{\gamma}{v}}\|u\|_{\infty}^{1-\frac{\gamma}{v}}
$$

for all $u \in C^{v} \cap L_{\infty}$.

Proof. Let $x \in \mathbb{R}^{d}$ and $h \in B(\varepsilon)$. Then $|u(x)| \leq|u(x)-u(x+h)|+|u(x+h)| \leq$ $|h|^{v}|||u| \|_{C^{\nu}}+|u(x+h)|$. Integration over $h$ gives

$$
\begin{aligned}
\varepsilon^{d}|B(1)||u(x)| & \leq|\|u\||_{C^{v}} \int_{B(\varepsilon)}|h|^{v} d h+\int_{B(\varepsilon)}|u(x+h)| d h \\
& \leq|\|u\||_{C^{v}} d|B(1)| \int_{0}^{\varepsilon} \rho^{v} \rho^{d-1} d \rho+|B(\varepsilon)|^{1 / 2}\|u\|_{2} \\
& =\left|\|u\|\left\|_{C^{v}} \frac{d}{d+v}|B(1)| \varepsilon^{d+v}+|B(1)|^{1 / 2} \varepsilon^{d / 2}\right\| u \|_{2}\right.
\end{aligned}
$$


from which Statement (a) follows.

For Statement (b) note that

$$
\begin{aligned}
u\left(x+\varepsilon e_{k}\right)-u(x) & =\int_{0}^{\varepsilon}\left(\partial_{k} u\right)\left(x+t e_{k}\right) d t \\
& =\varepsilon\left(\partial_{k} u\right)(x)+\int_{0}^{\varepsilon}\left(\left(\partial_{k} u\right)\left(x+t e_{k}\right)-\left(\partial_{k} u\right)(x)\right) d t
\end{aligned}
$$

So

$$
\begin{aligned}
\left|\left(\partial_{k} u\right)(x)\right| & \leq \frac{1}{\varepsilon}\left|u\left(x+\varepsilon e_{k}\right)-u(x)\right|+\frac{1}{\varepsilon} \int_{0}^{\varepsilon}\left|\left(\partial_{k} u\right)\left(x+t e_{k}\right)-\left(\partial_{k} u\right)(x)\right| d t \\
& \leq \varepsilon^{-(1-v)}|\|u\||_{C^{v}}+\left.\frac{1}{\varepsilon} \int_{0}^{\varepsilon} t^{v}\left\|\partial_{k} u\right\|\right|_{C^{v}} d t \\
& =\varepsilon^{-(1-v)} \mid\|u\|\left\|_{C^{v}}+\frac{1}{1+v} \varepsilon^{v}\right\| \partial_{k} u\|\|_{C^{v}}
\end{aligned}
$$

for all $x \in \mathbb{R}^{d}$.

The proof of Statements (c) and (d) is easy.

Proof of Lemma A.1. We follow arguments as in [9,10] and [18]. Set $N_{0}=N \vee$ $N^{*} \vee 1$.

Step 1. Since $T_{1}$ and $T_{2}$ commute with $U_{\rho}$ one has estimates

$$
\left\|U_{\rho} T_{1} S_{t} U_{-\rho}\right\|_{2 \rightarrow 2} \leq a_{0}\left\|T_{1}\right\| e^{\omega \rho^{2} t}
$$

and similarly for $T_{2}$. It then follows from the first two statements of Lemma A.2 with $\varepsilon=t^{1 / 2} e^{-t}$ that

$$
\begin{aligned}
\left\|\partial^{\alpha} U_{\rho} T_{1} S_{t} U_{-\rho} u\right\|_{\infty} & \leq \frac{1}{2} a_{2} t^{-d / 4} t^{-|\alpha| / 2} e^{\omega_{2}\left(1+\rho^{2}\right) t}\|u\|_{2}, \\
\left\|\partial^{\beta} U_{\rho} T_{2}^{*} S_{t}^{*} U_{-\rho} u\right\|_{\infty} & \leq \frac{1}{2} a_{2} t^{-d / 4} t^{-|\beta| / 2} e^{\omega_{2}\left(1+\rho^{2}\right) t}\|u\|_{2}
\end{aligned}
$$

for all $t>0, \rho \in \mathbb{R}, \psi \in \mathcal{D}_{N_{0}}, u \in C_{c}^{\infty}\left(\mathbb{R}^{d}\right)$ and multi-indices $\alpha, \beta$ with $|\alpha| \leq N$ and $|\beta| \leq N^{*}$, where $a_{2}=4 a_{1}+2|B(1)|^{-1 / 2}\left(\left\|T_{1}\right\| \vee\left\|T_{2}\right\|\right) a_{0}$ and $\omega_{2}=\left(\omega_{1}+\right.$ $1-\nu) \vee\left(\omega+\frac{d}{2}\right)$. Then using the last two statements of Lemma A.2 one deduces that

$$
\begin{aligned}
&\left\|\partial^{\alpha} U_{\rho} T_{1} S_{t} U_{-\rho} u\right\|_{C^{\gamma}} \leq a_{2} t^{-d / 4} t^{-|\alpha| / 2} t^{-\gamma / 2} e^{\omega_{2}\left(1+\rho^{2}\right) t}\|u\|_{2}, \\
&\|\| \partial^{\beta} U_{\rho} T_{2}^{*} S_{t}^{*} U_{-\rho} u\left\|_{C}\right\| \leq a_{2} t^{-d / 4} t^{-|\beta| / 2} t^{-\gamma^{*} / 2} e^{\omega_{2}\left(1+\rho^{2}\right) t}\|u\|_{2}
\end{aligned}
$$

for all $t>0, \rho \in \mathbb{R}, \psi \in \mathcal{D}_{N_{0}}, u \in C_{c}^{\infty}\left(\mathbb{R}^{d}\right)$, multi-indices $\alpha, \beta$ and $\gamma, \gamma^{*} \in(0,1)$ with $|\alpha|+\gamma \leq N+v$ and $|\beta|+\gamma^{*} \leq N^{*}+v^{*}$. 
Step 2. Let $\gamma \in(0,1)$ and $\alpha$ be a multi-index with $|\alpha|+\gamma \leq N+v$. Note that $U_{\rho} \partial_{k}=\partial_{k} U_{\rho}+\rho M_{\partial_{k} \psi} U_{\rho}$ and $|\rho| t^{(n+1) / 2} \leq n ! e^{\left(1+\rho^{2}\right) t}$ for all $n \in \mathbb{N}_{0}$. Hence it follows by induction to $\left|\alpha^{\prime \prime}\right|$ that

$$
\begin{aligned}
& \|\| \partial^{\alpha^{\prime}} U_{\rho} \partial^{\alpha^{\prime \prime}} T_{1} S_{t} U_{-\rho} u\|\|_{C \gamma} \\
& \quad \leq\left(1+N ! 2^{N}\right)^{\left|\alpha^{\prime \prime}\right|} a_{2} t^{-d / 4} t^{-\left(\left|\alpha^{\prime}\right|+\left|\alpha^{\prime \prime}\right|\right) / 2} t^{-\gamma / 2} e^{\left(\omega_{2}+\left|\alpha^{\prime \prime}\right|\right)\left(1+\rho^{2}\right) t}\|u\|_{2}
\end{aligned}
$$

for all $t>0, \rho \in \mathbb{R}, \psi \in \mathcal{D}_{N_{0}}, u \in L_{2}$ and multi-indices $\alpha^{\prime}, \alpha^{\prime \prime}$ with $\left|\alpha^{\prime}\right|+\left|\alpha^{\prime \prime}\right| \leq$ $|\alpha|$. In particular,

$$
\left\|\mid U_{\rho} \partial^{\alpha} T_{1} S_{t} U_{-\rho} u\right\|_{C^{\gamma}} \leq a_{2} c_{1} t^{-d / 4} t^{-|\alpha| / 2} t^{-\gamma / 2} e^{\left(\omega_{2}+N\right)\left(1+\rho^{2}\right) t}\|u\|_{2},
$$

where $c_{1}=\left(1+N ! 2^{N}\right)^{N}$. Similarly,

$$
\left\|U_{\rho} \partial^{\alpha} T_{1} S_{t} U_{-\rho} u\right\|_{\infty} \leq a_{2} c_{1} t^{-d / 4} t^{-|\alpha| / 2} e^{\left(\omega_{2}+N\right)\left(1+\rho^{2}\right) t}\|u\|_{2} .
$$

If $h \in \mathbb{R}^{d}$ with $|h| \geq 1$ then

$$
\begin{aligned}
\| & (I-L(h)) U_{\rho} \partial^{\alpha} T_{1} S_{t} U_{-\rho} u \|_{\infty} \\
\quad & \leq 2 a_{2} c_{1} t^{-d / 4} t^{-|\alpha| / 2} e^{\left(\omega_{2}+N\right)\left(1+\rho^{2}\right) t}\|u\|_{2} \\
& \leq 2 a_{2} c_{1} t^{-d / 4} t^{-|\alpha| / 2}\left(\frac{|h|}{\sqrt{t}}\right)^{\gamma} e^{\left(\omega_{2}+N+1\right)\left(1+\rho^{2}\right) t}\|u\|_{2} .
\end{aligned}
$$

So

$$
\left\|(I-L(h)) U_{\rho} \partial^{\alpha} T_{1} S_{t} U_{-\rho} u\right\|_{\infty} \leq a_{3} t^{-d / 4} t^{-|\alpha| / 2}\left(\frac{|h|}{\sqrt{t}}\right)^{\gamma} e^{\omega_{3}\left(1+\rho^{2}\right) t}\|u\|_{2}
$$

for all $t>0, h \in \mathbb{R}^{d}, \rho \in \mathbb{R}, \psi \in \mathcal{D}_{N_{0}}$ and $u \in L_{2}$, where $a_{3}=2 a_{2} c_{1}$ and $\omega_{3}=\omega_{2}+N+1$.

Step 3. Let $\gamma \in(0,1)$ and $\alpha$ be a multi-index with $|\alpha|+\gamma \leq N+v$. Let $h \in \mathbb{R}^{d}$, $\psi \in \mathcal{D}_{N_{0}}, \rho \in \mathbb{R}$ and $u \in L_{2}$. Set $t_{0}=\frac{1}{\omega_{3}\left(1+\rho^{2}\right)}$. If $t \in\left(0, t_{0}\right]$ then

$$
\left\|(I-L(h)) U_{\rho} \partial^{\alpha} T_{1} S_{t} U_{-\rho} u\right\|_{\infty} \leq a_{3} e t^{-d / 4} t^{-|\alpha| / 2}\left(\frac{|h|}{\sqrt{t}}\right)^{\gamma}\|u\|_{2}
$$

and if $t \in\left(t_{0}, \infty\right)$ then

$$
\begin{aligned}
& \left\|(I-L(h)) U_{\rho} \partial^{\alpha} T_{1} S_{t} U_{-\rho} u\right\|_{\infty} \\
& \quad=\left\|(I-L(h)) U_{\rho} \partial^{\alpha} T_{1} S_{t_{0}} U_{-\rho} U_{\rho} S_{t-t_{0}} U_{-\rho} u\right\|_{\infty} \\
& \quad \leq a_{3} e t_{0}^{-d / 4} t_{0}^{-|\alpha| / 2}\left(\frac{|h|}{\sqrt{t_{0}}}\right)^{\gamma}\left\|U_{\rho} S_{t-t_{0}} U_{-\rho} u\right\|_{2} \\
& \quad \leq a_{0} a_{3} e\left(\omega_{3}\left(1+\rho^{2}\right) t\right)^{\frac{d}{4}+\frac{|\alpha|+\gamma}{2}} t^{-d / 4} t^{-|\alpha| / 2}\left(\frac{|h|}{\sqrt{t}}\right)^{\gamma} e^{\omega \rho^{2}\left(t-t_{0}\right)}\|u\|_{2} .
\end{aligned}
$$


Hence

$$
\begin{aligned}
\|(I- & L(h)) U_{\rho} \partial^{\alpha} T_{1} S_{t} U_{-\rho} u \|_{\infty} \\
& \leq a_{4}\left(1+\omega_{3}\left(1+\rho^{2}\right) t\right)^{\frac{d}{4}+\frac{|\alpha|+\gamma}{2}} t^{-d / 4} t^{-|\alpha| / 2}\left(\frac{|h|}{\sqrt{t}}\right)^{\gamma} e^{\omega \rho^{2} t}\|u\|_{2}
\end{aligned}
$$

for all $t>0$, where $a_{4}=a_{3}\left(1 \vee a_{0}\right) e$. Similarly,

$$
\left\|U_{\rho} \partial^{\alpha} T_{1} S_{t} U_{-\rho} u\right\|_{\infty} \leq a_{4}\left(1+\omega_{3}\left(1+\rho^{2}\right) t\right)^{\frac{d}{4}+\frac{|\alpha|}{2}} t^{-d / 4} t^{-|\alpha| / 2} e^{\omega \rho^{2} t}\|u\|_{2}
$$

for all $\psi \in \mathcal{D}_{N_{0}}, \rho \in \mathbb{R}, t>0,|\alpha| \leq N$ and $u \in L_{2}$. Also similar bounds are valid with $T_{2}^{*}$ and $S_{t}^{*}$.

Step 4. Let $h, k \in \mathbb{R}^{d}, \psi \in \mathcal{D}_{N_{0}}, \rho \in \mathbb{R}, t>0, \gamma, \gamma^{*} \in(0,1)$ and $\alpha, \beta$ be multi-indices with $|\alpha|+\gamma \leq N+v$ and $|\beta|+\gamma^{*} \leq N^{*}+v^{*}$. Then

$$
\begin{aligned}
& \left\|L(h) U_{\rho} \partial^{\alpha} T_{1} S_{t} T_{2} \partial^{\beta} U_{-\rho} L(-k)-U_{\rho} \partial^{\alpha} T_{1} S_{t} T_{2} \partial^{\beta} U_{-\rho}\right\|_{1 \rightarrow \infty} \\
& \leq\left\|(I-L(h)) U_{\rho} \partial^{\alpha} T_{1} S_{t / 2} U_{-\rho}\right\|_{2 \rightarrow \infty}\left\|(I-L(k)) U_{\rho} \partial^{\beta} T_{2}^{*} S_{t / 2}^{*} U_{-\rho}\right\|_{2 \rightarrow \infty} \\
& +\left\|(I-L(h)) U_{\rho} \partial^{\alpha} T_{1} S_{t / 2} U_{-\rho}\right\|_{2 \rightarrow \infty}\left\|U_{\rho} \partial^{\beta} T_{2}^{*} S_{t / 2}^{*} U_{-\rho}\right\|_{2 \rightarrow \infty} \\
& +\left\|U_{\rho} \partial^{\alpha} T_{1} S_{t / 2} U_{-\rho}\right\|_{2 \rightarrow \infty}\left\|(I-L(k)) U_{\rho} \partial^{\beta} T_{2}^{*} S_{t / 2}^{*} U_{-\rho}\right\|_{2 \rightarrow \infty} \\
& \leq a_{5}\left(1+\omega_{3}\left(1+\rho^{2}\right) t\right)^{\frac{d+|\alpha|+|\beta|+\gamma+\gamma^{*}}{2}} t^{-\frac{d+|\alpha|+|\beta|}{2}} e^{\omega \rho^{2} t} \\
& \times\left(\left(\frac{|h|}{\sqrt{t}}\right)^{\gamma}\left(\frac{|k|}{\sqrt{t}}\right)^{\gamma^{*}}+\left(\frac{|h|}{\sqrt{t}}\right)^{\gamma}+\left(\frac{|k|}{\sqrt{t}}\right)^{\gamma^{*}}\right),
\end{aligned}
$$

where $a_{5}=2^{(d+|\alpha|+|\beta|+2) / 2} a_{4}^{2}$. Similarly,

$\left\|U_{\rho} \partial^{\alpha} T_{1} S_{t} T_{2} \partial^{\beta} U_{-\rho}\right\|_{1 \rightarrow \infty} \leq a_{5}\left(1+\omega_{3}\left(1+\rho^{2}\right) t\right)^{\frac{d+|\alpha|+|\beta|}{2}} t^{-\frac{d+|\alpha|+|\beta|}{2}} e^{\omega \rho^{2} t}$.

Step 5. Let $t>0$ and $\alpha, \beta$ be multi-indices with $|\alpha| \leq N$ and $|\beta| \leq N^{*}$. Choosing $\rho=0$ it follows from (A.5) and the Dunford-Pettis theorem that the operator $\partial^{\alpha} T_{1} S_{t} T_{2} \partial^{\beta}$ has a kernel $K_{t}^{(\alpha, \beta)} \in L_{\infty}\left(\mathbb{R}^{d} \times \mathbb{R}^{d}\right)$. If $\widetilde{L}$ denotes the $\mathrm{w}^{*}$-continuous left regular representation of $\mathbb{R}^{d} \times \mathbb{R}^{d}$ in $L_{\infty}\left(\mathbb{R}^{d} \times \mathbb{R}^{d}\right)$, then it follows from (A.4) that

$$
\begin{aligned}
& \left\|(I-\tilde{L}(h, k)) K_{t}^{(\alpha, \beta)}\right\|_{\infty} \\
& \leq a_{5} t^{-\frac{d+|\alpha|+|\beta|}{2}}\left(1+\omega_{3} t\right)^{\frac{d+|\alpha|+|\beta|+v+v^{*}}{2}}\left(\left(\frac{|h|}{\sqrt{t}}\right)^{v}\left(\frac{|k|}{\sqrt{t}}\right)^{v^{*}}+\left(\frac{|h|}{\sqrt{t}}\right)^{v}+\left(\frac{|k|}{\sqrt{t}}\right)^{v^{*}}\right)
\end{aligned}
$$

for all $(h, k) \in \mathbb{R}^{d} \times \mathbb{R}^{d}$. So $\lim _{(h, k) \rightarrow(0,0)}\left\|(I-\widetilde{L}(h, k)) K_{t}^{(\alpha, \beta)}\right\|_{\infty}=0$ and $K_{t}^{(\alpha, \beta)}$ is uniformly continuous on $\mathbb{R}^{d} \times \mathbb{R}^{d}$. 
Define $K_{t}=K_{t}^{(\alpha, \beta)}$ if $|\alpha|=|\beta|=0$. Thus $K_{t}$ is the kernel of $T_{1} S_{t} T_{2}$. Let $|\alpha| \leq N,|\beta| \leq N^{*}$ and $t>0$. Then for all $u, v \in C_{c}^{\infty}\left(\mathbb{R}^{d}\right)$ one has

$$
\begin{aligned}
(-1)^{|\alpha|+|\beta|} \int_{\mathbb{R}^{d}} \int_{\mathbb{R}^{d}} K_{t}(x, y)\left(\partial^{\alpha} u\right)(x)\left(\partial^{\beta} v\right)(y) d x d y \\
=(-1)^{|\alpha|+|\beta|}\left(T_{1} S_{t} T_{2} \partial^{\beta} v, \partial^{\alpha} \bar{u}\right) \\
=(-1)^{|\beta|}\left(\partial^{\alpha} T_{1} S_{t} T_{2} \partial^{\beta} v, \bar{u}\right) \\
=(-1)^{|\beta|} \int_{\mathbb{R}^{d}} u(x)\left(\partial^{\alpha} T_{1} S_{t} T_{2} \partial^{\beta} v\right)(x) d x \\
=(-1)^{|\beta|} \int_{\mathbb{R}^{d}} \int_{\mathbb{R}^{d}} K_{t}^{(\alpha, \beta)}(x, y) u(x) v(y) d x d y .
\end{aligned}
$$

So by density

$$
\begin{array}{r}
(-1)^{|\alpha|+|\beta|} \int_{\mathbb{R}^{d} \times \mathbb{R}^{d}} K_{t}(x, y)\left(\partial_{x}^{\alpha} \partial_{y}^{\beta} w\right)(x, y) d(x, y) \\
=(-1)^{|\beta|} \int_{\mathbb{R}^{d} \times \mathbb{R}^{d}} K_{t}^{(\alpha, \beta)}(x, y) w(x, y) d(x, y)
\end{array}
$$

for all $w \in C_{c}^{\infty}\left(\mathbb{R}^{d} \times \mathbb{R}^{d}\right)$ and the $(-1)^{|\beta|} K_{t}^{(\alpha, \beta)}$ are the successive distributional derivatives of $K_{t}$. Since the $K_{t}^{(\alpha, \beta)}$ are continuous one deduces from the lemma of Du Bois-Reymond that $K_{t}$ is $N$ times differentiable in the first variable, the derivatives are $N^{*}$-times differentiable in the second variable and all derivatives are continuous.

Step 6. Let $|\alpha| \leq N,|\beta| \leq N^{*}, t>0$ and $x, y \in \mathbb{R}^{d}$. Then it follows from (A.5) that

$$
\left|K_{t}^{(\alpha, \beta)}(x, y)\right| \leq a_{5}\left(1+\omega_{3}\left(1+\rho^{2}\right) t\right)^{\frac{d+|\alpha|+|\beta|}{2}} t^{-\frac{d+|\alpha|+|\beta|}{2}} e^{\omega \rho^{2} t} e^{-\rho(\psi(x)-\psi(y))}
$$

for all $\rho \geq 0$ and $\psi \in \mathcal{D}_{N_{0}}$. Minimizing over $\psi$ and using (A.1) gives

$$
\left|K_{t}^{(\alpha, \beta)}(x, y)\right| \leq a_{5}\left(1+\omega_{3}\left(1+\rho^{2}\right) t\right)^{\frac{d+|\alpha|+|\beta|}{2}} t^{-\frac{d+|\alpha|+|\beta|}{2}} e^{\omega \rho^{2} t} e^{-\rho c|x-y|}
$$

and with the choice $\rho=\frac{c|x-y|}{2 \omega t}$ one deduces that

$$
\left|K_{t}^{(\alpha, \beta)}(x, y)\right| \leq a_{5}\left(1+\omega_{3}\left(t+\frac{c^{2}|x-y|^{2}}{4 \omega^{2} t}\right)\right)^{\frac{d+|\alpha|+|\beta|}{2}} t^{-\frac{d+|\alpha|+|\beta|}{2}} e^{-b \frac{|x-y|^{2}}{t}} .
$$

This proves the bounds (A.2). 
Step 7. Let $\alpha, \beta$ be multi-indices, $\gamma, \gamma^{*} \in(0,1)$ and suppose that $|\alpha|+|\gamma| \leq N+v$ and $|\beta|+\gamma^{*} \leq N^{*}+\nu^{*}$. Let $\kappa>0$ and $\tau \in[0,1)$. There exists a $\tau_{1} \in(\tau, 1)$ such that

$$
b_{1}=b\left(1-\tau_{1}\right)^{2} \frac{c}{c+2 \tau} .
$$

Set $\lambda=\frac{b}{b_{1}} \geq 1$. Further, let $\varepsilon, \eta \in(0,1)$. Let $x, y, h, k \in \mathbb{R}^{d}, t>0$ and suppose that $|h|+|k| \leq \kappa \sqrt{t}+\tau|x-y|$. Let $\rho \in \mathbb{R}$ and $\psi \in \mathcal{D}_{N_{0}}$.

If $R H S=a_{5} \ldots$ denotes the right hand side of (A.4), then it follows from (A.4) that

$$
\left|e^{-\rho \psi(x)} K_{t}^{(\alpha, \beta)}(x, y) e^{\rho \psi(y)}-e^{-\rho \psi(x-h)} K_{t}^{(\alpha, \beta)}(x-h, y-k) e^{\rho \psi(y-k)}\right| \leq R H S .
$$

So

$$
\begin{aligned}
& \left|K_{t}^{(\alpha, \beta)}(x, y)-e^{\rho(\psi(x)-\psi(x-h))} K_{t}^{(\alpha, \beta)}(x-h, y-k) e^{-\rho(\psi(y)-\psi(y-k))}\right| \\
& \quad \leq R H S \cdot e^{\rho(\psi(x)-\psi(y))}
\end{aligned}
$$

and

$$
\begin{aligned}
& \left|K_{t}^{(\alpha, \beta)}(x, y)-K_{t}^{(\alpha, \beta)}(x-h, y-k)\right| \\
\leq & R H S \cdot e^{\rho(\psi(x)-\psi(y))} \\
& +\left|1-e^{\rho(\psi(x)-\psi(x-h))} e^{-\rho(\psi(y)-\psi(y-k))}\right|\left|K_{t}^{(\alpha, \beta)}(x-h, y-k)\right| \\
\leq & R H S \cdot e^{\rho(\psi(x)-\psi(y))}+|\rho|(|h|+|k|) e^{|\rho|(|h|+|k|)}\left|K_{t}^{(\alpha, \beta)}(x-h, y-k)\right| .
\end{aligned}
$$

Suppose $\rho \geq 0$. Optimizing over $\psi$ gives

$$
\begin{aligned}
& \left|K_{t}^{(\alpha, \beta)}(x, y)-K_{t}^{(\alpha, \beta)}(x-h, y-k)\right| \\
& \leq R H S \cdot e^{-c \rho|x-y|}+\rho(|h|+|k|) e^{\rho(|h|+|k|)}\left|K_{t}^{(\alpha, \beta)}(x-h, y-k)\right| \\
& \leq a_{5}\left(1+\omega_{3}\left(1+\rho^{2}\right) t\right)^{E} t^{-\widetilde{E}} e^{\omega \rho^{2} t} e^{-c \rho|x-y|} \\
& \quad \times\left(\left(\frac{|h|}{\sqrt{t}}\right)^{\gamma}\left(\frac{|k|}{\sqrt{t}}\right)^{\gamma^{*}}+\left(\frac{|h|}{\sqrt{t}}\right)^{\gamma}+\left(\frac{|k|}{\sqrt{t}}\right)^{\gamma^{*}}\right) \\
& \quad+\rho(|h|+|k|) e^{\rho(|h|+|k|)}\left|K_{t}^{(\alpha, \beta)}(x-h, y-k)\right|,
\end{aligned}
$$

where for brevity we set $E=\frac{d+|\alpha|+|\beta|+\gamma+\gamma^{*}}{2}$ and $\widetilde{E}=\frac{d+|\alpha|+|\beta|}{2}$. Choose $\rho=$ $\frac{c|x-y|}{2 \lambda \omega t}$. Before we estimate both terms in (A.7) we need one more estimate to replace the denominator $\sqrt{t}$ by $\sqrt{t}+|x-y|$. 
Since $\frac{|x-y|}{\sqrt{t}} \leq \frac{1}{\sqrt{\varepsilon}} e^{\varepsilon \frac{|x-y|^{2}}{t}}$ it follows that

$$
t^{-1 / 2}(\sqrt{t}+|x-y|)=1+\frac{|x-y|}{\sqrt{t}} \leq \frac{2}{\sqrt{\varepsilon}} e^{\varepsilon \frac{|x-y|^{2}}{t}}
$$

and

$$
t^{-1 / 2} \leq \frac{2}{\sqrt{\varepsilon}} \frac{1}{\sqrt{t}+|x-y|} e^{\varepsilon \frac{|x-y|^{2}}{t}}
$$

Therefore

$$
\left(\frac{|h|}{\sqrt{t}}\right)^{\gamma}+\left(\frac{|k|}{\sqrt{t}}\right)^{\gamma^{*}} \leq \frac{2}{\sqrt{\varepsilon}}\left(\left(\frac{|h|}{\sqrt{t}+|x-y|}\right)^{\gamma}+\left(\frac{|k|}{\sqrt{t}+|x-y|}\right)^{\gamma^{*}}\right) e^{\varepsilon \frac{|x-y|^{2}}{t}}
$$

and

$$
\begin{aligned}
\left(\frac{|h|}{\sqrt{t}}\right)^{\gamma}\left(\frac{|k|}{\sqrt{t}}\right)^{\gamma^{*}} & \leq \frac{4}{\varepsilon}\left(\frac{|h|}{\sqrt{t}+|x-y|}\right)^{\gamma}\left(\frac{|k|}{\sqrt{t}+|x-y|}\right)^{\gamma^{*}} e^{2 \varepsilon \frac{|x-y|^{2}}{t}} \\
& \leq \frac{4}{\varepsilon}(\kappa+\tau)^{\gamma^{*}}\left(\frac{|h|}{\sqrt{t}+|x-y|}\right)^{\gamma} e^{2 \varepsilon \frac{|x-y|^{2}}{t}} .
\end{aligned}
$$

We estimate both terms in (A.7) separately.

For the first term note that

$$
\omega \rho^{2} t-c \rho|x-y|=-\frac{c^{2}|x-y|^{2}}{4 \omega t} \frac{2 \lambda-1}{\lambda^{2}} \leq-\frac{b}{\lambda} \frac{|x-y|^{2}}{t}=-b_{1} \frac{|x-y|^{2}}{t} .
$$

Therefore the first term in (A.7) can be estimated by

$$
\begin{aligned}
\frac{6}{\varepsilon} a_{5}(1+\kappa)\left(1+\omega_{3} t+\frac{\omega_{3} c^{2}|x-y|^{2}}{4 \lambda^{2} \omega^{2} t}\right)^{E} t^{-\widetilde{E}}\left(\left(\frac{|h|}{\sqrt{t}+|x-y|}\right)^{\gamma}\right. \\
\left.+\left(\frac{|k|}{\sqrt{t}+|x-y|}\right)^{\gamma^{*}}\right) e^{-\left(b_{1}-2 \varepsilon\right) \frac{|x-y|^{2}}{t}}
\end{aligned}
$$

For the second term we use (A.6) to estimate

$$
\left|K_{t}^{(\alpha, \beta)}(x-h, y-k)\right| \leq a_{5}\left(1+\omega_{3}\left(t+\frac{c^{2}|x-y-h+k|^{2}}{4 \omega^{2} t}\right)\right)^{\widetilde{E}} t^{-\widetilde{E}} e^{-b \frac{|x-y-h+k|^{2}}{t}} .
$$

Clearly

$$
|x-y-h+k|^{2} \leq 2|x-y|^{2}+2(|h|+|k|)^{2} \leq 6|x-y|^{2}+4 \kappa^{2} t .
$$


For the exponential set $\eta=\frac{\tau_{1}}{1-\tau_{1}}>0$ and $\delta=\frac{\tau_{1}^{2}}{\tau^{2}}-1>0$. Then

$$
\begin{aligned}
-b \frac{|x-y-h+k|^{2}}{t} & \leq-b \frac{|x-y|^{2}}{(1+\eta) t}+b \frac{|h-k|^{2}}{\eta t} \\
& \leq-b \frac{|x-y|^{2}}{(1+\eta) t}+\frac{b}{\eta t}\left((1+\delta) \tau^{2}|x-y|^{2}+\left(1+\delta^{-1}\right) \kappa^{2} t\right) \\
& =-b\left(1-\tau_{1}\right)^{2} \frac{|x-y|^{2}}{t}+b \kappa^{2} \frac{\tau_{1}-\tau_{1}^{2}}{\tau_{1}^{2}-\tau^{2}} .
\end{aligned}
$$

So

$$
\begin{aligned}
& \left|K_{t}^{(\alpha, \beta)}(x-h, y-k)\right| \\
& \leq a_{5}\left(1+\frac{\omega_{3} c^{2} \kappa^{2}}{\omega^{2}}+\omega_{3} t+\frac{3 \omega_{3} c^{2}|x-y|^{2}}{2 \omega^{2} t}\right)^{\widetilde{E}} t^{-\widetilde{E}} e^{-b\left(1-\tau_{1}\right)^{2} \frac{|x-y|^{2}}{t}} \exp \left(b \kappa^{2} \frac{\tau_{1}-\tau_{1}^{2}}{\tau_{1}^{2}-\tau^{2}}\right) .
\end{aligned}
$$

Next we estimate the factor $\rho(|h|+|k|) e^{\rho(|h|+|k|)}$. One has

$$
\rho(|h|+|k|) \leq \frac{c|x-y|}{2 \lambda \omega t}(\kappa \sqrt{t}+\tau|x-y|) \leq \frac{c(\tau+\eta \kappa)}{2 \lambda \omega} \frac{|x-y|^{2}}{t}+\frac{c \kappa}{2 \eta \lambda \omega} .
$$

and alternatively

$$
\begin{aligned}
\rho(|h|+|k|) & \leq \frac{c|x-y|}{2 \lambda \omega \sqrt{t}} \frac{|h|+|k|}{\sqrt{t}} \\
& \leq \frac{c|x-y|}{\sqrt{\varepsilon} \lambda \omega \sqrt{t}} \frac{|h|+|k|}{\sqrt{t}+|x-y|} e^{\varepsilon \frac{|x-y|^{2}}{t}} \\
& \leq \frac{c(1+\kappa)^{2}}{\varepsilon \lambda \omega} e^{2 \varepsilon \frac{|x-y|^{2}}{t}}\left(\left(\frac{|h|}{\sqrt{t}+|x-y|}\right)^{\gamma}+\left(\frac{|k|}{\sqrt{t}+|x-y|}\right)^{\gamma^{*}}\right) .
\end{aligned}
$$

So

$$
\begin{aligned}
\rho(|h|+|k|) e^{\rho(|h|+|k|)} \leq & \frac{c(1+\kappa)^{2}}{\varepsilon \lambda \omega} \exp \left(\frac{c \kappa}{2 \eta \lambda \omega}\right) \exp \left(\left(\frac{c(\tau+\eta \kappa)}{2 \lambda \omega}+2 \varepsilon\right) \frac{|x-y|^{2}}{t}\right) \\
& \times\left(\left(\frac{|h|}{\sqrt{t}+|x-y|}\right)^{\gamma}+\left(\frac{|k|}{\sqrt{t}+|x-y|}\right)^{\gamma^{*}}\right) .
\end{aligned}
$$


Using the identity $b\left(1-\tau_{1}\right)^{2}-\frac{c \tau}{2 \lambda \omega}=b_{1}$ one deduces that the second term in (A.7) can be estimated by

$$
\begin{gathered}
\frac{a_{5} c(1+\kappa)^{2}}{\varepsilon \lambda \omega} \exp \left(\frac{c \kappa}{2 \eta \lambda \omega}\right) \exp \left(b \kappa^{2} \frac{\tau_{1}-\tau_{1}^{2}}{\tau_{1}^{2}-\tau^{2}}\right)\left(1+\frac{\omega_{3} c^{2} \kappa^{2}}{\omega^{2}}+\omega_{3} t+\frac{3 \omega_{3} c^{2}|x-y|^{2}}{2 \omega^{2} t}\right)^{\widetilde{E}} \\
\times t^{-\widetilde{E}}\left(\left(\frac{|h|}{\sqrt{t}+|x-y|}\right)^{\gamma}+\left(\frac{|k|}{\sqrt{t}+|x-y|}\right)^{\gamma^{*}}\right) e^{-\left(b_{1}-2 \varepsilon-\frac{c \kappa \eta}{2 \lambda \omega}\right) \frac{|x-y|^{2}}{t}}
\end{gathered}
$$

Then (A.3) follows.

\section{References}

[1] D. Albrecht, X. Duong and A. MCIntosh, Operator theory and harmonic analysis, In: "Instructional Workshop on Analysis and Geometry, Part III", Proceedings of the Centre for Mathematics and its Applications. Australian National University, Canberra 34 (1996), 77-136.

[2] W. ARENDT and A. V. BukhVAlov, Integral representations of resolvents and semigroups, Forum Math. 6 (1994), 111-135.

[3] W. ARENDT and A. F. TER Elst, Sectorial forms and degenerate differential operators, J. Operator Theory 67 (2012), 33-72.

[4] P. Auscher, S. Hofmann, M. Lacey, A. Mcintosh and P. Tchamitchian, The solution of the Kato square root problem for second order elliptic operators on $\mathbb{R}^{n}$, Ann. of Math. (2) 156 (2002), 633-654.

[5] A. F. M. TER Elst and E. M. Ouhabaz, Partial Gaussian bounds for degenerate differential operators, Potential Anal. 35 (2011), 175-199.

[6] A. F. M. Ter Elst and E. M. OuhabaZ, Partial spectral multipliers and partial Riesz. transforms for degenerate operators, Rev. Mat. Iberoamericana 29 (2012), 691-713.

[7] A. F. M. TER ElST and D. W. RobINSON, On Kato's square root problem, Hokkaido Math. J. 26 (1997), 365-376.

[8] A. F. M. TER ELST and D. W. RobInSON, Second-order strongly elliptic operators on Lie groups with Hölder continuous coefficients, J. Austr. Math. Soc. (Series A) 63 (1997), 297-363.

[9] A. F. M. TER ElST and D. W. RobINSON, Second-order subelliptic operators on Lie groups I: complex uniformly continuous principal coefficients, Acta Appl. Math. 59 (1999), 299-331.

[10] A. F. M. TER Elst and D. W. Robinson, Second-order subelliptic operators on Lie groups III: Hölder continuous coefficients, Calc. Var. Partial Differential Equations 8 (1999), 327-363.

[11] M. Giaquinta, "Multiple Integrals in the Calculus of Variations and Nonlinear Elliptic Systems", Annals of Mathematics Studies 105, Princeton University Press, Princeton, 1983.

[12] M. GIAQUiNTA and L. MARTINAZZI, "An Introduction to the Regularity Theory for Elliptic Systems, Harmonic Maps and Minimal Graphs", Edizioni della Normale, Pisa, 2005.

[13] T. KATO, Fractional powers of dissipative operators, J. Math. Soc. Japan 13 (1961), 246274.

[14] T. Kato, Fractional powers of dissipative operators, II, J. Math. Soc. Japan 14 (1962), 242-248. 
[15] T. Kaто, "Perturbation Theory for Linear Operators", Second edition, Grundlehren der mathematischen Wissenschaften 132. Springer-Verlag, Berlin etc., 1980.

[16] A. McIntosh, Square roots of elliptic operators, J. Funct. Anal. 61 (1985), 307-327.

[17] E. M. OuhabAz, "Analysis of Heat Equations on Domains", Vol. 31 of London Mathematical Society Monographs Series, Princeton University Press, Princeton, NJ, 2005.

[18] E. M. OuhabAZ, Comportement des noyaux de la chaleur des opérateurs de Schrödinger et applications à certaines équations paraboliques semi-linéaires, J. Funct. Anal. 238 (2006), 278-297.

[19] B. SimON, A canonical decomposition for quadratic forms with applications to monotone convergence theorems, J. Funct. Anal. 28 (1978), 377-385.

[20] E. M. STEIN, "Harmonic Analysis: Real-variable Methods, Orthogonality, and Oscillatory Integrals", Princeton University Press, Princeton, 1993.

[21] C. J. XU and C. ZUILY, Higher interior regularity for quasilinear subelliptic operators, Calc. Var. Partial Differential Equations 5 (1997), 323-343.

Department of Mathematics

University of Auckland

Private bag 92019

Auckland 1142, New Zealand

Institut de Mathématiques de Bordeaux

Univ. Bordeaux, UMR 5251

351, Cours de la Libération

33405 Talence, France 